\title{
One-loop correlators and BCJ numerators from forward limits
}

\author{
Alex Edison, ${ }^{a}$ Song He, ${ }^{b, c, d, e}$ Oliver Schlotterer $^{a}$ and Fei Teng ${ }^{a}$ \\ ${ }^{a}$ Department of Physics and Astronomy, Uppsala University, \\ SE-75108 Uppsala, Sweden \\ ${ }^{b}$ CAS Key Laboratory of Theoretical Physics, Institute of Theoretical Physics, \\ Chinese Academy of Sciences, Beijing 100190, China \\ ${ }^{c}$ School of Physical Sciences, University of Chinese Academy of Sciences, \\ No.19A Yuquan Road, Beijing 100049, China \\ ${ }^{d}$ School of Fundamental Physics and Mathematical Sciences, \\ Hangzhou Institute for Advanced Study, UCAS, Hangzhou 310024, China \\ e International Centre for Theoretical Physics Asia-Pacific, \\ Beijing/Hangzhou, China \\ E-mail: alexander.edison@physics.uu.se, songhe@itp.ac.cn, \\ oliver.schlotterer@physics.uu.se, fei.teng@physics.uu.se
}

ABSTRACT: We present new formulas for one-loop ambitwistor-string correlators for gauge theories in any even dimension with arbitrary combinations of gauge bosons, fermions and scalars running in the loop. Our results are driven by new all-multiplicity expressions for tree-level two-fermion correlators in the RNS formalism that closely resemble the purely bosonic ones. After taking forward limits of tree-level correlators with an additional pair of fermions/bosons, one-loop correlators become combinations of Lorentz traces in vector and spinor representations. Identities between these two types of traces manifest all supersymmetry cancellations and the power counting of loop momentum. We also obtain parity-odd contributions from forward limits with chiral fermions. One-loop numerators satisfying the Bern-Carrasco-Johansson (BCJ) duality for diagrams with linearized propagators can be extracted from such correlators using the well-established tree-level techniques in YangMills theory coupled to biadjoint scalars. Finally, we obtain streamlined expressions for BCJ numerators up to seven points using multiparticle fields.

Keywords: Scattering Amplitudes, Superstrings and Heterotic Strings, Supersymmetric Gauge Theory

ARXIV EPRINT: 2005.03639 


\section{Contents}

1 Introduction 1

1.1 Conventions 3

1.2 Summary 4

2 Basics 5

2.1 Vertex operators 5

2.2 Tree-level correlator for external bosons 6

$\begin{array}{lll}2.3 & \text { Tree-level correlator for two external fermions } & 7\end{array}$

2.4 Alternative representation of the two-fermion correlator 8

$\begin{array}{lll}2.5 & \text { Forward limits and gluing operators } & 10\end{array}$

3 One loop correlators and numerators of ten-dimensional SYM $\quad 10$

$\begin{array}{ll}3.1 \text { The forward limit of two bosons/fermions } & 11\end{array}$

$\begin{array}{lll}3.2 & \text { From spinor traces to vector ones } & 12\end{array}$

$\begin{array}{lll}3.3 & \text { Ten-dimensional SYM } & 14\end{array}$

3.4 BCJ numerators versus single-trace $\mathrm{YM}+\phi^{3}$ at tree level $\quad 16$

4 General gauge theories $\quad 18$

$\begin{array}{ll}4.1 & \text { Forward limits in general gauge theories } \\ & 18\end{array}$

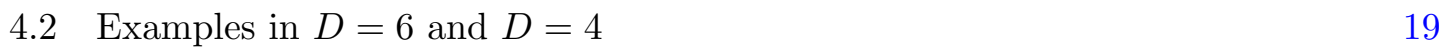

5 Parity-odd contributions $\quad 21$

5.1 General prescription and low-multiplicity validation 22

5.2 Anomalies and their singled-out leg 24

6 BCJ numerators in terms of multiparticle fields $\quad 25$

$\begin{array}{ll}6.1 \text { Brief review } & 26\end{array}$

$\begin{array}{ll}6.2 D=10 \text { examples } & 27\end{array}$

$\begin{array}{lll}6.3 D & =6 \text { examples } & 29\end{array}$

6.4 Parity-odd examples $\quad 32$

$\begin{array}{lll}7 & \text { Summary and outlook } & 32\end{array}$

A One-loop integrands with linear propagators and CHY formulas 33

B Conformal field theory and tree-level correlators $\quad 35$

$\begin{array}{lll}\text { B.1 CFT basics } & 35\end{array}$

B.2 Bosonic correlators and the Pfaffian 36

B.3 Two-fermion correlators 37

$\begin{array}{lll}\text { B.4 Four-fermion correlators } & 39\end{array}$ 
C.1 Decomposition of $\operatorname{tr}_{\mathrm{S}} \rightarrow \operatorname{tr}_{\mathrm{V}} \quad 40$

C.2 Higher $t_{2 n}$ tensors from $D=10 \mathrm{SYM} \quad 42$

C.3 Parity-odd traces 43

\section{Introduction}

Recent years have witnessed enormous progress in understanding novel structures and symmetries of scattering amplitudes in various theories, as well as surprising connections between them. One important example is the Bern-Carrasco-Johansson (BCJ) duality between color and kinematics in gauge theories, and double-copy relations to corresponding gravity theories [1-3], see [4] for a review.

The color-kinematic duality states that in a trivalent-diagram expansion of gaugetheory amplitudes, kinematic factors can be arranged to satisfy the same algebraic relations as color factors. Kinematic factors with this property are known as BCJ numerators. Based on this, a remarkable conjecture is that loop integrands for gravity amplitudes can be obtained from gauge-theory ones by simply substituting color factors for another copy of such BCJ numerators. At tree level, the double copy is equivalent to the field-theory limit of the famous Kawai-Lewellen-Tye (KLT) relations between open- and closed-string amplitudes [5], and the BCJ duality has also been proven directly [6]. In the quantum regime, this double-copy construction has led to great advances in the study of the ultraviolet behavior of supergravity amplitudes [7-12]. However, it remains a conjecture and the principle behind it is poorly understood.

Apart from the original KLT relations, string theory has provided constructions of BCJ numerators at tree and loop level [13-17]. ${ }^{1}$ Relatedly, worldsheet methods originating from the Cachazo-He-Yuan (CHY) formulation [25, 26] have been a major driving force in understanding and extending BCJ duality and the double copy. Based on scattering equations [27], CHY formulas express tree amplitudes in a large class of massless theories as worldsheet integrals which can often be derived from ambitwistor string theories [2832]. These methods have not only led to new double-copy realizations and connections for various theories [33-35], using loop-level CHY/ambitwistor strings [30, 36-42], they have also extended KLT and BCJ double copy to one-loop level [43, 44]. Based on nodal Riemann spheres, loop-level CHY/ambitwistor-string formulas yield loop amplitudes in a new representation of their Feynman integrals with propagators linear in loop momenta; alternatively they can be understood as forward limits of tree amplitudes with a pair of momenta in higher dimensions [38, 45].

\footnotetext{
${ }^{1}$ Similarly, the gauge invariant reformulation of the color-kinematics duality via BCJ relations can be elegantly derived from monodromy properties of open-string worldsheets [18, 19]. See [20-24] for loop-level extensions of monodromy relations among string amplitudes.
} 
In this paper, we continue the study of the loop-level BCJ duality and double copy based on worldsheet methods. In particular, we present new results on all-multiplicity oneloop BCJ numerators for Feynman integrals with propagators linear in loop momentum, which extends and offers a new perspective on the algorithm of [44]. Starting from the worldsheet correlator with external gluons, one can obtain $n$-gon master numerators by extracting the coefficient of Parke-Taylor factors with all possible orderings. As reviewed in appendix A, BCJ representations of one-loop integrands with linearized propagators arise naturally from one-loop CHY formulas [36, 37],,$^{2}$ also see [49-53] for the relation between linearized and quadratic propagators.

In the RNS formulation of the ambitwistor string [28, 30], the correlator takes the form of a one-loop Pfaffian, where the amount of spacetime supersymmetry is reflected by the relative weights of different spin structures ${ }^{3}$ [37]. The key of the algorithm in [44] is to reduce the dependence on worldsheet punctures to Parke-Taylor factors via repeated use of one-loop scattering equations, which can be rather tedious in practice. It is thus highly desirable to tame this technical difficulty by using a representation of the one-loop correlator that is more suitable for extracting BCJ numerators. This is one of the major achievements of the current paper.

The simplest one-loop correlators arise when the states of $D=10$ super Yang-Mills (SYM) circulate in the loop. As we will review shortly, these one-loop correlators receive contributions from forward limits of tree-level correlators with an additional pair of bosons (gluons) and those with fermions (gluinos). ${ }^{4}$ Since tree-level correlators of bosons can be simplified to the well-known Pfaffian [25], it is highly desirable to also bring the two-fermion correlators into Pfaffian form in order to control the supersymmetry cancellations between their forward limits. For this purpose we will derive a new representation of the two-fermion correlator tailored to expose its interplay with the bosonic correlator under forward limits. This representation realizes the gluing-operator prescription of Roehrig and Skinner [65].

Similarly we will derive one-loop correlators for general gauge theories in even dimension $D<10$ via forward limits in an arbitrary combination of scalars, fermions and gauge bosons in the loop. ${ }^{5}$ The main advantage of our new representations of fermionic corre-

\footnotetext{
${ }^{2}$ See $[46,47]$ for an alternative approach to one-loop CHY formulas based on the $\Lambda$ scattering equations [48].

${ }^{3}$ Spin structures refer to the boundary conditions of the worldsheet fermions in the RNS formalism as they are taken around the two homology cycles of the worldsheet torus. The contributions of individual spin structures to the one-loop correlators are weighted by partition functions that reflect the amount of spacetime supersymmetry. The interplay between different spin structures in multiparticle correlators has been studied in the context of conventional strings [54-58] and ambitwistor strings [44].

${ }^{4}$ We remark that tree-level correlators and BCJ numerators for any combination of external bosons and fermions can be extracted from their representation in pure-spinor superspace [13, 14, 59], see [29, 31] for a pure-spinor incarnation of the ambitwistor string. Even though the extraction of components can be obtained for any number of legs $[60,61]$, these are not the correlator representations that we will use in the forward-limit analysis of this work. One-loop correlators in pure-spinor superspace up to and including seven external legs can be found in [62-64].

${ }^{5}$ The algorithm for one-loop BCJ numerators in [44] has been formulated for gauge theories with at least four supercharges, and non-supersymmetric four-point BCJ numerators have been derived from forward limits in [40]. The method here certainly applies to the non-supersymmetric case in absence of fermion correlators.
} 
lators is that the extraction of BCJ numerators becomes a problem that has been solved at tree level: the dependence on worldsheet punctures of one-loop correlators turns out to be identical to that of tree correlators for single-trace amplitudes in Yang-Mills coupled to biadjoint scalars $\left(\mathrm{YM}+\phi^{3}\right)$ [33]. The reduction of the latter to Parke-Taylor factors (or equivalently extracting BCJ numerators for such amplitudes $[35,66]$ ) has been studied extensively [67-72], and we can directly apply these results to our one-loop problem.

As a result, we will present new expressions for BCJ numerators, not only for tendimensional SYM but also for lower-dimensional gauge theories with reduced or without spacetime supersymmetry. The numerators of this work manifest the power counting of loop momenta by representation-theoretic identities between Lorentz traces in vector and spinor representations. Moreover, our construction preserves locality, i.e.the BCJ numerators do not involve any poles in momentum invariants.

Furthermore, we will also present two new results on one-loop correlators and BCJ numerators. First, we will compute parity-odd contributions to the correlators by taking forward limits with chiral fermions, both in $D=10 \mathrm{SYM}$ and in the $D=6$ case with a chiral spectrum. In addition, we will simplify the BCJ numerators using the so-called multiparticle fields [61, 73], which can be viewed as numerators of Berends-Giele currents [74] that respect color-kinematics duality, derived in the BCJ gauge [60, 75].

\subsection{Conventions}

In the conventions of this paper, the CHY representation of tree-level amplitudes with a double-copy structure is given by

$$
\mathcal{M}_{L \otimes R}^{\text {tree }}=\mathscr{N} \int \mathrm{d} \mu_{n}^{\text {tree }} \mathcal{I}_{L}^{\text {tree }} \mathcal{I}_{R}^{\text {tree }}, \quad \mathrm{d} \mu_{n}^{\text {tree }} \equiv \frac{\mathrm{d}^{n} \sigma}{\operatorname{Vol}[\operatorname{SL}(2, \mathbb{C})]} \prod_{i=1}^{n} \delta\left(E_{i}\right),
$$

where the theory-dependent normalization factor $\mathscr{N}$ for instance specializes to $-2\left(-\frac{g}{\sqrt{2}}\right)^{n-2}$ for gauge-theory amplitudes with YM coupling $g .{ }^{6}$ Inside the CHY measure $\mathrm{d} \mu_{n}^{\text {tree }}$, the prime along with the product $\prod^{\prime}$ instructs to only impose the $n-3$ independent scattering equations for the punctures $\sigma_{j} \in \mathbb{C}$ on the Riemann sphere,

$$
E_{i} \equiv \sum_{\substack{j=1 \\ j \neq i}}^{n} \frac{k_{i} \cdot k_{j}}{\sigma_{i j}}=0, \quad \sigma_{i j} \equiv \sigma_{i}-\sigma_{j}
$$

see [72] for additional details. Depending on the choice of the half-integrands $\mathcal{I}_{L, R}^{\text {tree }},(1.1)$ can be specialized to yield tree amplitudes in gauge theories, (super-)gravity and a variety of further theories $[33,76]$. Color-ordered gauge-theory amplitudes are obtained from a Parke-Taylor factor $\mathcal{I}_{L}^{\text {tree }} \rightarrow\left(\sigma_{12} \sigma_{23} \ldots \sigma_{n 1}\right)^{-1}$ and taking $\mathcal{I}_{R}^{\text {tree }}$ to be the reduced Pfaffian given in (2.5). The one-loop analogue of the amplitude prescription (1.1) is reviewed in appendix A.

\footnotetext{
${ }^{6}$ The combination $g / \sqrt{2}$ in the normalization factor $\mathscr{N}$ of gauge-theory amplitudes can be understood as rescaling the color factors.
} 


\subsection{Summary}

The main results of the paper can be summarized as follows.

- We present new expressions for tree-level correlators with two and four fermions and any number of bosons. By taking forward limits in a pair of bosons/fermions, we obtain a new formula (3.15) for one-loop correlators in $D=10$ SYM.

- By combining building blocks with vector bosons, fermions or scalars circulating the loop, we obtain a similar formula (4.4) for one-loop correlators in general, possibly non-supersymmetric gauge theories in $D<10$.

- Since the worldsheet dependence is identical to that of single-trace correlators for $\left(\mathrm{YM}+\phi^{3}\right)$ tree amplitudes, we can recycle tree-level results to extract one-loop BCJ numerators in these theories.

- We will derive parity-odd contributions (5.3) to one-loop correlators from forward limits with chiral fermions.

- We present various BCJ numerators at $n \leq 7$ points in a compact form by using the multiparticle fields.

The paper is organized as follows. We start in section 2 by collecting some results which will be used in the subsequent: first we spell out tree-level correlators with $n$ bosons and those with $n-2$ bosons and 2 fermions in the RNS formalism for ambitwistor string theory. Then we review how the tree-level input can be used to construct one-loop correlators by taking the forward limit in a pair of bosons or fermions with momenta in higher dimensions.

Next, we study one-loop correlators and BCJ numerators in $D=10$ SYM in section 3 and express them as combinations of vector traces and spinor traces of linearized field strengths with accompanying Pfaffians. We then propose a key formula (3.11) for converting spinor traces to vector traces, which allows us to simplify the one-loop correlators of $D=10 \mathrm{SYM}$. In particular, the power counting in loop momentum follows from representation-theoretic identities between vector and spinor traces. Once the correlator is written in this form, it is straightforward produce BCJ numerators as the problem is equivalent to that for tree-level amplitudes in $\mathrm{YM}+\phi^{3}$.

We move to general gauge theories in even dimensions $D<10$ in section 4 . By also including one-loop correlators from forward limits in two scalars, we obtain a general formula for the case with $\mathbf{n}_{\mathrm{v}}$ vectors, $\mathbf{n}_{\mathrm{f}}$ Weyl fermions and $\mathbf{n}_{\mathrm{s}}$ scalars. In particular, we apply the general formula to obtain explicit results for specific theories in $D=6$ and $D=4$.

In section 5, we derive parity-odd contributions to one-loop correlators from forward limits in chiral fermions, which are parity-odd completions of correlators in $D=10 \mathrm{SYM}$ and those in lower dimensions. Finally, in section 6 , by using multiparticle fields, we provide particularly compact expressions for the BCJ numerators in various theories, which combine contributions from the Pfaffians and the field-strength traces in the correlators.

The discussion in the main text is complemented by three appendices: our representation of one-loop integrands will be reviewed in appendix A; we review CFT basics and give 
the derivation for tree-level correlators with zero, two and four fermions in appendix B; we also prove the identity for reducing spinor traces to vector traces in appendix C.1.

\section{Basics}

In this section, we use the RNS formulation of the ambitwistor string in $D=10$ dimensions [28, 30] (see [77-79] for the RNS superstring) to review tree-level correlators with $n$ gluons (bosons). The latter evaluate to the well-known Pfaffian in the CHY formulation [25], and we will present new representations for correlators with 2 gluinos (fermions) and $n-2$ gluons, also see appendix B.4 for four-fermion correlators. On the support of scattering equations, the Pfaffian can be expanded into smaller ones dressed by Lorentz contractions of field strengths with two polarizations. As we will see, the correlator with 2 gluinos and $n-2$ gluons can be simplified to a similar form, which features smaller Pfaffians dressed by gamma-matrix contracted field strengths, with wave functions for the two fermions. We will see that these representations of correlators are most suitable for combining the forward limits in two gluons/gluinos and studying the resulting supersymmetry cancellations.

\subsection{Vertex operators}

Let us first review the underlying vertex operators for the gluon with momentum $k_{\mu}$ and polarization vector $\epsilon^{\mu}$ with $\mu=0,1, \ldots, 9$ which satisfy on-shell constraint $k_{\mu} \epsilon^{\mu}=0$ :

$$
V^{(-1)}(\sigma) \equiv \epsilon_{\mu} \psi^{\mu}(\sigma) e^{-\phi(\sigma)} e^{i k \cdot X(\sigma)}, \quad V^{(0)}(\sigma) \equiv \epsilon_{\mu}\left(P^{\mu}(\sigma)+(k \cdot \psi) \psi^{\mu}(\sigma)\right) e^{i k \cdot X(\sigma)} .
$$

The superscripts indicate the superghost charges ( -1 and 0$)$, and refer to the contributions from the superghost system by means of a chiral boson $\phi[80,81]$. We work in conventions where the factors of $\bar{\delta}(k \cdot P(\sigma))$ enforcing scattering equations [28] are attributed to the integration measure in (1.1) when assembling amplitudes from the correlators in this section.

We also introduce the vertex operators for the gluino in $D=10$ spacetime dimensions

$$
V^{(-1 / 2)}(\sigma) \equiv 2^{-\frac{1}{4}} \chi^{\alpha} S_{\alpha}(\sigma) e^{-\frac{\phi(\sigma)}{2}} e^{i k \cdot X(\sigma)}, \quad V^{(-3 / 2)}(\sigma) \equiv 2^{\frac{1}{4}} \xi_{\alpha} S^{\alpha}(\sigma) e^{-\frac{3 \phi(\sigma)}{2}} e^{i k \cdot X(\sigma)},
$$

where the superghost charges are $-\frac{1}{2}$ and $-\frac{3}{2}$, respectively, and the normalization factors $2^{ \pm \frac{1}{4}}$ are chosen for later convenience. The fermion wave function $\chi^{\alpha}$ obeys the on-shell constraint $k_{\mu} \gamma_{\alpha \beta}^{\mu} \chi^{\beta}=0$, where Weyl-spinor indices $\alpha, \beta=1,2, \ldots, 16$ in an uppercase and lowercase position are left-handed and right-handed, respectively. The dual wave function $\xi_{\alpha}$ in the expression $(2.2)$ for $V^{(-3 / 2)}(\sigma)$ is defined to reproduce

$$
\chi^{\alpha}=k^{\mu} \gamma_{\mu}^{\alpha \beta} \xi_{\beta}
$$

Note that $P^{\mu}$ and $\psi^{\mu}$ are the free worldsheet fields of the RNS model, and $S_{\alpha}$ is the spin field $[82,83]$ (all depending on a puncture $\sigma$ on a Riemann sphere). Their operatorproduct expansions (OPEs) and the resulting techniques to evaluate tree-level correlators of the vertex operators (2.1) and (2.2) are collected in appendix B.1. 


\subsection{Tree-level correlator for external bosons}

Given gluon vertex operators, one can compute the tree-level correlator for $n$ bosons

$$
\mathcal{I}_{\text {bos }}^{\text {tree }}(1,2, \ldots, n)=\left\langle V_{1}^{(-1)}\left(\sigma_{1}\right) V_{2}^{(0)}\left(\sigma_{2}\right) V_{3}^{(0)}\left(\sigma_{3}\right) \ldots V_{n-1}^{(0)}\left(\sigma_{n-1}\right) V_{n}^{(-1)}\left(\sigma_{n}\right)\right\rangle,
$$

where we have chosen two legs, 1 and $n$, to have -1 superghost charges. Correlators of this type serve as half-integrands in the CHY formula (1.1) for tree amplitudes. A remarkable feature of the correlator (2.4) is that on the support of scattering equations, it is equivalent to the well-known (reduced) Pfaffian

$$
\mathcal{I}_{\text {bos }}^{\text {tree }}(1,2, \ldots, n)=\frac{1}{\sigma_{1, n}} \operatorname{Pf}\left|\Psi_{\{12 \ldots n\}}(\{\sigma, k, \epsilon\})\right|_{1, n}, \quad \sigma_{i, j} \equiv \sigma_{i}-\sigma_{j} .
$$

The $2 n \times 2 n$ antisymmetric matrix $\Psi$ was first introduced in [25], with columns and rows labelled by the $n$ momenta $k_{i}$ and polarizations $\epsilon_{i}$ for $i=1,2, \cdots, n$, and it also depends on the punctures $\sigma_{i}$. The entries of $\Psi$ are reviewed in appendix B.2 to fix our conventions.

The reduced Pfaffian $\mathrm{Pf}|\ldots|_{1, n}$ in (2.5) is defined by deleting two rows and columns $1, n$ of the matrix $\Psi$ with a prefactor $1 / \sigma_{1, n}$. More generally, one can define it by deleting any two columns and rows $1 \leq i<j \leq n$ and inserting a prefactor $(-1)^{i+j+n-1} / \sigma_{i, j}$ : this amounts to having the gluons $i, j$ the -1 picture, and while the correlator is manifestly symmetric in the remaining $n-2$ particles, on the support of scattering equations it becomes independent of $i, j$ thus completely symmetric as required by Bose symmetry.

By the definition of the Pfaffian, one can derive a useful (recursive) expansion, which was originally considered in [84] and used extensively in e.g. [66, 71]:

$$
\mathcal{I}_{\text {bos }}^{\text {tree }}(1,2, \ldots, n)=\sum_{\substack{\{23 \ldots n-1\} \\=A \cup B}} \operatorname{Pf}\left(\Psi_{A}\right) \sum_{\rho \in S_{|B|}} \operatorname{PT}(1, \rho(B), n)\left(\epsilon_{1} \cdot f_{\rho\left(b_{1}\right)} \cdot f_{\rho\left(b_{2}\right)} \cdot \ldots \cdot f_{\rho\left(b_{|B|}\right)} \cdot \epsilon_{n}\right) .
$$

Here the notation $\{2,3, \ldots, n-1\}=A \cup B$ in (2.6) instructs to sum over all the $2^{n-2}$ splittings of the set $\{2,3, \ldots, n-1\}$ into disjoint sets $A$ and $B$ with $|A|$ and $|B|$ elements. In each term, we have the Pfaffian of the matrix with particle labels in $A$ (which is of size $2|A| \times 2|A|$ ), times a sum over permutations $\rho \in S_{|B|}$ of labels in the complement $B \equiv\left\{b_{1}, b_{2}, \cdots, b_{|B|}\right\}$. We define $\operatorname{Pf} \Psi_{\emptyset}=1$ for the case of empty $A$. Moreover, (2.6) features Parke-Taylor factors

$$
\operatorname{PT}(1,2, \cdots, n)=\frac{1}{\sigma_{12} \sigma_{23} \ldots \sigma_{n-1, n} \sigma_{n, 1}}
$$

in the cyclic ordering $(1, \rho(B), n)=\left(1, \rho\left(b_{1}\right), \rho\left(b_{2}\right), \ldots, \rho\left(b_{|B|}\right), n\right)$. Finally, the kinematic coefficient of the Parke-Taylor factors in (2.6) are Lorentz contraction of $\epsilon_{1}, \epsilon_{n}$ and linearized field strengths

$$
f_{j}^{\mu \nu}=k_{j}^{\mu} \epsilon_{j}^{\nu}-k_{j}^{\nu} \epsilon_{j}^{\mu} .
$$

The dot products in $\left(\epsilon_{1} \cdot f_{\rho\left(b_{1}\right)} \cdot \ldots \cdot f_{\rho\left(b_{|B|}\right)} \cdot \epsilon_{n}\right)$ are understood in the sense of matrix multiplication, e.g. $\left(\epsilon_{1} \cdot f_{2} \cdot \epsilon_{n}\right)=\epsilon_{1}^{\mu}\left(f_{2}\right)_{\mu \nu} \epsilon_{n}^{\nu}$, so we reproduce the well-known three-point example

$$
\mathcal{I}_{\text {bos }}^{\text {tree }}(1,2,3)=\frac{\left(k_{3} \cdot \epsilon_{2}\right)\left(\epsilon_{1} \cdot \epsilon_{3}\right)+\left(\epsilon_{1} \cdot k_{2}\right)\left(\epsilon_{2} \cdot \epsilon_{3}\right)-\left(\epsilon_{1} \cdot \epsilon_{2}\right)\left(k_{2} \cdot \epsilon_{3}\right)}{\sigma_{1,2} \sigma_{2,3} \sigma_{3,1}} .
$$


At $n=4$, for instance, $\sum_{\{23\}=A \cup B}$ yields four contributions with $(A, B)=(\{2,3\}, \emptyset)$, $(\{2\},\{3\}),(\{3\},\{2\})$ and $(\emptyset,\{2,3\})$, which are given by

$$
\operatorname{Pf} \Psi_{\{2,3\}} \frac{\epsilon_{1} \cdot \epsilon_{4}}{\sigma_{1,4} \sigma_{4,1}}, \quad \operatorname{Pf} \Psi_{\{2\}} \frac{\epsilon_{1} \cdot f_{3} \cdot \epsilon_{4}}{\sigma_{1,3} \sigma_{3,4} \sigma_{4,1}}, \quad(2 \leftrightarrow 3), \quad \frac{\epsilon_{1} \cdot f_{2} \cdot f_{3} \cdot \epsilon_{4}}{\sigma_{1,2} \sigma_{2,3} \sigma_{3,4} \sigma_{4,1}}+(2 \leftrightarrow 3),
$$

respectively.

It has been known since [26] that using scattering equations, one can expand the correlator as a linear combination of Parke-Taylor factors, say in the partial-fraction independent set $\left\{\mathrm{PT}(1, \sigma(2,3, \cdots, n-1), n), \sigma \in S_{n-2}\right\}$, and the coefficients are BCJ master numerators for the corresponding $(n-2)$ ! half-ladder diagrams. One way of doing so is to start from (2.6), and the challenge is identical to extracting BCJ numerators for single-trace $\left(\mathrm{YM}+\phi^{3}\right)$ amplitudes. See [35, 68, 70-72] for more details.

\subsection{Tree-level correlator for two external fermions}

In the subsequent, we will cast two-fermion correlators involving two spin fields $S_{\alpha}[82,83]$ into simple forms by virtue of the current algebra generated by $\psi^{\mu} \psi^{\nu}$ along the lines of [85]. Note that such simplifications are partly motivated by (2.6) since such a correlator with external fermions can also be expanded in a similar form.

In the first representation, we have the two fermions, say, leg 1 and $n-1$, both in the $-\frac{1}{2}$ ghost picture, and one of the gluons, say leg $n$, in the -1 picture. Throughout this work, we will use the subscript "f" to denote fermions (gluinos) and suppress any subscript for the vector bosons (gluons). On the support of scattering equations, one can show that the tree-level correlator can be simplified to (see appendix B.3 for details)

$$
\begin{aligned}
\mathcal{I}_{2 \mathrm{f}}^{\text {tree }} & \left(1_{\mathrm{f}}, 2, \ldots,(n-2),(n-1)_{\mathrm{f}}, \hat{n}\right) \\
= & \left\langle V_{1}^{(-1 / 2)}\left(\sigma_{1}\right) V_{2}^{(0)}\left(\sigma_{2}\right) V_{3}^{(0)}\left(\sigma_{3}\right) \ldots V_{n-2}^{(0)}\left(\sigma_{n-2}\right) V_{n-1}^{(-1 / 2)}\left(\sigma_{n-1}\right) V_{n}^{(-1)}\left(\sigma_{n}\right)\right\rangle \\
= & \frac{1}{2} \sum_{\substack{\{23 \ldots n-2\} \\
=A \cup B \cup C}} \operatorname{Pf}\left(\Psi_{A}\right) \sum_{\rho \in S_{|B|}} \sum_{\tau \in S_{|C|}} \operatorname{PT}(1, \rho(B), n, \tau(C), n-1) \\
& \times\left(\chi_{1} f_{\rho\left(b_{1}\right)} f_{\rho\left(b_{2}\right)} \ldots f_{\rho\left(b_{|B|}\right)} \notin_{n} f_{\tau\left(c_{1}\right)} f_{\tau\left(c_{2}\right)} \ldots f_{\tau\left(c_{|C|}\right)} \chi_{n-1}\right),
\end{aligned}
$$

where we sum over all the splittings of the set $\{2,3, \cdots, n-2\}$ into disjoint sets $A, B$ and $C$, with again $\operatorname{Pf}\left(\Psi_{A}\right)$ times a sum over permutations $\rho$ and $\tau$ of the labels in $B$ and $C$, respectively. Similar to $(2.6)$, we have a Parke-Taylor factor $\mathrm{PT}(1, \rho(B), n, \tau(C), n-1)$ defined by (2.7) for each term. The main difference is that instead of the vector-index contraction, the linearized field strengths (2.8) are now contracted into gamma matrices. More specifically, with the conventions

$$
\notin_{n}=\epsilon_{n}^{\mu} \gamma_{\mu}, \quad f_{j}=\frac{1}{4} f_{j}^{\mu \nu} \gamma_{\mu \nu}=\frac{1}{2} k_{j}^{\mu} \epsilon_{j}^{\nu} \gamma_{\mu \nu}=\frac{1}{2} \not k_{j} \notin_{j},
$$

the last line of (2.11) features gamma-matrix products with the gluons in $\rho(B), \tau(C)$ entering via $f_{j}$, gluon $n$ entering via $\notin_{n}$, and the fermion wavefunctions $\chi_{1}, \chi_{n-1}$ contracting the free spinor induces, e.g. $\left(\chi_{1} f_{2} \notin_{n} \chi_{n-1}\right)=\frac{1}{4}\left(\chi_{1} \gamma_{\mu \nu} \gamma_{\lambda} \chi_{n-1}\right) f_{2}^{\mu \nu} \epsilon_{n}^{\lambda}$. In view of their contractions with Weyl spinors $\chi_{1}, \chi_{n-1}$, the gamma matrices in (2.11) are $16 \times 16$ Weyl-blocks 
within the Dirac matrices in 10 dimensions. Our conventions for their Clifford algebra and antisymmetric products are

$$
\gamma^{\mu} \gamma^{\nu}+\gamma^{\nu} \gamma^{\mu}=2 \eta^{\mu \nu}, \quad \gamma^{\mu \nu} \equiv \frac{1}{2}\left(\gamma^{\mu} \gamma^{\nu}-\gamma^{\nu} \gamma^{\mu}\right)
$$

A variant of (2.11) with $\notin_{n}$ moved adjacent to $\chi_{n-1}$ has been studied by Frost [86] along with its implication for the forward limit in the fermions.

At $n=3$ points, the two-fermion correlator (2.11) specializes to

$$
\mathcal{I}_{2 \mathrm{f}}^{\text {tree }}\left(1_{\mathrm{f}}, 2_{\mathrm{f}}, \hat{3}\right)=\frac{\left(\chi_{1} \phi_{3} \chi_{2}\right)}{2 \sigma_{1,3} \sigma_{3,2} \sigma_{2,1}},
$$

and the sum over $\{2\}=A \cup B \cup C$ in its ( $n=4)$-point instance gives rise to the following three terms instead of the four terms in the bosonic correlator (2.10) (also see [30]):

$$
\operatorname{Pf} \Psi_{\{2\}} \frac{\left(\chi_{1} \phi_{4} \chi_{3}\right)}{2 \sigma_{1,4} \sigma_{4,3} \sigma_{3,1}}, \quad \frac{\left(\chi_{1} f_{2} \phi_{4} \chi_{3}\right)}{2 \sigma_{1,2} \sigma_{2,4} \sigma_{4,3} \sigma_{3,1}}, \quad \frac{\left(\chi_{1} \phi_{4} f_{2} \chi_{3}\right)}{2 \sigma_{1,4} \sigma_{4,2} \sigma_{2,3} \sigma_{3,1}}
$$

The formula (2.11) for the two-fermion correlator is manifestly symmetric in most of the gluons $2,3, \cdots, n-2$ except for the last one $n$ which is earmarked through the hat notation in $\mathcal{I}_{2 \mathrm{f}}^{\text {tree }}(\ldots, \hat{n})$. On the support of scattering equations and the kinematic phase space of $n$ massless particles, one can show that (2.11) is also symmetric in all of $\hat{n}$ and $2,3, \ldots, n-2$. But this no longer the case in the forward-limit situation of section 5 , where we extract parity-odd contributions to one-loop correlators from (2.11).

Note that the expression (2.11) for the two-fermion correlator can be straightforwardly generalized to any even spacetime dimension since the structure of the underlying spinfield correlators is universal (see appendix B.3). However, only $D=2 \bmod 4$ admit $\chi_{1}$ and $\chi_{n-1}$ of the same chirality since the charge-conjugation matrix in these dimensions is off-diagonal in its $2^{D / 2-1} \times 2^{D / 2-1}$ Weyl blocks. In order to extend (2.11) to $D=0 \bmod 4$ dimensions, $\chi_{1}$ and $\chi_{n-1}$ need to be promoted to Weyl spinors of opposite chirality.

\subsection{Alternative representation of the two-fermion correlator}

In this section, we present an alternative representation of the two-fermion correlator which is manifestly symmetric in all its $n-2$ gluons. To do that, we put the two fermions, say leg 1 and $n$, in the $-\frac{1}{2}$ and $-\frac{3}{2}$ picture, respectively, and on the support of scattering equations we find (see appendix B.3 for details)

$\mathcal{I}_{2 \mathrm{f}}^{\text {tree }}\left(1_{\mathrm{f}}, 2, \ldots,(n-1), n_{\mathrm{f}}\right)=\left\langle V_{1}^{(-1 / 2)}\left(\sigma_{1}\right) V_{2}^{(0)}\left(\sigma_{2}\right) V_{3}^{(0)}\left(\sigma_{3}\right) \ldots V_{n-1}^{(0)}\left(\sigma_{n-1}\right) V_{n}^{(-3 / 2)}\left(\sigma_{n}\right)\right\rangle$

$$
=\sum_{\substack{\{23 \ldots n-1\} \\=A \cup B}} \operatorname{Pf}\left(\Psi_{A}\right) \sum_{\rho \in S_{|B|}} \operatorname{PT}(1, \rho(B), n)\left(\chi_{1} f_{\rho\left(b_{1}\right)} f_{\rho\left(b_{2}\right)} \ldots f_{\rho\left(b_{|B|}\right)} \xi_{n}\right)
$$

which takes a form even closer to (2.6) since we also sum over partitions $\{2,3, \cdots, n-1\}=$ $A \cup B$ with disjoint $A, B$. All the (gamma-matrix contracted) field strengths (2.12) in $\rho(B)$ are sandwiched between $\chi_{1}$ and $\xi_{n}$. 
At $n=3$, the sum over $\{2\}=A \cup B$ in (2.16) involves two terms:

$$
\begin{aligned}
\mathcal{I}_{2 \mathrm{f}}^{\text {tree }}\left(1_{\mathrm{f}}, 2,3_{\mathrm{f}}\right) & =\operatorname{Pf} \Psi_{\{2\}} \frac{\chi_{1} \xi_{3}}{\sigma_{1,3} \sigma_{3,1}}+\frac{\chi_{1} f_{2} \xi_{3}}{\sigma_{1,2} \sigma_{2,3} \sigma_{3,1}} \\
& =\frac{-1}{\sigma_{1,2} \sigma_{2,3} \sigma_{3,1}}\left\{\left(\epsilon_{2} \cdot k_{1}\right)\left(\chi_{1} \xi_{3}\right)+\frac{1}{2}\left(\chi_{1} \not k_{3} \phi_{2} \xi_{3}\right)\right\}
\end{aligned}
$$

In order to relate this to the earlier result (2.14) for the fermionic three-point correlator, we have rewritten $\operatorname{Pf} \Psi_{\{2\}}=\frac{\left(\epsilon_{2} \cdot k_{1}\right) \sigma_{1,3}}{\sigma_{2,1} \sigma_{2,3}}$ and $\chi_{1} f_{2} \xi_{3}=-\frac{1}{2} \chi_{1} k_{3} \notin_{2} \xi_{3}$ in passing to the second line. These identities are based on both momentum conservation and the physical-state conditions $\epsilon_{2} \cdot k_{2}=\chi_{1} k_{1}=0$. Finally, the Clifford algebra (2.13) gives rise to $\chi_{1} k_{3} /$ $\epsilon_{2} \xi_{3}=2\left(\epsilon_{2} \cdot k_{3}\right) \chi_{1} \xi_{3}-\chi_{1} \notin_{2} \not_{3} \xi_{3}$, and one can identify the wavefunction $\chi_{3}=\not k_{3} \xi_{3}$ by $(2.3)$. In this way, we reproduce the permutation

$$
\mathcal{I}_{2 \mathrm{f}}^{\text {tree }}\left(1_{\mathrm{f}}, 2,3_{\mathrm{f}}\right)=\frac{\left(\chi_{1} \phi_{2} \chi_{3}\right)}{2 \sigma_{1,2} \sigma_{2,3} \sigma_{3,1}}
$$

of the earlier three-point result (2.14). Even though this may appear to be a detour in the computation of the three-point correlator, the similarity of (2.16) with the bosonic correlator (2.6) will be a crucial benefit for the computation of forward limits.

At $n=4$ we have the four contributions similar to (2.10):

$$
\operatorname{Pf} \Psi_{\{2,3\}} \frac{\chi_{1} \xi_{4}}{\sigma_{1,4} \sigma_{4,1}}, \quad \operatorname{Pf} \Psi_{\{2\}} \frac{\chi_{1} f_{3} \xi_{4}}{\sigma_{1,3} \sigma_{3,4} \sigma_{4,1}}, \quad(2 \leftrightarrow 3), \quad \frac{\chi_{1} f_{2} f_{3} \xi_{4}}{\sigma_{1,2} \sigma_{2,3} \sigma_{3,4} \sigma_{4,1}}+(2 \leftrightarrow 3) .
$$

We remark that again we can further expand the $\operatorname{Pf} \Psi_{A}$ in both cases, and on the support of scattering equations eventually one can expand the correlator as a linear combination of (length- $n$ ) Parke-Taylor factors. Their coefficients can be identified with BCJ numerators [26, 68, 70, 71], now involving two external fermions on top of $n-2$ bosons. In the following, we will mostly work with the second representation (2.16) of the two-fermion correlator when we take the forward limit in the two fermions and combine it with the bosonic forward limit of (2.6). The parity-odd part of one-loop numerators in chiral theories in turn will be derived from the first representation (2.11) of the two-fermion correlator, see section 5 .

Similar to the results of the previous section, the two-fermion correlator (2.16) generalizes to any even spacetime dimension. The chiralities of $\chi_{1}$ and $\xi_{n}$ remain opposite in any $D=2 \bmod 4$, whereas dimensions $D=0 \bmod 4$ require a chirality flip in one of $\chi_{1}$ or $\xi_{n}$.

As detailed in appendix B.4, four-fermion correlators with any number of bosons can be brought into a very similar form. Six or more fermions, however, necessitate vertex operators in the $+1 / 2$ superghost picture that feature excited spin fields and give rise to more complicated $n$-point correlators [87-89]. Still, the results are available from the manifestly supersymmetric pure-spinor formalism [90], where $n$-point correlators in Parke-Taylor form are available in superspace $[13,14]$. Their components for arbitrary combinations of bosons and fermions can be conveniently extracted through the techniques of $[60,61]$. 


\subsection{Forward limits and gluing operators}

Finally, we review the prescription for taking forward limits in a pair of legs, which can be both bosons or both fermions. The momenta of the two legs are $+\ell$ and $-\ell$, respectively, which should be taken off shell, i.e. $\ell^{2} \neq 0 .{ }^{7}$ Moreover, we need to sum over the polarization states and other quantum numbers of the two legs. For example, since we consider all particles (both gluons and gluinos) to be in the adjoint representation of e.g. $U(N)$ color group, we have to sum over the $U(N)$ degrees of freedom of the pair of legs. In this way, the one-loop color-stripped amplitude can be obtained by summing over tree-level ones with the two adjacent legs inserted in all possible positions. This is the origin of the one-loop Parke-Taylor factors (A.4), also see $[38,45]$ for more details.

We shall now define the kinematic prescription for forward limits in two bosonic or fermionic legs. For that in bosonic legs $i$ and $j$, we define

$$
\epsilon_{i}^{\mu} \epsilon_{j}^{\nu} \rightarrow \mathrm{FWL}_{i, j}\left(\epsilon_{i}^{\mu} \epsilon_{j}^{\nu}\right)=\eta^{\mu \nu}-\ell^{\mu} \bar{\ell}^{\nu}-\ell^{\nu} \bar{\ell}^{\mu}, \quad \mathrm{FWL}_{i, j}\left(k_{i}, k_{j}\right)=(+\ell,-\ell)
$$

with an auxiliary vector $\bar{\ell}^{\mu}$ subject to $\ell \cdot \bar{\ell}=1$. Note that we have used the completeness relation of polarization vectors.

For the forward limit in fermionic legs $i$ and $j$, we define

$$
\left(\chi_{i}\right)^{\alpha}\left(\xi_{j}\right)_{\beta} \rightarrow \mathrm{FWL}_{i, j}\left(\left(\chi_{i}\right)^{\alpha}\left(\xi_{j}\right)_{\beta}\right)=\delta_{\beta}^{\alpha}, \quad \mathrm{FWL}_{i, j}\left(k_{i}, k_{j}\right)=(+\ell,-\ell),
$$

where we have used the completeness relation for fermion wave functions. When applied to a pair of vertex operators with total superghost charge -2 , the prescriptions (2.20) and (2.21) implement the gluing operators of Roehrig and Skinner [65].

Before proceeding, we remark that after taking the forward limit in a pair of gluons/gluinos in the tree-level correlator, (2.6) and (2.16), the only explicit dependence on loop momentum $\ell$ is in $\operatorname{Pf} \Psi_{A}$ through diagonal entries of the submatrix $C_{A}$; there is no loop momentum in other parts of $\operatorname{Pf} \Psi_{A}$ or factors involving particles in $B$. We will see in the subsequent that this observation immediately yields the power counting of loop momentum for BCJ numerators in various gauge theories.

\section{One loop correlators and numerators of ten-dimensional SYM}

In this section, we study one-loop correlators with external bosons for ten-dimensional SYM, which in turn give explicit BCJ numerators at one-loop level. We begin by taking the forward limit of tree-level correlators with two additional bosons and fermions, (2.6) and (2.16), respectively; in order to combine them, we present a key result of the section, namely a formula to express a spinor trace with any number of particles in terms of vector traces. Moreover, the relative coefficient is fixed by maximal supersymmetry, thus we can write a formula for the one-loop correlator with all the supersymmetry cancellations manifest at any multiplicity.

\footnotetext{
${ }^{7}$ This can be realized by allowing only these two momenta to have non-vanishing components in certain extra dimension. For example, in $D+1$ dimensions the momenta for the two additional legs are taken to be $\pm(\ell ;|\ell|)$, while those for others are $\left(k_{i} ; 0\right)$ for $i=1,2, \cdots, n$.
} 
Even though this section is dedicated to ten-dimensional SYM, we will retain a variable number $D$ of spacetime dimensions in various intermediate steps. This is done in preparation for the analogous discussion of lower-dimensional gauge theories in section 4 and justified by the universality of the form (2.16) of two-fermion correlators.

\subsection{The forward limit of two bosons/fermions}

Implementing the forward limits (2.20) and (2.21) via gluing operators [65] sends the presentation (2.6) and (2.16) of the tree-level correlators to

$$
\begin{aligned}
& \mathrm{FWL}_{1, n}\left[\mathcal{I}_{\text {bos }}^{\text {tree }}(1,2, \ldots, n)\right]=\sum_{\substack{\{23 \ldots n-1\} \\
=A \cup B}} \operatorname{Pf}\left(\Psi_{A}\right) \sum_{\rho \in S_{|B|}} \operatorname{PT}(1, \rho(B), n) \\
& \times\left\{\begin{array}{cc}
(D-2) & : B=\emptyset \\
\left(f_{\rho\left(b_{1}\right)} \cdot f_{\rho\left(b_{2}\right)} \cdot \ldots \cdot f_{\rho\left(b_{|B|}\right)}\right)^{\mu \nu} \eta_{\mu \nu} & : B \neq \emptyset
\end{array}\right. \\
& \mathrm{FWL}_{1, n}\left[\mathcal{I}_{2 \mathrm{f}}^{\text {tree }}\left(1_{\mathrm{f}}, 2, \ldots, n-1, n_{\mathrm{f}}\right)\right]=\sum_{\substack{\{23 \ldots n-1\} \\
=A \cup B}} \operatorname{Pf}\left(\Psi_{A}\right) \sum_{\rho \in S_{|B|}} \operatorname{PT}(1, \rho(B), n) \\
& \times\left\{\begin{array}{cc}
2^{D / 2-1} & : B=\emptyset \\
\left(f_{\rho\left(b_{1}\right)} f_{\rho\left(b_{2}\right)} \cdots f_{\rho\left(b_{|B|}\right)}\right) \alpha^{\beta} \delta_{\beta}^{\alpha}: & B \neq \emptyset
\end{array} .\right.
\end{aligned}
$$

The contribution of $B=\emptyset$ stems from contractions $\eta_{\mu \nu}\left(\eta^{\mu \nu}-\ell^{\mu} \bar{\ell}^{\nu}-\ell^{\nu} \bar{\ell}^{\mu}\right)=D-2$ and $\delta_{\beta}^{\alpha} \delta_{\alpha}^{\beta}=2^{D / 2-1}$ in (2.20) and (2.21), the latter being the dimension of a chiral spinor representation in even spacetime dimensions $D$. In spelling out the contributions of $B \neq \emptyset$ to the bosonic forward limit, we have exploited that the terms $\sim \ell^{\mu} \bar{\ell}^{\nu}+\ell^{\nu} \bar{\ell}^{\mu}$ in $(2.20)$ do not contribute upon contraction of with vectors different from $\epsilon_{i}, \epsilon_{j}[65]$.

We shall introduce some notation for the frequently reoccurring traces over vector and spinor indices, relegating the discussion of parity-odd pieces to section 5 :

$$
\begin{aligned}
\operatorname{tr}_{\mathrm{V}}(1,2, \ldots, p) & =\left(f_{1} \cdot f_{2} \cdot \ldots \cdot f_{p}\right)^{\mu \nu} \eta_{\mu \nu}=\left(f_{1}\right)^{\mu_{1}} \mu_{2}\left(f_{2}\right)^{\mu_{2}}{ }_{\mu_{3}} \ldots\left(f_{p}\right)^{\mu_{p}}{ }_{\mu_{1}} \\
\operatorname{tr}_{\mathrm{S}}(1,2, \ldots, p) & =\left.\left(f_{1} f_{2} \ldots f_{p}\right)_{\alpha}^{\beta} \delta_{\beta}^{\alpha}\right|_{\text {even }} \\
& =\left.\frac{1}{4^{p}} f_{1}^{\mu_{1} \nu_{1}} f_{2}^{\mu_{2} \nu_{2}} \ldots f_{p}^{\mu_{p} \nu_{p}}\left(\gamma_{\mu_{1} \nu_{1}}\right)_{\alpha_{1}}{ }^{\alpha_{2}}\left(\gamma_{\mu_{2} \nu_{2}}\right)_{\alpha_{2}}{ }^{\alpha_{3}} \ldots\left(\gamma_{\mu_{p} \nu_{p}}\right)_{\alpha_{p}}{ }^{\alpha_{1}}\right|_{\text {even }}
\end{aligned}
$$

We remark that the spinor trace in (3.2) would in principle contain parity-odd terms, but here we define $\operatorname{tr}_{S}(1,2, \ldots, p)$ to be the parity-even part by manually discarding parity-odd terms. ${ }^{8}$ Note that the $B=\emptyset$ contribution to (3.2) formally arises from $\operatorname{tr}_{\mathrm{S}}(\emptyset)=2^{D / 2-1}$, and non-empty traces are cyclic and exhibit the parity properties

$$
\operatorname{tr}_{\mathrm{V}}(1,2, \ldots, p)=(-1)^{p} \operatorname{tr}_{\mathrm{V}}(p, \ldots, 2,1), \quad \operatorname{tr}_{\mathrm{S}}(1,2, \ldots, p)=(-1)^{p} \operatorname{tr}_{\mathrm{S}}(p, \ldots, 2,1) \text {. }
$$

In order to study the supersymmetry cancellations in one-loop correlators, we will be interested in linear combinations of bosonic and fermionic forward limits with theorydependent relative weights. The main results of this work are driven by the observation

\footnotetext{
${ }^{8}$ For $n=5$, the parity-odd term $\varepsilon_{10}\left(f_{1}, f_{2}, f_{3}, f_{4}, f_{5}\right)$ in a chiral spinor trace vanishes by momentum conservation, in contrast to the one in (5.10) due to a different prescription.
} 
that most of the structure in (3.1) and (3.2) is preserved in combining bosons and fermions such that the linear combinations are taken at the level of the field-strength traces: with an a priori undetermined weight factor $\alpha \in \mathbb{Q}$, we have

$$
\begin{aligned}
& \mathcal{I}_{\text {bos }, \alpha}^{(1)}(1,2, \ldots, n)=\left.\mathrm{FWL}_{+,-}\left[\mathcal{I}_{\text {bos }}^{\text {tree }}(+, 1,2, \ldots, n,-)+\alpha \cdot \mathcal{I}_{2 \mathrm{f}}^{\text {tree }}\left(+_{f}, 1,2, \ldots, n,-{ }_{f}\right)\right]\right|_{\text {even }} \\
& =\sum_{\substack{\{12 \ldots n\} \\
=A \cup B}} \operatorname{Pf}\left(\Psi_{A}\right) \sum_{\rho \in S_{|B|}} \operatorname{PT}(+, \rho(B),-) \\
& \quad \times\left\{\begin{array}{cc}
: & B=\emptyset \\
{\left[\operatorname{tr}_{\mathrm{V}}\left(\rho\left(b_{1}\right), \rho\left(b_{2}\right), \ldots, \rho\left(b_{|B|}\right)\right)+\alpha \cdot \operatorname{tr}_{\mathrm{S}}\left(\rho\left(b_{1}\right), \rho\left(b_{2}\right), \ldots, \rho\left(b_{|B|}\right)\right)\right]:} & B \neq \emptyset
\end{array} .\right.
\end{aligned}
$$

The $B=\emptyset$ contribution $\sim \operatorname{Pf}\left(\Psi_{\{12 \ldots n\}}\right)$ will be proportional to at least one power of loop momentum since a plain Pfaffian in a tree-level context is known to vanish on the support of the scattering equations. The diagonal entries $C_{j j}$ in the expansion of $\operatorname{Pf}\left(\Psi_{A}\right)$ within (3.6) still involve terms $\epsilon_{j}^{\mu}\left(\frac{\ell_{\mu}}{\sigma_{j,+}}-\frac{\ell_{\mu}}{\sigma_{j,-}}\right)$ which would be absent in the naive tree-level incarnation of $\operatorname{Pf}\left(\Psi_{\{12 \ldots n\}}\right)$ without any reference to extra legs,+- .

\subsection{From spinor traces to vector ones}

In this subsection we propose the identities which allow us to convert any spinor trace to vector ones. Our result will be useful in the subsequent sections when we study the one-loop correlator and BCJ numerators for various gauge theories.

Our starting point is the well-known formula for traces of chiral gamma matrices

$$
\operatorname{tr}_{\mathrm{S}}\left(\gamma^{\mu \nu}\right)=0, \quad \operatorname{tr}_{\mathrm{S}}\left(\gamma^{\mu \nu} \gamma^{\lambda \rho}\right)=2^{D / 2-1}\left(\eta^{\nu \lambda} \eta^{\mu \rho}-\eta^{\mu \lambda} \eta^{\nu \rho}\right), \quad \text { etc. }
$$

We will review a recursion for such traces in appendix C.1, and based on that it is easy to show that $\operatorname{tr}_{\mathrm{S}}(\emptyset)=2^{D / 2-1}$ generalizes to

$$
\operatorname{tr}_{\mathrm{S}}(1)=0, \quad \operatorname{tr}_{\mathrm{S}}(1,2)=2^{D / 2-4} \operatorname{tr}_{\mathrm{V}}(1,2), \quad \operatorname{tr}_{\mathrm{S}}(1,2,3)=2^{D / 2-4} \operatorname{tr}_{\mathrm{V}}(1,2,3)
$$

where the numbers enclosed in (...) label the external particles according to our conventions (3.3) and (3.4). Starting from four points, more permutations appear: for $n=4$ the result is given by a sum of single traces and double traces w.r.t. vector indices,

$$
\begin{aligned}
\operatorname{tr}_{\mathrm{S}}(1,2,3,4)= & 2^{D / 2-5}\left\{\operatorname{tr}_{\mathrm{V}}(1,2,3,4)-\operatorname{tr}_{\mathrm{V}}(1,3,2,4)-\operatorname{tr}_{\mathrm{V}}(1,2,4,3)\right\} \\
& +2^{D / 2-7}\left\{\operatorname{tr}_{\mathrm{V}}(1,2) \operatorname{tr}_{\mathrm{V}}(3,4)+\operatorname{tr}_{\mathrm{V}}(1,3) \operatorname{tr}_{\mathrm{V}}(2,4)+\operatorname{tr}_{\mathrm{V}}(1,4) \operatorname{tr}_{\mathrm{V}}(2,3)\right\},
\end{aligned}
$$

where we have used the parity properties (3.5): for single-trace terms we have 6 (cyclically inequivalent) permutations but only 3 of them are independent under parity. 
Moving to the $n=5$ case, we find that $\operatorname{tr}_{\mathrm{S}}(1,2,3,4,5)$ is again given by combinations of single and double traces,

$$
\begin{aligned}
2^{D / 2-6} & \left\{\operatorname{tr}_{\mathrm{V}}(1,2,3,4,5)-\operatorname{tr}_{\mathrm{V}}(1,2,3,5,4)-\operatorname{tr}_{\mathrm{V}}(1,2,4,3,5)-\operatorname{tr}_{\mathrm{V}}(1,2,4,5,3)\right. \\
& -\operatorname{tr}_{\mathrm{V}}(1,2,5,3,4)+\operatorname{tr}_{\mathrm{V}}(1,2,5,4,3)-\operatorname{tr}_{\mathrm{V}}(1,3,2,4,5)+\operatorname{tr}_{\mathrm{V}}(1,3,2,5,4) \\
& \left.-\operatorname{tr}_{\mathrm{V}}(1,4,2,3,5)-\operatorname{tr}_{\mathrm{V}}(1,3,5,2,4)+\operatorname{tr}_{\mathrm{V}}(1,4,3,2,5)-\operatorname{tr}_{\mathrm{V}}(1,3,4,2,5)\right\} \\
+2^{D / 2-}- & \left\{\operatorname{tr}_{\mathrm{V}}(1,2) \operatorname{tr}_{\mathrm{V}}(3,4,5)+\operatorname{tr}_{\mathrm{V}}(1,3) \operatorname{tr}_{\mathrm{V}}(2,4,5)+\operatorname{tr}_{\mathrm{V}}(1,4) \operatorname{tr}_{\mathrm{V}}(2,3,5)+\operatorname{tr}_{\mathrm{V}}(1,5) \operatorname{tr}_{\mathrm{V}}(2,3,4)\right. \\
& +\operatorname{tr}_{\mathrm{V}}(2,3) \operatorname{tr}_{\mathrm{V}}(1,4,5)+\operatorname{tr}_{\mathrm{V}}(2,4) \operatorname{tr}_{\mathrm{V}}(1,3,5)+\operatorname{tr}_{\mathrm{V}}(2,5) \operatorname{tr}_{\mathrm{V}}(1,3,4)+\operatorname{tr}_{\mathrm{V}}(3,4) \operatorname{tr}_{\mathrm{V}}(1,2,5) \\
& \left.+\operatorname{tr}_{\mathrm{V}}(3,5) \operatorname{tr}_{\mathrm{V}}(1,2,4)+\operatorname{tr}_{\mathrm{V}}(4,5) \operatorname{tr}_{\mathrm{V}}(1,2,3)\right\},
\end{aligned}
$$

where only $4 ! / 2=12$ single-trace terms, and $\left(\begin{array}{l}5 \\ 2\end{array}\right)=10$ double-trace terms are independent under parity.

As we will show recursively in appendix C.1, in general the $n$-point spinor trace can be written as a sum of terms with $j=1,2, \cdots,\lfloor n / 2\rfloor$ vector traces with suitable prefactors,

$$
\operatorname{tr}_{\mathrm{S}}(1,2, \ldots, n)=2^{D / 2-1-n} \sum_{j=1}^{\lfloor n / 2\rfloor} \frac{1}{2^{j} j !} \sum_{\substack{\{12 \ldots n\} \\=A_{1} \cup A_{2} \cup \ldots \cup A_{j}}} \prod_{i=1}^{j}\left(\sum_{\sigma \in S_{\left|A_{i}\right|} / \mathbb{Z}_{\left|A_{i}\right|}} \operatorname{tr}_{\mathrm{V}}\left(\sigma\left(A_{i}\right)\right) \operatorname{ord}_{\sigma}^{\text {id }}\right)
$$

where for each $j$, we sum over partitions of $\{1,2, \ldots, n\}$ into $j$ disjoint subsets $A_{1}, A_{2}, \ldots, A_{j}$, and the factor $\frac{1}{j !}$ compensates for the overcounting of partitions due to permutations of $A_{1}, A_{2}, \ldots, A_{j}$; for each subset $A_{i}$ we sum over all cyclically inequivalent permutations $\sigma \in S_{\left|A_{i}\right|} / \mathbb{Z}_{\left|A_{i}\right|}$, e.g.by fixing the first element in $\operatorname{tr}_{\mathrm{V}}\left(\sigma\left(A_{i}\right)\right)$ to be the smallest one in $A_{i}$; finally the sign ord ${ }_{\sigma}^{i d}$ counts the number of descents in $\sigma$ (compared to the identity permutation). For example, $\operatorname{ord}_{132}^{i d}=-1$, ord $d_{1243}^{i d}=\operatorname{ord}_{1324}^{i d}=-1$, and $\operatorname{ord}_{1432}^{i d}=1$. An alternative representation of the parity-even spinor trace (3.4) in terms of a Pfaffian can be found in (4.35a) of [65].

More generally, if the spinor trace has an ordering $\rho$, one can choose the first element $\sigma_{1}$ to be the smallest in $\rho$, and the sign $\operatorname{ord}_{\sigma}^{\rho}$ can be factorized as

$$
\operatorname{ord}_{\sigma}^{\rho}=\operatorname{sgn}_{\sigma_{p}, \sigma_{p-1}}^{\rho} \operatorname{sgn}_{\sigma_{p-1}, \sigma_{p-2}}^{\rho} \ldots \operatorname{sgn}_{\sigma_{4}, \sigma_{3}}^{\rho} \operatorname{sgn}_{\sigma_{3}, \sigma_{2}}^{\rho}
$$

where the $\operatorname{sgn}_{i j}^{\rho}$ factors are defined to be \pm 1 according to the conventions of [44]

$$
\operatorname{sgn}_{i j}^{\rho}=\left\{\begin{array}{ll}
+1: & i \text { is on the right of } j \text { in } \rho(1,2, \ldots, p) \\
-1: & i \text { is on the left of } j \text { in } \rho(1,2, \ldots, p)
\end{array} .\right.
$$

For example, ord $1_{132}^{132}=1$ instead of ord $\mathrm{d}_{132}^{i d}=-1$ and $\operatorname{ord}_{1432}^{1243}=-1$. Let's end the discussion with an example for triple-trace contribution $(j=3)$ of $\operatorname{tr}_{S}(1,2,3,4,5,6)$, which reads

$$
\begin{aligned}
2^{D / 2-10} & \left\{\operatorname{tr}_{\mathrm{V}}(1,2)\left[\operatorname{tr}_{\mathrm{V}}(3,4) \operatorname{tr}_{\mathrm{V}}(5,6)+\operatorname{tr}_{\mathrm{V}}(3,5) \operatorname{tr}_{\mathrm{V}}(4,6)+\operatorname{tr}_{\mathrm{V}}(3,6) \operatorname{tr}_{\mathrm{V}}(4,5)\right]\right. \\
& +\operatorname{tr}_{\mathrm{V}}(1,3)\left[\operatorname{tr}_{\mathrm{V}}(2,5) \operatorname{tr}_{\mathrm{V}}(4,6)+\operatorname{tr}_{\mathrm{V}}(2,6) \operatorname{tr}_{\mathrm{V}}(4,5)+\operatorname{tr}_{\mathrm{V}}(2,5) \operatorname{tr}_{\mathrm{V}}(4,6)\right] \\
& +\operatorname{tr}_{\mathrm{V}}(1,4)\left[\operatorname{tr}_{\mathrm{V}}(2,3) \operatorname{tr}_{\mathrm{V}}(5,6)+\operatorname{tr}_{\mathrm{V}}(2,5) \operatorname{tr}_{\mathrm{V}}(3,6)+\operatorname{tr}_{\mathrm{V}}(2,6) \operatorname{tr}_{\mathrm{V}}(3,5)\right] \\
& +\operatorname{tr}_{\mathrm{V}}(1,5)\left[\operatorname{tr}_{\mathrm{V}}(2,3) \operatorname{tr}_{\mathrm{V}}(4,6)+\operatorname{tr}_{\mathrm{V}}(2,4) \operatorname{tr}_{\mathrm{V}}(3,6)+\operatorname{tr}_{\mathrm{V}}(2,6) \operatorname{tr}_{\mathrm{V}}(3,4)\right] \\
& \left.+\operatorname{tr}_{\mathrm{V}}(1,6)\left[\operatorname{tr}_{\mathrm{V}}(2,3) \operatorname{tr}_{\mathrm{V}}(4,5)+\operatorname{tr}_{\mathrm{V}}(2,4) \operatorname{tr}_{\mathrm{V}}(3,5)+\operatorname{tr}_{\mathrm{V}}(2,5) \operatorname{tr}_{\mathrm{V}}(3,4)\right]\right\} .
\end{aligned}
$$




\subsection{Ten-dimensional SYM}

Since we have not been careful about the normalization of the fermionic tree-level correlator (2.11), the normalization constant $\alpha$ in (3.6) for a single Weyl fermion will be fixed by the example of ten-dimensional SYM. The supersymmetry cancellations are wellknown to yield vanishing $(n \leq 3)$-point one-loop integrands in $D=10 \mathrm{SYM}$ [91]. Accordingly, there exists a choice $\alpha=-\frac{1}{2}$ in (3.6) such that both the $B=\emptyset$ contributions $D-2+\left.\alpha \cdot 2^{D / 2-1}\right|_{D=10}$ and those with $|B|=2,3$ vanish:

$$
\begin{aligned}
& \mathcal{I}_{D=10 \mathrm{SYM}}^{(1)}(1,2, \ldots, n)=\sum_{\substack{\{12 \ldots n\} \\
=A \cup B}} \operatorname{Pf}\left(\Psi_{A}\right) \sum_{\rho \in S_{|B|}} \operatorname{PT}(+, \rho(B),-) \\
& \quad \times\left\{\begin{array}{cc}
0 & :|B| \leq 3 \\
{\left[\operatorname{tr}_{\mathrm{V}}\left(\rho\left(b_{1}\right), \rho\left(b_{2}\right), \ldots, \rho\left(b_{|B|}\right)\right)-\frac{1}{2} \operatorname{tr}_{\mathrm{S}}\left(\rho\left(b_{1}\right), \rho\left(b_{2}\right), \ldots, \rho\left(b_{|B|}\right)\right)\right]} & :|B| \geq 4
\end{array} .\right.
\end{aligned}
$$

Recall that the spinor trace $\operatorname{tr}_{\mathrm{S}}$ is defined to contain the parity-even part only. We have used the relation (3.8) between vector and spinor two- and three-traces in $D=10$ dimensions, $\operatorname{tr}_{\mathrm{S}}(1,2)=2 \operatorname{tr}_{\mathrm{V}}(1,2)$ and $\operatorname{tr}_{\mathrm{S}}(1,2,3)=2 \operatorname{tr}_{\mathrm{V}}(1,2,3)$. Throughout this work, the external states of the one-loop correlators are gauge bosons. Thus we will no longer specify bos in the subscripts of $\mathcal{I}^{(1)}$.

The first non-vanishing contribution to (3.15) from the field strengths at $|B|=4$ turns out to not depend on the permutation $\rho$ and reproduces the famous $t_{8}$-tensor, cf. (3.9),

$$
\begin{aligned}
& \operatorname{tr}_{\mathrm{V}}(1,2,3,4)-\left.\frac{1}{2} \operatorname{tr}_{\mathrm{S}}(1,2,3,4)\right|_{D=10} \\
&=\operatorname{tr}_{\mathrm{V}}(1,2,3,4)-\frac{1}{2}\left\{\operatorname{tr}_{\mathrm{V}}(1,2,3,4)-\operatorname{tr}_{\mathrm{V}}(1,3,2,4)-\operatorname{tr}_{\mathrm{V}}(1,2,4,3)\right\} \\
&-\frac{1}{8}\left\{\operatorname{tr}_{\mathrm{V}}(1,2) \operatorname{tr}_{\mathrm{V}}(3,4)+\operatorname{tr}_{\mathrm{V}}(1,3) \operatorname{tr}_{\mathrm{V}}(2,4)+\operatorname{tr}_{\mathrm{V}}(1,4) \operatorname{tr}_{\mathrm{V}}(2,3)\right\} \\
&= \frac{1}{2} \operatorname{tr}_{\mathrm{V}}(1,2,3,4)-\frac{1}{8} \operatorname{tr}_{\mathrm{V}}(1,2) \operatorname{tr}_{\mathrm{V}}(3,4)+\operatorname{cyc}(2,3,4)=\frac{1}{2} t_{8}\left(f_{1}, f_{2}, f_{3}, f_{4}\right),
\end{aligned}
$$

which is known from one-loop four-point amplitudes of the superstring [91] and defined by

$$
\begin{aligned}
t_{8}\left(f_{1}, f_{2}, f_{3}, f_{4}\right)= & \operatorname{tr}_{\mathrm{V}}(1,2,3,4)+\operatorname{tr}_{\mathrm{V}}(1,3,2,4)+\operatorname{tr}_{\mathrm{V}}(1,2,4,3) \\
& -\frac{1}{4}\left\{\operatorname{tr}_{\mathrm{V}}(1,2) \operatorname{tr}_{\mathrm{V}}(3,4)+\operatorname{tr}_{\mathrm{V}}(1,3) \operatorname{tr}_{\mathrm{V}}(2,4)+\operatorname{tr}_{\mathrm{V}}(1,4) \operatorname{tr}_{\mathrm{V}}(2,3)\right\}
\end{aligned}
$$

Hence, the four-point instance of (3.15) is the well-known permutation symmetric combination of Parke-Taylor factors,

$$
\mathcal{I}_{D=10 \mathrm{SYM}}^{(1)}(1,2,3,4)=\frac{1}{2} t_{8}\left(f_{1}, f_{2}, f_{3}, f_{4}\right) \sum_{\rho \in S_{4}} \mathrm{PT}(+, \rho(1,2,3,4),-) .
$$

Starting at five points, we need the case with $|B|=5$, and a similar expression can be 
given

$$
\begin{aligned}
& \operatorname{tr}_{\mathrm{V}}(1,2,3,4,5)-\left.\frac{1}{2} \operatorname{tr}_{\mathrm{S}}(1,2,3,4,5)\right|_{D=10} \\
& =\frac{1}{4}\left\{3 \operatorname{tr}_{\mathrm{V}}(1,2,3,4,5)+\operatorname{tr}_{\mathrm{V}}(1,2,3,5,4)+\operatorname{tr}_{\mathrm{V}}(1,2,4,3,5)+\operatorname{tr}_{\mathrm{V}}(1,2,4,5,3)\right. \\
& \quad+\operatorname{tr}_{\mathrm{V}}(1,2,5,3,4)-\operatorname{tr}_{\mathrm{V}}(1,2,5,4,3)+\operatorname{tr}_{\mathrm{V}}(1,3,2,4,5)-\operatorname{tr}_{\mathrm{V}}(1,3,2,5,4) \\
& \left.\quad+\operatorname{tr}_{\mathrm{V}}(1,4,2,3,5)+\operatorname{tr}_{\mathrm{V}}(1,3,5,2,4)-\operatorname{tr}_{\mathrm{V}}(1,4,3,2,4)+\operatorname{tr}_{\mathrm{V}}(1,3,4,2,5)\right\} \\
& -\frac{1}{8}\left\{\operatorname{tr}_{\mathrm{V}}(1,2) \operatorname{tr}_{\mathrm{V}}(3,4,5)+\operatorname{tr}_{\mathrm{V}}(1,3) \operatorname{tr}_{\mathrm{V}}(2,4,5)+\operatorname{tr}_{\mathrm{V}}(1,4) \operatorname{tr}_{\mathrm{V}}(2,3,5)+\operatorname{tr}_{\mathrm{V}}(1,5) \operatorname{tr}_{\mathrm{V}}(2,3,4)\right. \\
& \quad+\operatorname{tr}_{\mathrm{V}}(2,3) \operatorname{tr}_{\mathrm{V}}(1,4,5)+\operatorname{tr}_{\mathrm{V}}(2,4) \operatorname{tr}_{\mathrm{V}}(1,3,5)+\operatorname{tr}_{\mathrm{V}}(2,5) \operatorname{tr}_{\mathrm{V}}(1,3,4)+\operatorname{tr}_{\mathrm{V}}(3,4) \operatorname{tr}_{\mathrm{V}}(1,2,5) \\
& \left.\quad+\operatorname{tr}_{\mathrm{V}}(3,5) \operatorname{tr}_{\mathrm{V}}(1,2,4)+\operatorname{tr}_{\mathrm{V}}(4,5) \operatorname{tr}_{\mathrm{V}}(1,2,3)\right\} \\
& =\frac{1}{4}\left\{t_{8}\left(\left[f_{1}, f_{2}\right], f_{3}, f_{4}, f_{5}\right)+t_{8}\left(\left[f_{1}, f_{3}\right], f_{2}, f_{4}, f_{5}\right)+t_{8}\left(\left[f_{1}, f_{4}\right], f_{2}, f_{3}, f_{5}\right)+t_{8}\left(\left[f_{1}, f_{5}\right], f_{2}, f_{3}, f_{4}\right)\right. \\
& \quad+t_{8}\left(\left[f_{2}, f_{3}\right], f_{1}, f_{4}, f_{5}\right)+t_{8}\left(\left[f_{2}, f_{4}\right], f_{1}, f_{3}, f_{5}\right)+t_{8}\left(\left[f_{2}, f_{5}\right], f_{1}, f_{3}, f_{4}\right)+t_{8}\left(\left[f_{3}, f_{4}\right], f_{1}, f_{2}, f_{5}\right) \\
& \left.\quad+t_{8}\left(\left[f_{3}, f_{5}\right], f_{1}, f_{2}, f_{4}\right)+t_{8}\left(\left[f_{4}, f_{5}\right], f_{1}, f_{2}, f_{3}\right)\right\},
\end{aligned}
$$

where we have used e.g. $\left[f_{1}, f_{2}\right]^{\mu \nu} \equiv f_{1}^{\mu} f_{2}^{\lambda \nu}-f_{2 \lambda}^{\mu} f_{1}^{\lambda \nu}$ inside the $t_{8}$ tensor. Let us already emphasize here that (3.15) after rewriting $\operatorname{tr}_{\mathrm{S}}(\ldots)$ in terms of $\operatorname{tr}_{\mathrm{V}}(\ldots)$ applies to any dimensional reduction of ten-dimensional SYM, for instance $\mathcal{N}=4 \mathrm{SYM}$ in $D=4$ (cf. section 4).

By analogy with (3.16), one may define higher-rank tensors beyond $t_{8}$ in (3.17). We can use the difference of vector and spinor traces to define higher-point extensions of (3.17) that will capture the kinematic factors besides the $\operatorname{Pf}\left(\Psi_{A}\right)$ in the correlators $(3.15) D=10$ SYM. As exemplified by the five-point case (3.19), higher-point $\operatorname{tr}_{\mathrm{V}}(\ldots)-\frac{1}{2} \operatorname{tr}_{\mathrm{S}}(\ldots)$ will involve $t_{8}$ tensors with nested commutators of $f_{j}$ w.r.t. Lorentz indices in its entries. The only new tensor structures that are not expressible in terms of $t_{8}$ with commutators arise from the permutation symmetric combination ${ }^{9}$

$$
\begin{aligned}
t_{2 n} & \left(f_{1}, f_{2}, \ldots, f_{n}\right) \\
\quad & =\left.\frac{1}{(n-1) !} \sum_{\rho \in S_{n-1}}\left[2 \operatorname{tr}_{\mathrm{V}}(1, \rho(2), \rho(3), \ldots, \rho(n))-\operatorname{tr}_{\mathrm{S}}(1, \rho(2), \rho(3), \ldots, \rho(n))\right]\right|_{D=10}
\end{aligned}
$$

involving an even number $n$ of field strengths. The permutation sum vanishes for odd $n$ by the parity properties (3.5). Rewriting correlators of $D=10 \mathrm{SYM}$ in terms of (3.20) is the kinematic analogue of decomposing color traces in gauge-theory amplitudes into contracted structure constants and symmetrized traces, where only the latter can furnish independent color tensors [92].

The simplest instance of (3.20) beyond $t_{8}$ is a rank-twelve tensor $t_{12}$ occurring at $n=6$. As detailed in appendix C.2, the case of $t_{12}$ admits an exceptional simplification that is not possible for $t_{16}$ and any higher-rank tensor (3.20): one can reduce $t_{12}$ to products,

$$
t_{12}\left(f_{1}, f_{2}, \ldots, f_{6}\right)=\frac{1}{24}\left[\operatorname{tr}_{\mathrm{V}}(1,2) t_{8}\left(f_{3}, f_{4}, f_{5}, f_{6}\right)+(1,2 \mid 1,2,3,4,5,6)\right]
$$

\footnotetext{
${ }^{9}$ At six points, for instance, $\operatorname{tr}_{\mathrm{V}}(1,2, \ldots, 6)-\frac{1}{2} \operatorname{tr}_{\mathrm{S}}(1,2, \ldots, 6)$ can be rewritten as its permutation symmetric part plus permutations of the two topologies $t_{8}\left(\left[f_{1}, f_{2}\right],\left[f_{3}, f_{4}\right], f_{5}, f_{6}\right)$ and $t_{8}\left(\left[\left[f_{1}, f_{2}\right], f_{3}\right], f_{4}, f_{5}, f_{6}\right)$.
} 
where the four-traces and products $\operatorname{tr}_{\mathrm{V}}\left(i_{1}, i_{2}\right) \operatorname{tr}_{\mathrm{V}}\left(i_{3}, i_{4}\right) \operatorname{tr}_{\mathrm{V}}\left(i_{5}, i_{6}\right)$ conspire to $t_{8}$. Here and throughout the rest of this work, the notation $+(1,2 \mid 1,2, \ldots, k)$ instructs to add all permutations of the preceding expression where the ordered pair of labels 1,2 is exchanged by any other pair $i, j \in\{1,2, \ldots, k\}$ with $i<j$. A similar notation $+(1,2, \ldots, j \mid 1,2, \ldots, k)$ with $j<k$ will be used to sum over all possibilities to pick $j$ elements from a sequence of $k$, for a total of $\left(\begin{array}{l}k \\ j\end{array}\right)$ terms.

The exceptional simplification of $t_{12}$ in (3.21) can be anticipated from the fact that sixtraces $\operatorname{tr}_{\mathrm{V}}(1,2, \ldots, 6)$ cancel from the combination (3.20) after rewriting the spinor traces via (3.11). For any higher-rank $t_{2 n}$ at $n \geq 8$ in turn, the coefficient of $\operatorname{tr}_{\mathrm{V}}(1,2, \ldots, n)$ is non-zero when expressing the spinor traces of (3.20) in terms of $\operatorname{tr}_{\mathrm{V}}(\ldots)$. These coefficients are worked out in terms of Eulerian numbers in appendix C.2.

In summary, the tensor structure of the $n$-point correlators (3.15) in $D=10 \mathrm{SYM}$ is captured by $\operatorname{Pf}\left(\Psi_{A}\right)$ and even-rank tensors $t_{2 n}$ in (3.20) including $t_{8}$ in (3.17) contracting nested commutators of field strengths.

\subsection{BCJ numerators versus single-trace $\mathrm{YM}+\phi^{3}$ at tree level}

Given the general formula (3.15) for the one-loop correlator in ten-dimensional SYM, one can read off the BCJ master numerators $N^{(1)}$ of an $n$-gon diagram as soon as all the $\sigma_{j}$-dependences of the Parke-Taylor factors and the $\operatorname{Pf}\left(\Psi_{A}\right)$ are lined up with

$$
\mathcal{I}^{(1)}(1,2, \ldots, n)=\frac{1}{2} \sum_{\omega \in S_{n}} \mathrm{PT}(+, \omega(1,2, \ldots, n),-) N^{(1)}(+, \omega(1,2, \ldots, n),-),
$$

where we need to use scattering equations at $(n+2)$ points. ${ }^{10}$ More specifically, the numerator $N^{(1)}(+, \omega(1,2, \ldots, n),-)$ refers to one of the $(n+2)$-point half-ladder diagrams in the right panel of figure 1 that arises from the partial-fraction decomposition of the $n$-gon propagators reviewed in appendix $\mathrm{A}$.

For a given partition $\{1,2, \ldots, n\}=A \cup B$ in (3.15), the leftover task is to absorb the $\sigma_{j}$-dependence of the Pfaffian into the $(|B|+2)$-point Parke-Taylor factors,

$$
\operatorname{Pf}\left(\Psi_{A}\right) \operatorname{PT}(+, \rho(B),-)=\sum_{\omega \in S_{n}} \operatorname{PT}(+, \omega(1,2, \ldots, n),-) K_{A}(\omega, \rho(B))
$$

such as to form $(n+2)$-point Parke-Taylor factors. The kinematic factors $K_{A}(\omega, \rho(B))$ are multilinear in the polarization vectors of the set $A$ that enter via $\operatorname{Pf}\left(\Psi_{A}\right)$.

The identical challenge arises at tree level when computing the BCJ master numerators of single-trace $\left(\mathrm{YM}+\phi^{3}\right)$ amplitudes. Recall that both gluons and scalars in $\left(\mathrm{YM}+\phi^{3}\right)$ amplitudes are in the adjoint representation of a color group, and the scalars are additionally in the adjoint representation of a flavor group. A color-stripped amplitude has all the $n$ particles in an ordering, thus the CHY half-integrand is given by a (length- $n$ ) ParkeTaylor factor. In addition, by "single-trace" we mean the scalars are also in an ordering

\footnotetext{
${ }^{10}$ We have included the explicit factor of $\frac{1}{2}$ such that the BCJ numerators $N^{(1)}$ discussed in section 6 directly match those in the literature. It is canceled by parts of the overall normalization factor $\mathscr{N}$ in $(1.1)$ between the amplitude $\mathcal{M}_{L \otimes R}^{\text {tree }}$ and the CHY integral.
} 
after stripping off the flavors, and the other CHY half-integrand is given by a Parke-Taylor factor for scalars in legs $1, \ldots, k$ and a Pfaffian for gluons in legs $k+1, \ldots, n$ [93]. BCJ master numerators are obtained by reducing to Parke-Taylor factors using scattering equations $[26]$ :

$$
\begin{aligned}
& \operatorname{Pf}\left(\Psi_{\{k+1 \ldots n\}}\right) \operatorname{PT}(1, \tau(2, \ldots, k-1), k)=\sum_{\pi \in S_{n-2}} \operatorname{PT}(1, \pi(2, \ldots, k-1, k+1, \ldots, n), k) \\
& \quad \times N_{\mathrm{YM}+\phi^{3}}^{\text {tree }}(1, \pi(2, \ldots, k-1, k+1, \ldots, n), k \mid 1, \tau(2, \ldots, k-1), k)
\end{aligned}
$$

Here without loss of generality, we have chosen the ordering for scalars to be $1, \tau(2, \ldots, k-1), k$ with $\tau \in S_{k-2}$, and the second line of (3.24) can be attained by the techniques of $[35,68,71]$ : the Parke-Taylor coefficients $N_{\mathrm{YM}+\phi^{3}}^{\text {tree }}(\ldots)$ are BCJ master numerators associated with a half-ladder diagram, with 1 and $k$ on opposite ends and the permutations $\pi \in S_{n-2}$ acting on the remaining particles (the second ordering $1, \tau(2, \ldots, k-1), k$ is for the scalars w.r.t. the flavor group).

By matching (3.23) with (3.24), one can identify the kinematic factors $K_{A}(\omega, \rho(B))$ in a one-loop context with a $\left(\mathrm{YM}+\phi^{3}\right)$-master numerator at tree level. One needs to pick the scalars to be in,,$+- B$ and the gluons to be in $A$, and choose the two orderings to match the permutations $\omega, \rho$ :

$$
K_{A}(\omega, \rho(B))=N_{\mathrm{YM}+\phi^{3}}^{\mathrm{tree}}(+, \omega(1,2, \ldots, n),-\mid+, \rho(B),-) .
$$

Two of the current authors present an improved method of computing the necessary $N^{\text {tree }}$ in ref. [72].

As an illustration, let us consider the simplest case with one gluon, i.e. $k=n-1$, then $\operatorname{Pf} \Psi_{\{n\}}=C_{n, n}$ and partial-fraction manipulations are sufficient to show that [68],

$$
\mathrm{C}_{n, n} \operatorname{PT}(1,2, \ldots, n-1)=\sum_{a=1}^{n-2} \mathrm{PT}(1,2, \ldots, a, n, a+1, \ldots, n-1) \sum_{i=1}^{a} \epsilon_{n} \cdot k_{i},
$$

where the ordering for the scalars has been chosen as $1,2, \ldots, n-1$ for simplicity. By (3.25), we have $A=\{n\}$ and $B=\{1,2, \ldots, n-1\}$ for the one-loop case; if we choose $\omega=i d$, then the non-vanishing one-loop master numerators only occur for the ordering $\rho_{a}(B) \equiv$ $(1, \ldots, a, n, a+1, \ldots, n-1)($ for $a=1, \ldots, n-2)$, which $\operatorname{read} K\left(\omega, \rho_{a}(B)\right)=\sum_{i=1}^{a} \epsilon_{n} \cdot k_{i}$.

One can proceed similarly in case of more gluons: for $k=n-2$, by expanding $\operatorname{Pf} \Psi_{\{n-1, n\}}$ and using scattering equation of leg $n$, after some algebra we obtain [68]

$$
\begin{aligned}
\operatorname{Pf} & \Psi_{\{n-1, n\}} \operatorname{PT}(1,2, \ldots, n-2) \\
= & \sum_{a=1}^{n-3} \sum_{b=0}^{n-3-a} \operatorname{PT}(1, \ldots, a, n, \ldots, a+b, n-1, \ldots, n-2)\left(\sum_{i=1}^{a} \epsilon_{n-1} \cdot f_{n} \cdot k_{i}+\sum_{i=1}^{a} \epsilon_{n} \cdot k_{i} \sum_{j=1}^{a+b} \epsilon_{n-1} \cdot k_{j}\right) \\
& +\sum_{a=1}^{n-3} \sum_{b=0}^{n-3-a} \operatorname{PT}(1, \ldots, a, n-1, \ldots, a+b, n, \ldots, n-2) \sum_{i=1}^{a} \epsilon_{n-1} \cdot k_{i}\left(\epsilon_{n} \cdot k_{n-1}+\sum_{j=1}^{a+b} \epsilon_{n} \cdot k_{j}\right),
\end{aligned}
$$


where we have Parke-Taylor factors with label $n$ and $n-1$ inserted at various positions. In this way, one can continue with more and more gluons and obtain BCJ master numerators for single-trace amplitudes in $\mathrm{YM}+\phi^{3}$ [35]. Similar techniques have been used in e.g. [68, $70,71]$, and more recently in $[72,94,95]$.

\section{General gauge theories}

In this section, we move to more general gauge theories in even dimensions whose spectrum may involve an arbitrary combination of adjoint scalars, fermions and gauge bosons. Accordingly, their one-loop correlators are built from forward limits not only in vectors and Weyl fermions but also in scalars. As we will review, the tree-level correlator with 2 scalars and $n-2$ gluons can be obtained from dimension reduction of the $n$-gluon one [38]. By combining all the building blocks from forward limits, we then have a formula for one-loop correlators with $\mathbf{n}_{\mathrm{v}}$ vectors, $\mathbf{n}_{\mathrm{f}}$ Weyl fermions and $\mathbf{n}_{\mathrm{s}}$ scalars in $D$ dimensions. ${ }^{11}$ We will present examples of such correlators in various theories in $D=6$ and $D=4$.

\subsection{Forward limits in general gauge theories}

Before we present a formula for general one-loop correlators in even dimension, let us first review the tree-level correlator involving two scalars. In fact, the bosonic tree-level correlators (2.6) can be straightforwardly adapted to two external scalars in legs $1, n$ by taking their polarizations $\epsilon_{1}, \epsilon_{n}$ to satisfy

$$
\begin{aligned}
& \epsilon_{1} \cdot \epsilon_{n}=1 \\
& \epsilon_{1} \cdot \epsilon_{j}=\epsilon_{n} \cdot \epsilon_{j}=0 \quad \forall \quad j=2,3, \ldots, n-1 \\
& \epsilon_{1} \cdot k_{p}=\epsilon_{n} \cdot k_{p}=0 \quad \forall \quad p=1,2, \ldots, n,
\end{aligned}
$$

which can also be realized from dimensional reduction. The resulting scalar correlator solely features the $B=\emptyset$ term of (2.6),

$$
\mathcal{I}_{2 \mathrm{~s}}^{\text {tree }}\left(1_{\mathrm{s}}, 2, \ldots, n-1, n_{\mathrm{s}}\right)=\operatorname{Pf}\left(\Psi_{\{23 \ldots n-1\}}\right),
$$

where we have used the subscript "s" to denote scalars (recall that gluons have no subscript). The scalar forward limit analogous to (2.20) simply amounts to $\mathrm{FWL}_{i, j}\left(k_{i}, k_{j}\right)=$ $(+\ell,-\ell)$

$$
\mathrm{FWL}_{1, n}\left[\mathcal{I}_{2 \mathrm{~s}}^{\mathrm{tree}}\left(1_{\mathrm{s}}, 2, \ldots, n-1, n_{\mathrm{s}}\right)\right]=\operatorname{Pf}\left(\Psi_{\{23 \ldots n-1\}}\right)
$$

We shall now combine the building blocks (3.1), (3.2) and (4.3) for the forward limits in $D$-dimensional vectors, $D$-dimensional Weyl fermions and scalars. In the presence of $\mathbf{n}_{\mathrm{v}}$, $\mathbf{n}_{\mathrm{f}}$ and $\mathbf{n}_{\mathrm{s}}$ species of vectors, Weyl fermions and scalars, respectively, one arrives at the

\footnotetext{
${ }^{11}$ We denote these numbers of different species by boldface $\mathbf{n}$, to avoid confusion with the $n^{\text {th }}$ external leg.
} 
following parity-even parts of one-loop correlators in even dimensions,

$$
\begin{aligned}
& \mathcal{I}_{\left(\mathbf{n}_{\mathrm{v}}, \mathbf{n}_{\mathrm{f}}, \mathbf{n}_{\mathrm{s}}, D\right)}^{(1)}(1,2, \ldots, n) \\
&= \mathrm{FWL}_{+,-}\left[\mathbf{n}_{\mathrm{v}} \mathcal{I}_{\mathrm{bos}}^{\text {tree }}(+, 1,2, \ldots, n,-)\right. \\
&\left.-\frac{\mathbf{n}_{\mathrm{f}}}{2} \mathcal{I}_{2 \mathrm{f}}^{\text {tree }}\left(+_{\mathrm{f}}, 1,2, \ldots, n,-_{\mathrm{f}}\right)+\mathbf{n}_{\mathrm{s}} \mathcal{I}_{2 \mathrm{~s}}^{\text {tree }}\left(+_{\mathrm{s}}, 1,2, \ldots, n,-_{\mathrm{s}}\right)\right]\left.\right|_{\text {even }} \\
&= \sum_{\substack{\{12 \ldots, n\} \\
=}} \operatorname{Pf}\left(\Psi_{A}\right) \sum_{\rho \in S_{|B|}} \operatorname{PT}(+, \rho(B),-) \\
& \times\left\{\begin{array}{c}
{\left[\mathbf{n}_{\mathrm{v}}(D-2)+\mathbf{n}_{\mathrm{s}}-\mathbf{n}_{\mathrm{f}} 2^{D / 2-2}\right]: B=\emptyset} \\
{\left[\mathbf{n}_{\mathrm{v}} \operatorname{tr}_{\mathrm{V}}\left(\rho\left(b_{1}\right), \rho\left(b_{2}\right), \ldots, \rho\left(b_{|B|}\right)\right)-\frac{\mathbf{n}_{\mathrm{f}}}{2} \operatorname{tr}_{\mathrm{S}}\left(\rho\left(b_{1}\right), \rho\left(b_{2}\right), \ldots, \rho\left(b_{|B|}\right)\right)\right]: B \neq \emptyset}
\end{array} .\right.
\end{aligned}
$$

Note that $\mathbf{n}_{\mathrm{s}}$ only appears in the term with $B=\emptyset$ and $A=\{1,2, \ldots, n\}$, and we again have the loop-momentum dependence $C_{j j}=\epsilon_{j}^{\mu}\left(\frac{\ell_{\mu}}{\sigma_{j,+}}-\frac{\ell_{\mu}}{\sigma_{j,-}}\right)+\ldots$ in $\operatorname{Pf}\left(\Psi_{A}\right)$ for any choice of $A$. Moreover, the coefficient $\mathbf{n}_{\mathrm{v}}(D-2)+\mathbf{n}_{\mathrm{s}}-\mathbf{n}_{\mathrm{f}} 2^{D / 2-2}$ of $\operatorname{Pf}\left(\Psi_{\{12 \ldots n\}}\right)$ can be recognized as the difference of bosonic and fermionic on-shell degrees of freedom: $D$-dimensional vector bosons and Weyl fermions have $D-2$ and $2^{D / 2-2}$ physical degrees of freedom, respectively. Hence, the $B=\emptyset$ contribution to (4.4) is absent in supersymmetric theories.

Given that $\operatorname{tr}_{\mathrm{V}}(\ldots)$ and $\operatorname{tr}_{\mathrm{S}}(\ldots)$ vanish at $|B|=1$, supersymmetric theories admit at most $|A|=n-2$ particles in $\operatorname{Pf}\left(\Psi_{A}\right)$. As a consequence, the maximum power of loop momenta in the parity-even part of supersymmetric correlators is $\ell^{n-2}$, reproducing the power counting of [44] (such power-counting has been studied since the early days of unitarity methods [96-98]). As will be detailed below, the parity-odd contributions to $D=4$ correlators with four supercharges may exceed this bound and involve up to $n-1$ powers of $\ell$.

The $|B|=2$ and $|B|=3$ contributions to (4.4) are proportional to $\mathbf{n}_{\mathrm{v}}-2^{D / 2-5} \mathbf{n}_{\mathrm{f}}$ by the relative factor between vector and spinor traces in (3.8). These terms are absent whenever the ratio of $\mathbf{n}_{\mathrm{v}}$ and $\mathbf{n}_{\mathrm{f}}$ fits to the maximally supersymmetric gauge multiplet in the respective dimension, i.e. $\left(\mathbf{n}_{\mathrm{f}}, \mathbf{n}_{\mathrm{s}}\right)=\left(\mathbf{n}_{\mathrm{v}}, 0\right)$ in $D=10,\left(\mathbf{n}_{\mathrm{f}}, \mathbf{n}_{\mathrm{s}}\right)=\left(4 \mathbf{n}_{\mathrm{v}}, 0\right)$ in $D=6$ and $\left(\mathbf{n}_{\mathrm{f}}, \mathbf{n}_{\mathrm{s}}\right)=\left(8 \mathbf{n}_{\mathrm{v}}, 6 \mathbf{n}_{\mathrm{v}}\right)$ in $D=4$, respectively. Like this, (4.4) manifests that 16 supercharges are a necessary and sufficient condition for the $|B| \leq 3$ contributions to vanish and for the maximum power of loop momentum to be $\ell^{n-4}$.

Finally, the $|B|=4$ contribution to (4.4) is proportional to $\left(\mathbf{n}_{\mathrm{v}}-2^{D / 2-5} \mathbf{n}_{\mathrm{f}}\right) \operatorname{tr}_{\mathrm{V}}(1,2,3,4)+$ $2^{D / 2-6} \mathbf{n}_{\mathrm{f}} t_{8}\left(f_{1}, f_{2}, f_{3}, f_{4}\right)$. In the maximally supersymmetric situation where $\mathbf{n}_{\mathrm{v}}=2^{D / 2-5} \mathbf{n}_{\mathrm{f}}$, the correlator contributions at $|B|=4$ are permutations of $t_{8}\left(f_{1}, f_{2}, f_{3}, f_{4}\right) \operatorname{Pf} \Psi_{\{56 \ldots n\}}$. Hence, the maximally supersymmetric tensor numerators with the highest power of loop momentum $\ell^{n-4}$ are built from a permutation sum over $t_{8}\left(f_{1}, f_{2}, f_{3}, f_{4}\right) \prod_{j=5}^{n}\left(\epsilon_{j} \cdot \ell\right)$.

\subsection{Examples in $D=6$ and $D=4$}

We shall now spell out several examples of the general correlator (4.4) with reduced supersymmetry.

(i) A six-dimensional chiral gauge multiplet with half-maximal supersymmetry (8 supercharges instead of 16) contains a single vector $\mathbf{n}_{\mathrm{v}}=1$ and two Weyl fermions $\mathbf{n}_{\mathrm{f}}=2$ with 
a total of $4+4$ on-shell degrees of freedom

$$
\begin{aligned}
&\left.\mathcal{I}_{(1,2,0, D}^{(1)}=6\right)(1,2, \ldots, n)=\left.\mathrm{FWL}_{+,-}\left[\mathcal{I}_{\text {bos }}^{\text {tree }}(+, 1,2, \ldots, n,-)-\mathcal{I}_{2 \mathrm{f}}^{\text {tree }}\left(+_{\mathrm{f}}, 1,2, \ldots, n,-_{\mathrm{f}}\right)\right]\right|_{\text {even }} ^{D=6} \\
&= \sum_{\substack{\{12 \ldots, \ldots\} \\
=A \cup B}} \operatorname{Pf}\left(\Psi_{A}\right) \sum_{\rho \in S_{|B|}} \operatorname{PT}(+, \rho(B),-) \\
& \quad \times\left\{\begin{array}{cc}
0 & :|B| \leq 1 \\
{\left.\left[\operatorname{tr}_{\mathrm{V}}\left(\rho\left(b_{1}\right), \rho\left(b_{2}\right), \ldots, \rho\left(b_{|B|}\right)\right)-\operatorname{tr}_{\mathrm{S}}\left(\rho\left(b_{1}\right), \rho\left(b_{2}\right), \ldots, \rho\left(b_{|B|}\right)\right)\right]\right|_{D=6}:|B| \geq 2}
\end{array}\right.
\end{aligned} .
$$

The simplest $D=6$ spinor traces resulting from (3.8) and (3.9) include $\operatorname{tr}_{\mathrm{S}}(1,2)=$ $\frac{1}{2} \operatorname{tr}_{\mathrm{V}}(1,2)$ as well as $\operatorname{tr}_{\mathrm{S}}(1,2,3)=\frac{1}{2} \operatorname{tr}_{\mathrm{V}}(1,2,3)$ and introduce the following contributions to $(4.5)$ :

$$
\begin{aligned}
\operatorname{tr}_{\mathrm{V}}(1,2)-\left.\operatorname{tr}_{\mathrm{S}}(1,2)\right|_{D=6}= & \frac{1}{2} \operatorname{tr}_{\mathrm{V}}(1,2) \\
\operatorname{tr}_{\mathrm{V}}(1,2,3)-\left.\operatorname{tr}_{\mathrm{S}}(1,2,3)\right|_{D=6}= & \frac{1}{2} \operatorname{tr}_{\mathrm{V}}(1,2,3) \\
\operatorname{tr}_{\mathrm{V}}(1,2,3,4)-\left.\operatorname{tr}_{\mathrm{S}}(1,2,3,4)\right|_{D=6}= & \frac{1}{4}\left\{3 \operatorname{tr}_{\mathrm{V}}(1,2,3,4)+\operatorname{tr}_{\mathrm{V}}(1,3,2,4)+\operatorname{tr}_{\mathrm{V}}(1,2,4,3)\right\} \\
& -\frac{1}{16}\left\{\operatorname{tr}_{\mathrm{V}}(1,2) \operatorname{tr}_{\mathrm{V}}(3,4)+\operatorname{cyc}(2,3,4)\right\} \\
= & \frac{1}{4} t_{8}\left(f_{1}, f_{2}, f_{3}, f_{4}\right)+\frac{1}{2} \operatorname{tr}_{\mathrm{V}}(1,2,3,4)
\end{aligned}
$$

As a result of the reduced supersymmetry, already the splittings with $|B|=2,3$ contribute to (4.5) which were absent for the ten-dimensional counterpart (3.18) with maximal supersymmetry. Similarly, the six-dimensional combination of four-traces in (4.6) is no longer permutation invariant, i.e.cannot be expressed solely in terms of the $t_{8}$-tensor (3.17).

(ii) A six-dimensional hypermultiplet w.r.t. 8 supercharges contains a single Weyl fermion $\mathbf{n}_{\mathrm{f}}=1$ and two scalars $\mathbf{n}_{\mathrm{s}}=2$ with a total of $2+2$ on-shell degrees of freedom,

$$
\begin{aligned}
& \mathcal{I}_{(0,1,2, D=6)}^{(1)}(1,2, \ldots, n)=\left.\mathrm{FWL}_{+,-}\left[2 \mathcal{I}_{2 \mathrm{~s}}^{\text {tree }}\left(+_{\mathrm{s}}, 1,2, \ldots, n,-_{\mathrm{s}}\right)-\frac{1}{2} \mathcal{I}_{2 \mathrm{f}}^{\text {tree }}\left(+_{\mathrm{f}}, 1,2, \ldots, n,-_{\mathrm{f}}\right)\right]\right|_{\text {even }} ^{D=6} \\
& =-\frac{1}{2} \sum_{\substack{\{12 \ldots n\} \\
=A \cup B}} \operatorname{Pf}\left(\Psi_{A}\right) \sum_{\rho \in S_{|B|}} \operatorname{PT}(+, \rho(B),-)\left\{\begin{array}{cc}
: & |B| \leq 1 \\
\left.\operatorname{tr}_{S}\left(\rho\left(b_{1}\right), \rho\left(b_{2}\right), \ldots, \rho\left(b_{|B|}\right)\right)\right|_{D=6}: & |B| \geq 2
\end{array} .\right.
\end{aligned}
$$

The simplest contributions at $|B|=2,3,4$ are

$$
\begin{aligned}
-\left.\frac{1}{2} \operatorname{tr}_{\mathrm{S}}(1,2)\right|_{D=6} & =-\frac{1}{4} \operatorname{tr}_{\mathrm{V}}(1,2) \\
-\left.\frac{1}{2} \operatorname{tr}_{\mathrm{S}}(1,2,3)\right|_{D=6} & =-\frac{1}{4} \operatorname{tr}_{\mathrm{V}}(1,2,3) \\
-\left.\frac{1}{2} \operatorname{tr}_{\mathrm{S}}(1,2,3,4)\right|_{D=6} & =\frac{1}{8} t_{8}\left(f_{1}, f_{2}, f_{3}, f_{4}\right)-\frac{1}{4} \operatorname{tr}_{\mathrm{V}}(1,2,3,4) .
\end{aligned}
$$

The expressions in (4.6) and (4.8) confirm the decomposition of a ten-dimensional gauge multiplet into one vector multiplet and two hypermultiplets in $D=6$ : by adding two 
copies of (4.8) to (4.6), the two- and three- traces drop out, and one recovers the fourtrace of $D=10$ SYM in (3.16). In section 6.3, we will spell out simplified expressions for $(n \leq 5)$-point BCJ numerators resulting from (4.7) in terms of multiparticle fields.

(iii) Reducing all the way to $D=4$, we can examine a gauge multiplet of $\mathcal{N}=1 \mathrm{SYM}$, which has two fermionic degrees of freedom, so with $\mathbf{n}_{\mathrm{v}}=1$ and $\mathbf{n}_{\mathrm{f}}=2$

$$
\begin{aligned}
\mathcal{I}_{(1,2,0, D=4)}^{(1)}(1,2, \ldots, n)=\left.\mathrm{FWL}_{+,-}\left[\mathcal{I}_{\text {bos }}^{\text {tree }}(+, 1,2, \ldots, n,-)-\mathcal{I}_{2 \mathrm{f}}^{\text {tree }}\left(+_{\mathrm{f}}, 1,2, \ldots, n,--_{\mathrm{f}}\right)\right]\right|_{\text {even }} ^{D=4} \\
=\sum_{\substack{\{12 \ldots, n\} \\
=A \cup B}} \operatorname{Pf}\left(\Psi_{A}\right) \sum_{\rho \in S_{|B|}} \operatorname{PT}(+, \rho(B),-) \\
\quad \times\left\{\begin{array}{cc}
0 & :|B| \leq 1 \\
{\left.\left[\operatorname{tr}_{\mathrm{V}}\left(\rho\left(b_{1}\right), \rho\left(b_{2}\right), \ldots, \rho\left(b_{|B|}\right)\right)-\operatorname{tr}_{\mathrm{S}}\left(\rho\left(b_{1}\right), \rho\left(b_{2}\right), \ldots, \rho\left(b_{|B|}\right)\right)\right]\right|_{D=4}} & :|B| \geq 2
\end{array} .\right.
\end{aligned}
$$

The first three contributions in the $|B| \geq 2$ sector can be easily read off from (3.8) and (3.9), as in the previous examples,

$$
\begin{aligned}
\operatorname{tr}_{\mathrm{V}}(1,2)-\left.\operatorname{tr}_{\mathrm{S}}(1,2)\right|_{D=4}= & \frac{3}{4} \operatorname{tr}_{\mathrm{V}}(1,2) \\
\operatorname{tr}_{\mathrm{V}}(1,2,3)-\left.\operatorname{tr}_{\mathrm{S}}(1,2,3)\right|_{D=4}= & \frac{3}{4} \operatorname{tr}_{\mathrm{V}}(1,2,3) \\
\operatorname{tr}_{\mathrm{V}}(1,2,3,4)-\left.\operatorname{tr}_{\mathrm{S}}(1,2,3,4)\right|_{D=4}= & 2^{-3}\left\{7 \operatorname{tr}_{\mathrm{V}}(1,2,3,4)+\operatorname{tr}_{\mathrm{V}}(1,3,2,4)+\operatorname{tr}_{\mathrm{V}}(1,2,4,3)\right\} \\
& -2^{-5}\left\{\operatorname{tr}_{\mathrm{V}}(1,2) \operatorname{tr}_{\mathrm{V}}(3,4)+\operatorname{cyc}(2,3,4)\right\} \\
= & \frac{1}{8} t_{8}\left(f_{1}, f_{2}, f_{3}, f_{4}\right)+\frac{3}{4} \operatorname{tr}_{\mathrm{V}}(1,2,3,4)
\end{aligned}
$$

Examinations of extended $\mathcal{N}=4,2$ supersymmetry in $D=4$ are redundant since the respective correlators are equivalent to the $D=10$ example in (3.15) and the $D=6$ example in (4.4). In absence of supersymmetry, the four-point instance of (4.4) has been used in [40] to reproduce the BCJ numerators of [99] with up to four powers of loop momentum for the box diagram.

Finally, we remark that the BCJ numerators in these general gauge theories can be extracted from the same worldsheet techniques as for $D=10 \mathrm{SYM}$ : in all cases, their $\sigma$ dependence exclusively enters in the form of $\mathrm{PT}(+, 1,2, \ldots, j,-) \operatorname{Pf} \Psi_{\{j+1 \ldots n\}}$ whose rewriting in terms of $n+2$-point Parke-Taylor factors can be reduced to a solved tree-level problem as discussed in section 3.4. We will present some examples for such BCJ numerators in section 6 and simplify them using multiparticle fields.

\section{$5 \quad$ Parity-odd contributions}

In this section, we derive parity-odd contributions to one-loop correlators from forward limits in chiral fermions. More specifically, this amounts to a parity-odd completion of the correlators (3.15) for $D=10 \mathrm{SYM}$ and those instances of (4.4) with a chiral spectrum. 


\subsection{General prescription and low-multiplicity validation}

The worldsheet prescription for the parity-odd sector of one-loop amplitudes has been discussed in [79, 100, 101] for conventional strings and in [30] for ambitwistor strings. Both approaches have in common that one of the bosonic vertex operators needs to be inserted in the ghost picture -1 . This insertion of $V^{(-1)}$ in $(2.1)$ is essential for zero-mode saturation in the ghost sector and gauge anomalies such as the hexagon anomaly of $D=10$ SYM [102-104].

Accordingly, the forward-limit implementation of the parity-odd sector should start from a tree-level correlator that also has an insertion of $V^{(-1)}$. That is why the forward limit is performed in the representation (2.11) of the two-fermion correlator at tree level, where both two fermions are in the $-1 / 2$ ghost picture. The forward-limit prescription

$$
\left(\chi_{i}\right)^{\alpha}\left(\chi_{j}\right)^{\beta} \rightarrow \mathrm{FWL}_{i, j}\left(\left(\chi_{i}\right)^{\alpha}\left(\chi_{j}\right)^{\beta}\right)=-\ell^{\mu} \gamma_{\mu}^{\alpha \beta}
$$

follows from (2.3) \& (2.21). To ensure the correct relative normalization between the parity-odd and parity-even sectors, we repeat the exercise from sections $3.1 \& 3.3$ of fixing the relative factor $\beta \in \mathbb{Q}$ between the bosonic and fermionic forward limits using known properties,

$$
\begin{aligned}
& \mathcal{I}_{\text {even }, \beta}^{(1)}(\hat{1}, 2,3, \ldots, n)= \\
& \left.\quad \mathrm{FWL}_{+,-}\left[\mathcal{I}_{\text {bos }}^{\text {tree }}(+, 1,2,3, \ldots, n,-)+\beta \mathcal{I}_{2 \mathrm{f}}^{\text {tree }}\left(+_{\mathrm{f}}, \hat{1}, 2,3, \ldots, n,-_{\mathrm{f}}\right)\right]\right|_{\text {even }}
\end{aligned}
$$

With judicious application of scattering equations, the choice $\beta=-1$ reproduces the $n=4$ result calculated in (3.15) \& (3.16). ${ }^{12}$ The forward limit (5.2) has also been studied by Frost [86], where the singularities in $\sigma_{+,-}$were demonstrated to cancel between the bosonic and fermionic contribution. Also, Frost related the fermionic forward limit to the $\tau \rightarrow i \infty$ limit of the Ramond-sector contribution to bosonic one-loop correlators which generalizes the analysis of [65] to ghost pictures $\left(-\frac{1}{2},-\frac{1}{2},-1\right)$.

The parity-odd forward limit inherits this choice of $\beta$, converting the $D$-dimensional version of the tree-level correlator (2.11) into

$$
\begin{aligned}
\mathcal{I}_{\text {odd }}^{(1)}(\hat{1}, 2,3, \ldots, n)= & -\left.\mathrm{FWL}_{+,-}\left[\mathcal{I}_{2 \mathrm{f}}^{\text {tree }}\left(+_{\mathrm{f}}, \hat{1}, 2,3, \ldots, n,-\mathrm{f}\right)\right]\right|_{\text {odd }} \\
= & \frac{1}{2} \sum_{\substack{\{23 \ldots ., n\} \\
=A \cup B \cup C}} \operatorname{Pf}\left(\Psi_{A}\right) \sum_{\rho \in S_{|B|}} \sum_{\tau \in S_{|C|}} \operatorname{PT}(+, \rho(B), n, \tau(C),-) \\
& \times \operatorname{tr}_{\text {odd }}\left(\ell f_{\rho\left(b_{1}\right)} f_{\rho\left(b_{2}\right)} \ldots f_{\rho\left(b_{|B|}\right)} \notin_{1} f_{\tau\left(c_{1}\right)} f_{\tau\left(c_{2}\right)} \ldots f_{\tau\left(c_{|C|}\right)}\right) .
\end{aligned}
$$

\footnotetext{
${ }^{12}$ Also, one could in principle compute the parity-even sector of higher-point correlators using this forward limit. However, the presence of $\ell$ and $\phi_{1}$ (without an accompanying $\not k_{1}$ ) obscure the supersymmetry cancelations, requiring increasingly complicated application of scattering equations. As we will see shortly, the choice for $\beta$ is also reinforced by matching the expected relative factor between parity-odd and parity-even results.
} 
The notation $\operatorname{tr}_{\text {odd }}(\ldots)$ instructs to only keep the parity-odd part of the chiral trace ${ }^{13}$ proportional to the Levi-Civita symbol $\varepsilon^{\mu_{1} \mu_{2} \ldots \mu_{D}}$,

$$
\left.\operatorname{tr}_{\text {odd }}\left(\ell f_{i} \ldots f_{j} \notin_{1} f_{p} \ldots f_{q}\right) \equiv \ell^{\mu} \gamma_{\mu}^{\alpha \beta}\left(f_{i} \ldots f_{j} \phi_{1} f_{p} \ldots f_{q}\right)_{\alpha \beta}\right|_{\text {odd }} .
$$

Accordingly, $\operatorname{tr}_{\text {odd }}(\ldots)$ with less than $D$ gamma matrices in the ellipsis automatically vanish,

$$
\operatorname{tr}_{\text {odd }}\left(\gamma^{\mu_{1}} \gamma^{\mu_{2}} \ldots \gamma^{\mu_{p}}\right)=0 \quad \forall p<D .
$$

Hence, the partitions of $\{2,3, \ldots, n\}$ into $A, B, C$ must have $|B|+|C| \geq \frac{D}{2}-1$ in order to allow for a non-vanishing trace, starting with

$$
\operatorname{tr}_{\text {odd }}\left(\gamma^{\mu_{1}} \gamma^{\mu_{2}} \ldots \gamma^{\mu_{D}}\right)=i 2^{D / 2-1} \varepsilon^{\mu_{1} \mu_{2} \ldots \mu_{D}} .
$$

This implies a minimum multiplicity $n=\frac{D}{2}$ to obtain non-zero parity-odd correlators (5.3)

$$
\begin{aligned}
\left.\mathcal{I}_{\text {odd }}^{(1)}(\hat{1}, 2, \ldots, n)\right|_{n<D / 2}= & 0 \\
\left.\mathcal{I}_{\text {odd }}^{(1)}(\hat{1}, 2, \ldots, n)\right|_{n=D / 2}= & \frac{1}{2} \sum_{\substack{\{23 \ldots D / 2\} \\
=B \cup C}} \sum_{\rho \in S_{|B|}} \sum_{\tau \in S_{|C|}} \mathrm{PT}(+, \rho(B), n, \tau(C),-) \\
& \times \operatorname{tr}_{\text {odd }}\left(\ell f_{\rho\left(b_{1}\right)} \ldots f_{\rho\left(b_{|B|}\right)} \phi_{1} f_{\tau\left(c_{1}\right)} \ldots f_{\tau\left(c_{|C|}\right)}\right) \\
= & 2^{-D / 2} i \varepsilon_{\mu_{1} \mu_{2} \ldots \mu_{D}} \ell^{\mu_{1}} \epsilon_{1}^{\mu_{2}} f_{2}^{\mu_{3} \mu_{4}} f_{3}^{\mu_{5} \mu_{6}} \ldots f_{D / 2}^{\mu_{D-1} \mu_{D}} \\
& \times \sum_{\rho \in S_{D / 2}} \mathrm{PT}\left(+, \rho\left(1,2, \ldots, \frac{D}{2}\right),-\right),
\end{aligned}
$$

in lines with the analysis of fermionic zero mode in one-loop worldsheet prescriptions [30, $79,100,101]$. Moreover, the tensor structure of the $\left(\frac{D}{2}\right)$-point correlator (5.8) is entirely determined by the fermionic zero modes. Like this, the permutation-symmetric sum over Parke-Taylor factors in (5.8) is consistent with the worldsheet derivation. In order to avoid proliferation of indices, we employ shorthands

$$
\varepsilon_{D}\left(v_{1}, v_{2}, \ldots, v_{D}\right)=\varepsilon_{\mu_{1} \mu_{2} \ldots \mu_{D}} v_{1}^{\mu_{1}} v_{2}^{\mu_{2}} \ldots v_{D}^{\mu_{D}}, \quad \varepsilon_{D}^{\mu}\left(v_{2}, \ldots, v_{D}\right)=\varepsilon_{\lambda_{2} \ldots \lambda_{D}}^{\mu} v_{2}^{\lambda_{2}} \ldots v_{D}^{\lambda_{D}}
$$

for Levi-Civita contractions of $D$-dimensional vectors $v_{j}$. In this notation, the permutationsymmetric BCJ-numerators following from (5.8) are given by

$$
N_{\text {odd }}^{(1)}\left(+, \rho\left(1,2, \ldots, \frac{D}{2}\right),-\right)=i \varepsilon_{D}\left(\ell, \epsilon_{1}, k_{2}, \epsilon_{2}, k_{3}, \epsilon_{3}, \ldots, k_{D / 2}, \epsilon_{D / 2}\right)
$$

after absorbing the leading factor of $\frac{1}{2}$ following the definition of $N^{(1)}$ from (3.22).

In $D=10$ dimensions, this becomes a five-point numerator that reproduces the parityodd part of the pentagon numerator $i \varepsilon_{10}\left(\ell, \epsilon_{1}, k_{2}, \epsilon_{2}, k_{3}, \epsilon_{3}, \ldots, k_{5}, \epsilon_{5}\right)$ in ten-dimensional

\footnotetext{
${ }^{13}$ When contracting $\ell^{\mu}, f_{i}^{\mu \nu}$ and $\epsilon_{1}^{\mu}$ with $2^{D / 2} \times 2^{D / 2}$ Dirac gamma matrices $\Gamma^{\mu}$ instead of the $2^{D / 2-1} \times$ $2^{D / 2-1}$ Weyl blocks $\gamma^{\mu}$, one can obtain $\operatorname{tr}_{\text {odd }}(\ldots)$ by inserting the $D$-dimensional chirality matrix $\Gamma_{D+1}$ into the trace.
} 
SYM $[15,44]$. With the normalization of (5.10) and (3.15), we arrive at the relative factor of parity-even and -odd terms known from [105] that plays an important role for S-duality of the five-point one-loop amplitude of type-IIB superstrings. Similarly, (5.10) in $D=6$ yields the parity-odd term $i \varepsilon_{6}\left(\ell, \epsilon_{1}, k_{2}, \epsilon_{2}, k_{3}, \epsilon_{3}\right)$ in the triangle numerator of chiral sixdimensional SYM with eight supercharges $[44,106]$.

\subsection{Anomalies and their singled-out leg}

In order to reproduce the expected gauge anomalies from our parity-odd correlators, we need to evaluate the forward-limit prescription (5.3) at multiplicities $\geq \frac{D}{2}+1$. This requires chiral gamma traces beyond (5.5) and (5.6) such as ${ }^{14}$

$$
\operatorname{tr}_{\text {odd }}\left(\gamma^{\mu_{1}} \gamma^{\mu_{2}} \ldots \gamma^{\mu_{D+2}}\right)=-i 2^{D / 2-1} \sum_{2 \leq i<j \leq D+2}(-1)^{(j-i)} \eta^{\mu_{i} \mu_{j}} \varepsilon^{\mu_{1} \ldots \tilde{\mu}_{i} \ldots \tilde{\mu}_{j} \ldots \mu_{D+2}}
$$

and its generalizations, details of which are provided in appendix C.3 (also see (4.35b) of [65] for an alternative form of the all-multiplicity result). We have checked for the sixpoint correlator of $D=10 \mathrm{SYM}$ and for the four-point correlator of chiral SYM in $D=6$ that the forward-limit prescription (5.3) reproduces the expressions of [44],

$$
\begin{aligned}
\left.\mathcal{I}_{\text {odd }}^{(1)}(\hat{1}, 2, \ldots, n)\right|_{n=D / 2+1}=i \ell_{\mu} \sum_{\rho \in S_{D / 2+1}} \operatorname{PT}\left(+, \rho\left(1,2, \ldots, \frac{D}{2}+1\right),-\right) \\
\times\left\{\left[\left(\ell \cdot \epsilon_{2}\right) \varepsilon_{D}^{\mu}\left(\epsilon_{1}, k_{3}, \epsilon_{3}, \ldots, k_{D / 2+1}, \epsilon_{D / 2+1}\right)+\left(2 \leftrightarrow 3,4, \ldots, \frac{D}{2}+1\right)\right]\right. \\
+\frac{1}{2}\left[\operatorname{sgn}_{23}^{\rho} E_{1 \mid 23,4, \ldots, D / 2+1}^{\mu}+\left(2,3 \mid 2,3,4, \ldots, \frac{D}{2}+1\right)\right] \\
\left.+\frac{1}{2}\left[\operatorname{sgn}_{12}^{\rho} E_{12 \mid 3,4, \ldots, D / 2+1}^{\mu}+\left(2 \leftrightarrow 3,4, \ldots, \frac{D}{2}+1\right)\right]\right\},
\end{aligned}
$$

where the notation $\left(2,3 \mid 2,3,4, \ldots, \frac{D}{2}+1\right)$ is explained below (3.21). The $\rho$-dependent signs $\operatorname{sgn}_{i j}^{\rho}$ are defined in (3.13), and we have introduced the following shorthands for the tensor structures in the last two lines:

$$
\begin{aligned}
E_{12 \mid 3,4, \ldots, p}^{\mu}= & \left(\epsilon_{1} \cdot k_{2}\right) \varepsilon_{D}^{\mu}\left(\epsilon_{2}, k_{3}, \epsilon_{3}, \ldots, k_{p}, \epsilon_{p}\right)-\left(\epsilon_{2} \cdot k_{1}\right) \varepsilon_{D}^{\mu}\left(\epsilon_{1}, k_{3}, \epsilon_{3}, \ldots, k_{p}, \epsilon_{p}\right) \\
& -\left(\epsilon_{1} \cdot \epsilon_{2}\right) \varepsilon_{D}^{\mu}\left(k_{2}, k_{3}, \epsilon_{3}, \ldots, k_{p}, \epsilon_{p}\right) \\
E_{1 \mid 23,4, \ldots, p}^{\mu}= & \left(\epsilon_{2} \cdot k_{3}\right) \varepsilon_{D}^{\mu}\left(\epsilon_{1}, k_{2}+k_{3}, \epsilon_{3}, \ldots, k_{p}, \epsilon_{p}\right)-\left(\epsilon_{3} \cdot k_{2}\right) \varepsilon_{D}^{\mu}\left(\epsilon_{1}, k_{23}, \epsilon_{2}, \ldots, k_{p}, \epsilon_{p}\right) \\
& -\left(\epsilon_{2} \cdot \epsilon_{3}\right) \varepsilon_{D}^{\mu}\left(\epsilon_{1}, k_{2}, k_{3}, \ldots, k_{p}, \epsilon_{p}\right)-\left(k_{2} \cdot k_{3}\right) \varepsilon_{D}^{\mu}\left(\epsilon_{1}, \epsilon_{2}, \epsilon_{3}, \ldots, k_{p}, \epsilon_{p}\right) .
\end{aligned}
$$

\footnotetext{
${ }^{14}$ We use $\tilde{\mu}_{j}$ to denote that $\mu_{j}$ is missing from the index list. The standard convention is to use $\hat{\mu}_{j}$, but we wish to avoid confusion with the special fermion leg which we have also labeled with $\hat{i}$. Additionally, it is worth noting that it is possible to use overantisymmetrization identity from (5.16) to rewrite (5.12) in a more symmetric form and to extend the summation range to all of $1 \leq i<j \leq D+2$,

$$
\operatorname{tr}_{\text {odd }}\left(\gamma^{\mu_{1}} \gamma^{\mu_{2}} \ldots \gamma^{\mu_{D+2}}\right)=-i 2^{D / 2-1} \sum_{1 \leq i<j \leq D+2}(-1)^{(j-i)} \eta^{\mu_{i} \mu_{j}} \varepsilon^{\mu_{1} \ldots \tilde{\mu}_{i} \ldots \tilde{\mu}_{j} \ldots \mu_{D+2}}
$$

More generally, the overantisymmetrization identity can be used to remove any specific index label from the summation range, freezing it to only appear in the $\varepsilon$ tensor.
} 
Note that we have used the overantisymmetrization identity

$$
\varepsilon^{\left[\mu_{1} \mu_{2} \ldots \mu_{D}\right.} \eta^{\left.\mu_{D+1}\right] \lambda}=0
$$

in deriving (5.13) from (5.3). As a major advantage of the forward-limit prescription (5.3), it bypasses the reference to the spurious position of the picture-changing operator in the one-loop worldsheet prescription [30]. Like this, the Parke-Taylor decomposition of ( $n \geq$ $\left.\frac{D}{2}+1\right)$-point correlators is greatly facilitated by the approach in this section.

On the support of the scattering equations, (5.13) vanishes under linearized gauge variations $\epsilon_{j} \rightarrow k_{j}$ in all the legs $j=2,3, \ldots, n$ except for the first one. The variation $\epsilon_{1} \rightarrow k_{1}$ in the leg which is singled out by the hat notation in (5.13) is proportional to $\ell^{2}$

$$
\left.\mathcal{I}_{\text {odd }}^{(1)}(\hat{1}, 2, \ldots, n)\right|_{n=D / 2+1} ^{\epsilon_{1} \rightarrow k_{1}}=i \ell^{2} \varepsilon_{D}\left(k_{2}, \epsilon_{2}, k_{3}, \epsilon_{3}, \ldots, k_{D / 2+1}, \epsilon_{D / 2+1}\right)
$$

and therefore yields rational loop integrals, see section 5.5 of [44] for details in a CHY context. $^{15}$

Given the asymmetric gauge variations, the $\left(\frac{D}{2}+1\right)$-point correlator (5.13) cannot be permutation invariant, not even on the support of scattering equations. Indeed, the difference between singling out legs 1 and 2 through the ghost picture $(-1)$ in $(2.11)$ is given by [44]

$$
\begin{aligned}
& \mathcal{I}_{\text {odd }}^{(1)}(\hat{1}, 2,3, \ldots, n)-\left.\mathcal{I}_{\text {odd }}^{(1)}(1, \hat{2}, 3, \ldots, n)\right|_{n=D / 2+1} \\
& \quad=-\ell^{2} \varepsilon_{D}\left(\epsilon_{1}, \epsilon_{2}, k_{3}, \epsilon_{3}, k_{4}, \epsilon_{4}, \ldots, k_{D / 2+1}, \epsilon_{D / 2+1}\right) \sum_{\rho \in S_{D / 2+1}} \mathrm{PT}\left(+, \rho\left(1,2, \ldots, \frac{D}{2}+1\right),-\right),
\end{aligned}
$$

see [62] for the analogous asymmetry of the one-loop six-point amplitude of the pure-spinor superstring.

\section{BCJ numerators in terms of multiparticle fields}

In this section, we provide alternative representations of the BCJ numerators, where the contributions from the Pfaffian and the field-strength traces in the correlators (3.15), (4.4) and (5.3) are combined. The driving force for particularly compact expressions are socalled multiparticle fields - essentially the numerators of Berends-Giele currents [74] in BCJ gauge, where the color-kinematics duality is manifest [60, 75]. Multiparticle fields were initially constructed in pure-spinor superspace [73] (see [13, 14] for precursors in the context of superstring tree amplitudes) and later on formulated in components for arbitrary combinations of bosons and fermions [61]. They became central ingredients of BCJ numerators $[13,15,17,44]$ and correlators for multiparticle string amplitudes $[58,62-$ 64, 108].

\footnotetext{
${ }^{15}$ See $[15,107]$ for earlier discussions in a field-theory context and $[58,62,100]$ in a string-theory context.
} 


\subsection{Brief review}

Multiparticle polarizations $\epsilon_{P}^{\mu}$ and field strengths $f_{P}^{\mu \nu}$ will be indexed by words $P=12 \ldots p$ or multiparticle labels. This subsection simply collects the definitions relevant to later equations, and the reader is referred to $[61,106,109]$ for further background.

Two-particle versions of polarization vectors and field strengths are defined by

$$
\begin{aligned}
\epsilon_{12}^{\mu} & =\left(k_{2} \cdot \epsilon_{1}\right) \epsilon_{2}^{\mu}-\left(k_{1} \cdot \epsilon_{2}\right) \epsilon_{1}^{\mu}+\frac{1}{2}\left(\epsilon_{1} \cdot \epsilon_{2}\right)\left(k_{1}^{\mu}-k_{2}^{\mu}\right) \\
& =\frac{1}{2}\left\{\left(k_{2} \cdot \epsilon_{1}\right) \epsilon_{2}^{\mu}+\left(\epsilon_{1}\right)_{\nu} f_{2}^{\nu \mu}-(1 \leftrightarrow 2)\right\} \\
f_{12}^{\mu \nu} & =\left(k_{2} \cdot \epsilon_{1}\right) f_{2}^{\mu \nu}-\left(k_{1} \cdot \epsilon_{2}\right) f_{1}^{\mu \nu}+f_{1 \lambda}^{\mu} f_{2}^{\lambda \nu}-f_{2 \lambda}^{\mu} f_{1}^{\lambda \nu} \\
& =k_{12}^{\mu} \epsilon_{12}^{\nu}-k_{12}^{\nu} \epsilon_{12}^{\mu}-\left(k_{1} \cdot k_{2}\right)\left(\epsilon_{1}^{\mu} \epsilon_{2}^{\nu}-\epsilon_{1}^{\nu} \epsilon_{2}^{\mu}\right)
\end{aligned}
$$

and obey $\epsilon_{12}^{\mu}=-\epsilon_{21}^{\mu}$ as well as $f_{12}^{\mu \nu}=-f_{21}^{\mu \nu}$. Here and below, the notation for multiparticle momenta is

$$
k_{12 \ldots p}=k_{1}+k_{2}+\ldots+k_{p} .
$$

Three-particle polarizations are defined in two steps: promoting (6.1) to a recursion with labels $(1,2) \rightarrow(12,3)$ yields the intermediate expression

$$
\widehat{\epsilon}_{123}^{\mu}=\frac{1}{2}\left\{\left(k_{3} \cdot \epsilon_{12}\right) \epsilon_{3}^{\mu}-\left(k_{12} \cdot \epsilon_{3}\right) \epsilon_{12}^{\mu}+\left(\epsilon_{12}\right)_{\nu} f_{3}^{\nu \mu}-\left(\epsilon_{3}\right)_{\nu} f_{12}^{\nu \mu}\right\}
$$

subject to $\widehat{\epsilon}_{123}^{\mu}=-\widehat{\epsilon}_{213}^{\mu}$. Given that $\widehat{\epsilon}_{123}^{\mu}+\widehat{\epsilon}_{231}^{\mu}+\widehat{\epsilon}_{312}^{\mu}=3 k_{123}^{\mu} h_{123}$ for some scalar $h_{123}$ given below, an improved version that obeys the kinematic off-shell Jacobi identity $\epsilon_{123}^{\mu}+\epsilon_{231}^{\mu}+$ $\epsilon_{312}^{\mu}=0$ on top of $\epsilon_{123}^{\mu}=-\epsilon_{213}^{\mu}$ follows from the redefinition

$$
\epsilon_{123}^{\mu}=\widehat{\epsilon}_{123}^{\mu}-k_{123}^{\mu} h_{123}, \quad h_{123}=\frac{1}{12} \epsilon_{1}^{\mu}\left(f_{2}\right)_{\mu \nu} \epsilon_{3}^{\nu}+\operatorname{cyc}(1,2,3)=-h_{213}=-h_{132},
$$

which is part of a non-linear gauge transformation [60]. The associated field strength subject to $f_{123}^{\mu \nu}=-f_{213}^{\mu \nu}$ and $f_{123}^{\mu \nu}+f_{231}^{\mu \nu}+f_{312}^{\mu \nu}=0$ is

$$
f_{123}^{\mu \nu}=k_{123}^{\mu} \epsilon_{123}^{\nu}-\left(k_{12} \cdot k_{3}\right) \epsilon_{12}^{\mu} \epsilon_{3}^{\nu}-\left(k_{1} \cdot k_{2}\right)\left(\epsilon_{1}^{\mu} \epsilon_{23}^{\nu}-\epsilon_{2}^{\mu} \epsilon_{13}^{\nu}\right)-(\mu \leftrightarrow \nu),
$$

where $h_{123}$ drops out from $k_{123}^{\mu} \epsilon_{123}^{\nu}-(\mu \leftrightarrow \nu)$. Some of the later numerators involve the four-particle field strength that can be assembled from

$$
\begin{aligned}
\widehat{\epsilon}_{1234}^{\mu}= & \frac{1}{2}\left\{\left(k_{4} \cdot \epsilon_{123}\right) \epsilon_{4}^{\mu}-\left(k_{123} \cdot \epsilon_{4}\right) \epsilon_{123}^{\mu}+\left(\epsilon_{123}\right)_{\nu} f_{4}^{\nu \mu}-\left(\epsilon_{4}\right)_{\nu} f_{123}^{\nu \mu}\right\} \\
\epsilon_{1234}^{\prime \mu}= & \widehat{\epsilon}_{1234}^{\mu}-\left(k_{12} \cdot k_{3}\right) \epsilon_{3}^{\mu} h_{124}-\left(k_{1} \cdot k_{2}\right)\left(\epsilon_{2}^{\mu} h_{134}-\epsilon_{1}^{\mu} h_{234}\right) \\
f_{1234}^{\mu \nu}= & k_{1234}^{\mu} \epsilon_{1234}^{\prime \nu}-\left(k_{123} \cdot k_{4}\right) \epsilon_{123}^{\mu} \epsilon_{4}^{\nu}-\left(k_{12} \cdot k_{3}\right)\left(\epsilon_{12}^{\mu} \epsilon_{34}^{\nu}+\epsilon_{124}^{\mu} \epsilon_{3}^{\nu}\right) \\
& -\left(k_{1} \cdot k_{2}\right)\left(\epsilon_{13}^{\mu} \epsilon_{24}^{\nu}+\epsilon_{14}^{\mu} \epsilon_{23}^{\nu}+\epsilon_{134}^{\mu} \epsilon_{2}^{\nu}-\epsilon_{234}^{\mu} \epsilon_{1}^{\nu}\right)-(\mu \leftrightarrow \nu)
\end{aligned}
$$

and obeys $f_{1234}^{\mu \nu}=-f_{2134}^{\mu \nu}$ and $f_{1234}^{\mu \nu}+f_{2314}^{\mu \nu}+f_{3124}^{\mu \nu}=0$ as well as $f_{1234}^{\mu \nu}-f_{1243}^{\mu \nu}+f_{3412}^{\mu \nu}-f_{3421}^{\mu \nu}=0$. 


\section{2 $D=10$ examples}

We shall now express $(n \geq 5)$-point examples of the $D=10$ SYM correlators (3.15) in terms of multiparticle fields and provide a new seven-point result. At six points, we spell out local versions of the manifestly gauge-invariant BCJ numerators in [44], also see [15] for their supersymmetrization. In the same way as the $t_{8}$-tensor (3.17) furnishes the four-point BCJ numerators in (3.18), higher-point numerators will boil down to its contraction with multiparticle field strengths such as (6.2), (6.6) and (6.7),

$$
t_{8}(A, B, C, D)=t_{8}\left(f_{A}, f_{B}, f_{C}, f_{D}\right) .
$$

The symmetries of $f_{A}^{\mu \nu}$ in its multiparticle label $A=12 \ldots$ propagate to $(6.8)$ in the obvious manner, e.g. $f_{12}^{\mu \nu}=-f_{21}^{\mu \nu}$ and $f_{123}^{\mu \nu}+\operatorname{cyc}(1,2,3)=0$ imply that $t_{8}(12,3,4,5)=$ $-t_{8}(21,3,4,5)$ and $t_{8}(123,4,5,6)+\operatorname{cyc}(1,2,3)=0$, respectively. We also introduce vectorial generalizations

$$
\begin{aligned}
t_{8}^{\mu}(A, B, C, D, E)= & \epsilon_{A}^{\mu} t_{8}(B, C, D, E)+\epsilon_{B}^{\mu} t_{8}(A, C, D, E)+\epsilon_{C}^{\mu} t_{8}(A, B, D, E) \\
& +\epsilon_{D}^{\mu} t_{8}(A, B, C, E)+\epsilon_{E}^{\mu} t_{8}(A, B, C, D)
\end{aligned}
$$

and tensorial ones

$$
\begin{aligned}
t_{8}^{\mu \nu}(A, B, \ldots, F) & =\left(\epsilon_{A}^{\mu} \epsilon_{B}^{\nu}+\epsilon_{A}^{\nu} \epsilon_{B}^{\mu}\right) t_{8}(C, D, E, F)+(A, B \mid A, B, \ldots, F) \\
t_{8}^{\mu \nu \lambda}(A, B, \ldots, G) & =\left(\epsilon_{A}^{\mu} \epsilon_{B}^{\nu} \epsilon_{C}^{\lambda}+\operatorname{symm}(\mu, \nu, \lambda)\right) t_{8}(D, E, F, G)+(A, B, C \mid A, B, \ldots, G) .
\end{aligned}
$$

These building blocks are symmetric under exchange of multiparticle labels, say $t_{\ddot{8}}(A, B, \ldots)$ $=t_{\ddot{8}}(B, A, \ldots)$, obey the symmetries of $\epsilon_{A}^{\mu}, f_{B}^{\mu \nu}$ within $A, B, \ldots$ and follow the combinatorics of their counterparts in pure-spinor superspace $[63,110]$.

From the decomposition (cf. (3.22))

$$
\mathcal{I}_{D=10 \mathrm{SYM}}^{(1)}(1,2, \ldots, n)=\frac{1}{2} \sum_{\rho \in S_{n}} N_{\max }^{(1)}(+, \rho(1,2, \ldots, n),-) \mathrm{PT}(+, \rho(1,2, \ldots, n),-),
$$

we find the four- and five-point numerators

$$
\begin{aligned}
N_{\max }^{(1)}(+, 1,2,3,4,-)= & t_{8}(1,2,3,4) \\
N_{\max }^{(1)}(+, 1,2,3,4,5,-)= & \ell_{\mu} t_{8}^{\mu}(1,2,3,4,5)-\frac{1}{2}\left\{t_{8}(12,3,4,5)+t_{8}(13,2,4,5)\right. \\
& +t_{8}(14,2,3,5)+t_{8}(15,2,3,4)+t_{8}(23,1,4,5)+t_{8}(24,1,3,5) \\
& \left.+t_{8}(25,1,3,4)+t_{8}(34,1,2,5)+t_{8}(35,1,2,4)+t_{8}(45,1,2,3)\right\} .
\end{aligned}
$$

Note that the contribution $f_{1 \lambda}^{\mu} f_{2}^{\lambda \nu}-f_{2 \lambda}^{\mu} f_{1}^{\lambda \nu}$ to the two-particle field strength in (6.2) stems from the commutators $\left[f_{1}, f_{2}\right]^{\mu \nu}$ in the $t_{8}$-representation of the five-traces (3.19). The remaining contributions to $f_{12}^{\mu \nu}$ such as $\left(k_{2} \cdot \epsilon_{1}\right) f_{2}^{\mu \nu}-\left(k_{1} \cdot \epsilon_{2}\right) f_{1}^{\mu \nu}$ are due to $\operatorname{Pf}\left(\Psi_{\{1\}}\right)\left[\operatorname{tr}_{\mathrm{V}}\left(f_{2} f_{3} f_{4} f_{5}\right)-\frac{1}{2} \operatorname{tr}_{\mathrm{S}}\left(f_{2} f_{3} f_{4} f_{5}\right)\right]$ and its permutations in (3.15). Supersymmetric BCJ numerator on quadratic propagators with the structure of (6.12) have been constructed in [15]. Moreover, the $t_{8}$-tensors in (6.12) have been later on derived from the 
one-loop ambitwistor-string prescription in the RNS formalism, see appendix D of [44]. Additionally, antisymmetrizing (6.12) in 1,2, we find the numerator of a massive box diagram (with legs 1,2 in a dangling tree) to be $t_{8}(12,3,4,5)$.

On the support of scattering equations, the six-point analogues of (6.12) following from the correlators (3.15) are

$$
\begin{aligned}
& N_{\max }^{(1)}(+, 1,2,3,4,5,6,-)=\frac{1}{2} \ell_{\mu} \ell_{\nu} t_{8}^{\mu \nu}(1,2,3,4,5,6)-\frac{1}{2} \ell_{\mu}\left\{t_{8}^{\mu}(12,3,4,5,6)+(1,2 \mid 1,2, \ldots, 6)\right\} \\
& +\frac{1}{4}\left\{t_{8}(12,34,5,6)+t_{8}(13,24,5,6)+t_{8}(14,23,5,6)+(5,6 \mid 1,2, \ldots, 6)\right\} \\
& +\frac{1}{6}\left\{t_{8}(123,4,5,6)+t_{8}(321,4,5,6)+(1,2,3 \mid 1,2, \ldots, 6)\right\}+\widehat{t}_{12}(1,2,3,4,5,6) .
\end{aligned}
$$

The linear order in loop momentum follows the combinatorics of the $\ell$-independent fivepoint numerator in (6.12), i.e. with all $t_{8}^{\mu}(i j, \ldots)$ subject to $1 \leq i<j \leq 6$. The $\ell$ independent part features a total of 45 arrangements $t_{8}(i j, p q, \ldots)$ with $1 \leq i<j \leq 6,1 \leq p<q \leq 6$ and $i<p$ as well as 20 pairs of terms $t_{8}(i j k, \ldots)+t_{8}(k j i, \ldots)$ with $1 \leq i<j<k \leq 6$. Finally, the last term of (6.13) adds a permutation symmetric piece to the zeroth order in $\ell$ :

$$
\begin{aligned}
\widehat{t}_{12}(1,2,3,4,5,6)= & \frac{1}{12}\left\{\left(k_{2}^{\mu}-k_{1}^{\mu}\right) t_{8}^{\mu}(12,3,4,5,6)+(1,2 \mid 1,2,3,4,5,6)\right\} \\
& -\frac{1}{24} t_{8}^{\mu \nu}(1,2,3,4,5,6) \sum_{j=1}^{6} k_{j}^{\mu} k_{j}^{\nu} .
\end{aligned}
$$

Note that the combinatorics of (6.13) and (6.14) also governs the supersymmetric and local hexagon numerator in (4.35) of [15]. As indicated by the widehat of $\widehat{t}_{12},(6.14)$ is not the gauge-invariant $t_{12}$-quantity in (3.20).

In the seven-point generalization of (6.13), all $\ell$-dependent terms can be anticipated by adjoining a vector index to the building blocks of the above $N_{\max }^{(1)}(+, 1, \ldots, 6,-)$, see the first three lines of

$$
\begin{aligned}
& N_{\max }^{(1)}(+, 1,2, \ldots, 7,-)=\frac{1}{6} \ell_{\mu} \ell_{\nu} \ell_{\lambda} t_{8}^{\mu \nu \lambda}(1,2, \ldots, 7)-\frac{1}{4} \ell_{\mu} \ell_{\nu}\left\{t_{8}^{\mu \nu}(12,3, \ldots, 7)+(1,2 \mid 1,2, \ldots, 7)\right\} \\
& +\frac{1}{4} \ell_{\mu}\left\{t_{8}^{\mu}(12,34,5,6,7)+t_{8}^{\mu}(13,24,5,6,7)+t_{8}^{\mu}(14,23,5,6,7)+(5,6,7 \mid 1,2, \ldots, 7)\right\} \\
& +\frac{1}{6} \ell_{\mu}\left\{t_{8}^{\mu}(123,4, \ldots, 7)+t_{8}^{\mu}(321,4, \ldots, 7)+(1,2,3 \mid 1,2, \ldots, 7)\right\}+\ell_{\mu} \widehat{t}_{12}^{\mu}(1,2,3, \ldots, 7) \\
& -\frac{1}{2}\left\{\widehat{t}_{12}(12,3, \ldots, 7)+(1,2 \mid 1,2, \ldots, 7)\right\}-\frac{1}{8}\left\{t_{8}(12,34,56,7)+104 \text { others }\right\} \\
& -\frac{1}{12}\left\{t_{8}(123,45,6,7)+t_{8}(321,45,6,7)+209 \text { other pairs }\right\} \\
& +\frac{1}{12}\left\{-t_{8}(1234,5,6,7)+t_{8}(4321,5,6,7)+t_{8}(1423,5,6,7)\right. \\
& \left.\quad+t_{8}(2314,5,6,7)+(5,6,7 \mid 1,2, \ldots, 7)\right\} \\
& -\frac{1}{96}\left\{\Delta_{8}(12 \mid 3,4,5,6,7)+(1,2 \mid 1,2, \ldots, 7)\right\} .
\end{aligned}
$$


The $\ell$-independent terms in the last five lines contain the new seven-point information. ${ }^{16}$ We have introduced a vectorial and a two-particle version of the permutation symmetric hexagon building block (6.14),

$$
\begin{aligned}
\widehat{t}_{12}^{\mu}(1,2,3, \ldots, 7)= & \frac{1}{12}\left\{\left(k_{2}^{\lambda}-k_{1}^{\lambda}\right) t_{8}^{\mu \lambda}(12,3, \ldots, 7)+(1,2 \mid 1,2, \ldots, 7)\right\} \\
& -\frac{1}{24} t_{8}^{\mu \nu \lambda}(1,2, \ldots, 7) \sum_{j=1}^{7} k_{j}^{\nu} k_{j}^{\lambda} \\
\widehat{t}_{12}(12,3, \ldots, 7)= & \frac{1}{12}\left\{\left(k_{3}^{\mu}-k_{12}^{\mu}\right) t_{8}^{\mu}(123,4,5,6,7)+(3 \leftrightarrow 4,5,6,7)\right\} \\
& +\frac{1}{12}\left\{\left(k_{4}^{\mu}-k_{3}^{\mu}\right) t_{8}^{\mu}(12,34,5,6,7)+(3,4 \mid 3,4,5,6,7)\right\} \\
& -\frac{1}{24} t_{8}^{\mu \nu}(12,3, \ldots, 7)\left\{k_{12}^{\mu} k_{12}^{\nu}+\sum_{j=3}^{7} k_{j}^{\mu} k_{j}^{\nu}\right\} .
\end{aligned}
$$

Additionally, we gather those terms which could not be lined up with multiparticle polarizations in the new $\Delta$ building block in the last line of (6.15),

$$
\begin{aligned}
\Delta_{8}(12 \mid 3,4,5,6,7)= & 2\left(k_{1} \cdot k_{2}\right)\left(\left(\epsilon_{1} \cdot \epsilon_{3}\right)\left(\epsilon_{2} \cdot\left(k_{3}-k_{1}\right)\right)-\left(\epsilon_{2} \cdot \epsilon_{3}\right)\left(\epsilon_{1} \cdot\left(k_{3}-k_{2}\right)\right)\right) t_{8}(4,5,6,7) \\
& +(3 \leftrightarrow 4,5,6,7) .
\end{aligned}
$$

This object is antisymmetric in the two labels to the left of the vertical bar, and as such contributes to the seven-point hexagon numerator where those two legs have been pulled out as the dangling tree. It would be interesting to relate (6.17) to a component version of the so-called refined building blocks $J$ in pure-spinor superspace $[63,110]$.

\section{3 $D=6$ examples}

Similar one-loop numerators can be given for $D=6$ SYM with half-maximal supersymmetry. We will focus on a hypermultiplet running in the loop, whose correlators have been expressed in terms of traces in (4.7). As a reminder, the particle content for the hypermultiplet is given by the parameter choices $\mathbf{n}_{\mathrm{v}}=0, \mathbf{n}_{\mathrm{f}}=1, \mathbf{n}_{\mathrm{s}}=2$, and the numerators will be defined via

$$
\mathcal{I}_{(0,1,2, D=6)}^{(1)}=\frac{1}{2} \sum_{\rho \in S_{n}} N_{\text {hyp }}^{(1)}(+, \rho(1,2, \ldots, n),-) \operatorname{PT}(+, \rho(1,2, \ldots, n),-) .
$$

As an analogue of the multiparticle $t_{8}$-tensor (6.8) that governs maximally supersymmetric numerators, the basic scalar building block for $D=6 \mathrm{SYM}$ is

$$
t_{4}(A, B)=-\frac{1}{2}\left(f_{A}\right)^{\mu \nu}\left(f_{B}\right)_{\mu \nu}=\frac{1}{2} \operatorname{tr}_{\mathrm{V}}(A, B) .
$$

\footnotetext{
${ }^{16}$ The coefficients $-\frac{1}{8}=\left(-\frac{1}{2}\right)^{3}$ and $-\frac{1}{12}=\frac{1}{6}\left(-\frac{1}{2}\right)$ of $t_{8}(12,34,56,7)$ and $t_{8}(123,45,6,7)$ can be anticipated by multiplying the combinatorial factors $-\frac{1}{2}$ and $\frac{1}{6}$ of the two- and three-particle slots in (6.12) and (6.13). The permutation sums include combinations $t_{8}(i j k, p q, \ldots)+t_{8}(k j i, p q, \ldots)$ with $1 \leq i<j<k<7$ and $1 \leq p<q \leq 7$ as well as $t_{8}(i j, p q, r s, \ldots)$ with $1 \leq i<j<7, \quad 1 \leq p<q<7, \quad 1 \leq r<s \leq 7$ and $i<p<r$ as well as $-t_{8}(i j k l, \ldots)+$ $t_{8}(l k j i, \ldots)+t_{8}(i l j k, \ldots)+t_{8}(j k i l, \ldots)$ with $1 \leq i<j<k<l<7$.
} 
Its simplest instance $t_{4}(1,2)=\left(k_{1} \cdot \epsilon_{2}\right)\left(k_{2} \cdot \epsilon_{1}\right)-\left(k_{1} \cdot k_{2}\right)\left(\epsilon_{1} \cdot \epsilon_{2}\right)$ vanishes in the momentum phase space of two massless particles, but we will find non-vanishing multiparticle examples. In particular, one can attain linearized gauge invariance at the level of loop integrands by relaxing momentum conservation: the numerators of this section are understood to rely on no Mandelstam identity other than $s_{12 \ldots n}=0$ at $n$ points. This proposal goes back to work of Minahan in 1987 [111] and will be referred to as Minahaning (also see [7, 58, 106] for four-point implementations).

At three points for instance, Minahaning amounts to keeping nonzero $s_{i j}$ while imposing $s_{12}+s_{13}+s_{23}=0$, and it introduces non-vanishing $s_{i j k}$ at four points. For dot products with polarization vectors, transversality and momentum conservation will be used as usual, i.e. $\left(\epsilon_{1} \cdot k_{12 \ldots n}\right)=\left(\epsilon_{1} \cdot k_{2 \ldots n}\right)=0$. These choices lead to $t_{4}(12,3)=\left(k_{1} \cdot k_{2}\right)\left(\epsilon_{1} \cdot \epsilon_{2}\right)\left(k_{1} \cdot \epsilon_{3}\right)$, where the factor of $\left(k_{1} \cdot k_{2}\right)$ cancels the formally divergent propagator $\left(k_{1}+k_{2}\right)^{-2}$ of a three-point diagram with an external bubble. More generally, any potentially divergent propagator introduced by Parke-Taylor integrals (i.e.forward limits of doubly-partial amplitudes) will be cancelled by the corresponding Mandelstam invariant from the numerators of this section. However, this mechanism does not cure forward-limit divergences in the tree-level propagators that arise when integrating non-supersymmetric correlators (4.4) in terms of doubly-partial amplitudes.

Similar to (6.9) and (6.10), the subsequent numerators are built from vector and tensor generalizations of the scalar building block (6.19),

$$
\begin{aligned}
t_{4}^{\mu}(A, B, C) & =\epsilon_{A}^{\mu} t_{4}(B, C)+\epsilon_{B}^{\mu} t_{4}(A, C)+\epsilon_{C}^{\mu} t_{4}(A, B) \\
t_{4}^{\mu \nu}(A, B, C, D) & =\left(\epsilon_{A}^{\mu} \epsilon_{B}^{\nu}+\epsilon_{A}^{\nu} \epsilon_{B}^{\mu}\right) t_{4}(C, D)+(A, B \mid A, B, C, D) \\
t_{4}^{\mu \nu \lambda}(A, B, C, D, E) & =\left(\epsilon_{A}^{\mu} \epsilon_{B}^{\nu} \epsilon_{C}^{\lambda}+\operatorname{symm}(\mu, \nu, \lambda)\right) t_{4}(D, E)+(A, B, C \mid A, B, C, D, E),
\end{aligned}
$$

which are again symmetric under exchange of multiparticle labels $t_{\ddot{4}}(A, B, \ldots)=$ $t_{4}(B, A, \ldots)$ and were firstly considered in the context of one-loop superstring amplitudes with reduced supersymmetry $[58,106]$. With these definitions, the BCJ numerators following from the correlator (4.7) include ${ }^{17}$

$$
\begin{aligned}
-N_{\text {hyp }}^{(1)}(+, 1,2,3,-)= & \ell_{\mu} t_{4}^{\mu}(1,2,3)-\frac{1}{2}\left\{t_{4}(12,3)+t_{4}(13,2)+t_{4}(23,1)\right\} \\
-N_{\text {hyp }}^{(1)}(+, 1,2,3,4,-)= & \frac{1}{2} \ell_{\mu} \ell_{\nu} t_{4}^{\mu \nu}(1,2,3,4)-\frac{1}{2} \ell_{\mu}\left\{t_{4}^{\mu}(12,3,4)+(1,2 \mid 1,2,3,4)\right\} \\
& +\frac{1}{6}\left\{t_{4}(123,4)+t_{4}(321,4)+(4 \leftrightarrow 1,2,3)\right\} \\
& +\frac{1}{4}\left\{t_{4}(12,34)+t_{4}(13,24)+t_{4}(14,23)\right\} \\
& +\widehat{t}_{8}(1,2,3,4)-\frac{1}{12} N_{\max }^{(1)}(+, 1,2,3,4,-)
\end{aligned}
$$

where the quantity $\widehat{t}_{8}(1,2,3,4)$ in the last line generalizes $(6.14)$ to half-maximal super-

\footnotetext{
${ }^{17}$ Similar to the earlier examples, the permutation sums in $(6.22)$ include all $t_{4}^{\mu}(i j, \ldots)$ with $1 \leq i<j \leq 4$ and $t_{4}(i j k, \ldots)+t_{4}(k j i, \ldots)$ with $1 \leq i<j<k \leq 4$.
} 
symmetry and does not coincide with the gauge-invariant $t_{8}(1,2,3,4)$ :

$$
\widehat{t}_{8}(1,2,3,4)=\frac{1}{12}\left\{\left(k_{2}^{\mu}-k_{1}^{\mu}\right) t_{4}^{\mu}(12,3,4)+(1,2 \mid 1,2,3,4)\right\}-\frac{1}{24} t_{4}^{\mu \nu}(1,2,3,4) \sum_{j=1}^{4} k_{j}^{\mu} k_{j}^{\nu} .
$$

By comparison with (6.12) and (6.13), the triangle and box numerator with half-maximal supersymmetry share the combinatorics of maximally supersymmetric pentagon and hexagon numerators. The examples (6.21) and (6.22) have been known from [44, 106], and the one-loop string-amplitude prescription implies ${ }^{18}$ that $n$-point amplitudes with half-maximal supersymmetry generally have the same complexity as $(n+2)$-point amplitudes with maximal supersymmetry [58]. Accordingly, the following pentagon numerator is inspired by the maximally supersymmetric heptagon numerator (6.15),

$$
\begin{aligned}
- & N_{\text {hyp }}^{(1)}(+, 1,2,3,4,5,-)= \\
& \frac{1}{6} \ell_{\mu} \ell_{\nu} \ell_{\lambda} t_{4}^{\mu \nu \lambda}(1,2,3,4,5)-\frac{1}{4} \ell_{\mu} \ell_{\nu}\left\{t_{4}^{\mu \nu}(12,3,4,5)+(1,2 \mid 1,2,3,4,5)\right\} \\
& +\frac{1}{4} \ell_{\mu}\left\{t_{4}^{\mu}(12,34,5)+t_{4}^{\mu}(13,24,5)+t_{4}^{\mu}(14,23,5)+(5 \leftrightarrow 1,2,3,4)\right\} \\
& +\frac{1}{6} \ell_{\mu}\left\{t_{4}^{\mu}(123,4,5)+t_{4}^{\mu}(321,4,5)+(4,5 \mid 1,2,3,4,5)\right\}+\ell_{\mu} \widehat{t}_{8}^{\mu}(1,2,3,4,5) \\
& -\frac{1}{2}\left\{\widehat{t}_{8}(12,3,4,5)+(1,2 \mid 1,2,3,4,5)\right\}-\frac{1}{12}\left\{t_{4}(123,45)+t_{4}(321,45)+(4,5 \mid 1,2,3,4,5)\right\} \\
& +\frac{1}{12}\left\{-t_{4}(1234,5)+t_{4}(4321,5)+t_{4}(1423,5)+t_{4}(2314,5)+(5 \leftrightarrow 1,2,3,4)\right\} \\
& -\frac{1}{96}\left\{\Delta_{4}(12 \mid 3,4,5)+(1,2 \mid 1,2,3,4,5)\right\}-\frac{1}{12} N_{\max }^{(1)}(+, 1,2,3,4,5,-),
\end{aligned}
$$

where by analogy with (6.16) and (6.17)

$$
\begin{aligned}
\widehat{t}_{8}^{\mu}(1,2,3,4,5)= & \frac{1}{12}\left\{\left(k_{2}^{\lambda}-k_{1}^{\lambda}\right) t_{4}^{\mu \lambda}(12,3,4,5)+(1,2 \mid 1,2,3,4,5)\right\} \\
& -\frac{1}{24} t_{4}^{\mu \nu \lambda}(1,2,3,4,5) \sum_{j=1}^{5} k_{j}^{\nu} k_{j}^{\lambda} \\
\widehat{t}_{8}(12,3,4,5)= & \frac{1}{12}\left\{\left(k_{3}^{\mu}-k_{12}^{\mu}\right) t_{4}^{\mu}(123,4,5)+\left(k_{4}^{\mu}-k_{3}^{\mu}\right) t_{4}^{\mu}(12,34,5)+\operatorname{cyc}(3,4,5)\right\} \\
& -\frac{1}{24} t_{4}^{\mu \nu}(12,3,4,5)\left\{k_{12}^{\mu} k_{12}^{\nu}+\sum_{j=3}^{5} k_{j}^{\mu} k_{j}^{\nu}\right\} \\
\Delta_{4}(12 \mid 3,4,5)= & 2\left(k_{1} \cdot k_{2}\right)\left(\left(\epsilon_{1} \cdot \epsilon_{3}\right)\left(\epsilon_{2} \cdot\left(k_{3}-k_{1}\right)\right)-\left(\epsilon_{2} \cdot \epsilon_{3}\right)\left(\epsilon_{1} \cdot\left(k_{3}-k_{2}\right)\right)\right) t_{4}(4,5)+(3 \leftrightarrow 4,5) .
\end{aligned}
$$

The appearance of the maximally supersymmetric pentagon numerator (6.12) in the last line of $(6.24)$ generalizes the $t_{8}(1,2,3,4)$ in the last line of $(6.22)$ to five points. The derivation of this new five-point result has been greatly facilitated by the representation (4.4) of the correlator induced by forward limits.

\footnotetext{
${ }^{18}$ More specifically, this property follows from the sum over spin structures in the RNS-prescription for one-loop amplitudes of conventional strings and ambitwistor strings. Depending on the amount of spacetime supersymmetry, the partition functions for given spin structures conspire to eliminate 2 or 4 singularities from the fermionic two-point functions when performing the spin sum [44, 58].
} 


\subsection{Parity-odd examples}

The forward-limit prescription (5.3) for parity-odd correlators can also be lined up with compact BCJ numerators in terms of multiparticle fields. On top of the simplest nonvanishing numerator (5.10) at multiplicity $n=\frac{D}{2}$, the correlator $(5.13)$ at $\frac{D}{2}+1$ points leads to the BCJ numerators

$$
\begin{aligned}
-i N_{\text {odd }}^{(1)}\left(+, 1,2, \ldots, \frac{D}{2}+1,-\right)= & \left\{\left(\ell \cdot \epsilon_{2}\right) \varepsilon_{D}\left(\ell, \epsilon_{1}, f_{3}, \ldots, f_{D / 2+1}\right)+\left(2 \leftrightarrow 3,4, \ldots, \frac{D}{2}+1\right)\right\} \\
& -\frac{1}{2}\left\{\varepsilon_{D}\left(\ell, \epsilon_{12}, f_{3}, \ldots, f_{D / 2+1}\right)+\left(2 \leftrightarrow 3,4, \ldots, \frac{D}{2}+1\right)\right\} \\
& -\frac{1}{2}\left\{\varepsilon_{D}\left(\ell, \epsilon_{1}, f_{23}, \ldots, f_{D / 2+1}\right)+\left(2,3 \mid 2,3, \ldots, \frac{D}{2}+1\right)\right\} .
\end{aligned}
$$

Similar expressions are expected at higher multiplicity, where (5.3) manifests that no more than $n+1-\frac{D}{2}$ powers of $\ell$ can occur in $n$-point numerators. In $D=4$, this leads to a power counting of $\ell^{n-3}$ in $N_{\text {odd }}^{(1)}$ that exceeds the $\ell^{n-2}$ in the parity-even part of numerators of chiral $\mathcal{N}=1$ SYM inferred from (4.9).

\section{$7 \quad$ Summary and outlook}

In this work, we have constructed streamlined representations of one-loop correlators in various gauge theories by taking forward limits of tree-level correlators. Our results are driven by new representations of two-fermion correlators at tree level which closely resemble their bosonic counterparts. The combination of their forward limits therefore manifests all supersymmetry cancellations, and the power counting of loop momenta follows from representation-theoretic identities between Lorentz traces over vector and spinor indices.

Our results apply to gauge-theory correlators in arbitrary even dimensions and with any combination of adjoint scalars, fermions and gauge bosons running in the loop. Also in the non-supersymmetric case, we expand the correlators in terms of Parke-Taylor factors in a subset of the external legs accompanied by Pfaffians. It is then straightforward to extract BCJ numerators w.r.t. linearized propagators by rearranging the Parke-Taylor factors according to well-established tree-level techniques in the $\mathrm{YM}+\phi^{3}$ theory.

A variety of interesting follow-up questions is left for the future, for instance:

- The strategy of this work calls for an application to higher-loop correlators, starting from the two-loop case on a bi-nodal Riemann sphere [39, 41]. It remains to identify suitable representations of tree-level correlators to perform multiple forward limits, and the four-fermion correlator in appendix B.4 could be a convenient starting point. The gluing operators of [65] and the discussion of double-forward limits in [42] will give crucial guidance in this endeavor.

- The Parke-Taylor decompositions of the one-loop correlators in this work lead to BCJ numerators w.r.t. linearized propagator in the loop momenta. Their algorithmic 
recombination to quadratic propagators is still an open problem (see [49-53] for recent progress in this direction) and has not yet been understood at the level of the $(n+2)$-point tree-level building blocks. We hope that our representations of BCJ numerators in general gauge theories provide helpful case studies to (i) pinpoint the key mechanisms in the conversion to quadratic propagators (ii) offer a way to preserve the BCJ duality in this process.

- The description of our one-loop BCJ numerators in terms of multiparticle fields has not yet been generalized to arbitrary multiplicity. Even though the Berends-Giele currents for tree-level subdiagrams in BCJ gauge are available to all multiplicity [60, 75], their composition rules in one-loop numerators involve additional structures. An all-multiplicity construction of one-loop BCJ numerators from multiparticle fields is likely to shed new light on the long-standing questions concerning a kinematic algebra.

\section{Acknowledgments}

We are grateful to Henrik Johansson, Carlos Mafra, Lionel Mason, Gustav Mogull, Ricardo Monteiro and Yong Zhang for combinations of inspiring discussions and collaboration on related topics. AE is supported by the Knut and Alice Wallenberg Foundation under KAW 2018.0116, From Scattering Amplitudes to Gravitational Waves. SH is supported in part by NSF of China under Grant No. 11947302 and 11935013. OS is supported by the European Research Council under ERC-STG-804286 UNISCAMP. FT is supported in part by the Knut and Alice Wallenberg Foundation under grant KAW 2013.0235, and the Ragnar Söderberg Foundation (Swedish Foundations' Starting Grant).

\section{A One-loop integrands with linear propagators and CHY formulas}

Throughout the paper, we adopt a non-standard representation for Feynman integrals of one-loop amplitudes, which naturally arises in one-loop CHY formulas or forward limits of tree amplitudes. Repeated partial-fraction manipulations of the standard Feynman propagators $(\ell+K)^{2}$ in one-loop integrals (with some linear combination $K$ of external momenta) allow to eliminate the reference to $\ell^{2}$ from all propagators except for one [36, 37]. It suffices to show the result for the massless $n$-gon $\left(s_{12 \ldots p, \pm \ell}=\sum_{1 \leq i<j}^{p} s_{i j} \pm \ell \cdot k_{12 \ldots p}\right.$ with $\left.k_{12 \ldots p}=\sum_{j=1}^{p} k_{j}\right)$,

$$
\begin{aligned}
& \int \frac{2^{n-1} \mathrm{~d}^{D} \ell}{\ell^{2}\left(\ell+k_{1}\right)^{2}\left(\ell+k_{12}\right)^{2} \ldots\left(\ell+k_{12 \ldots n-1}\right)^{2}}=\sum_{i=0}^{n-1} \int \frac{2^{n-1} \mathrm{~d}^{D} \ell}{\left(\ell+k_{12 \ldots i}\right)^{2}} \prod_{j \neq i} \frac{1}{\left(\ell+k_{12 \ldots j}\right)^{2}-\left(\ell+k_{12 \ldots i}\right)^{2}} \\
& =\sum_{i=0}^{n-1} \int \frac{\mathrm{d}^{D} \ell}{\ell^{2}} \prod_{j=0}^{i-1} \frac{1}{s_{j+1, j+2, \ldots, i,-\ell}} \prod_{j=i+1}^{n-1} \frac{1}{s_{i+1, i+2, \ldots, j, \ell}},
\end{aligned}
$$

where we have performed an $i$-dependent shift of the loop momentum $\ell \rightarrow \ell-k_{12 \ldots i}$ to uniformly obtain $\ell^{2}$ as the only quadratic propagator in the second line. As visualized in 


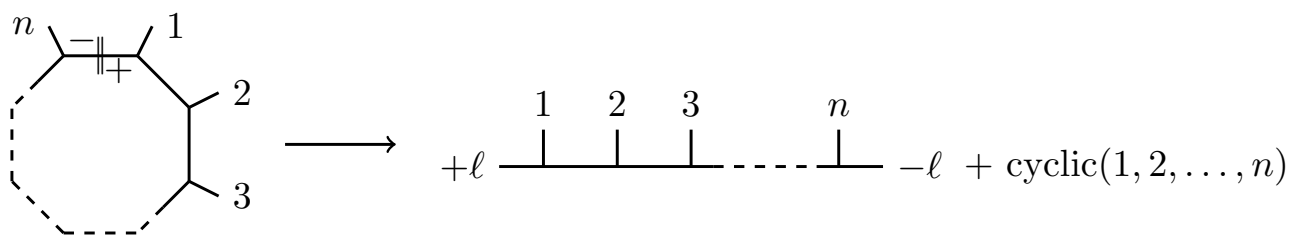

Figure 1. Interpretation of the partial-fraction representation of loop integrals as $(n+2)$-point tree-level diagrams.

figure 1 , each term in the sum over $i$ can be interpreted as one way of opening up the $n$-gon and is associated with an $(n+2)$-point tree diagram involving off-shell momenta $\pm \ell[38,45]$. Each of the cubic diagrams can have different kinematic numerators, leaving a total of $n$ ! inequivalent $n$-gon numerators.

The manipulations in (A.1) also apply to one-loop integrals with massive momenta $k_{A}, k_{B}, k_{C}$ and $k_{D}$ such as $k_{A}=k_{a_{1}}+k_{a_{2}}+\ldots+k_{a_{p}}$ for $A=a_{1} a_{2} \ldots a_{p}$. E.g. a massive box admits the following four-term representation:

$$
\int \frac{8 \mathrm{~d}^{D} \ell}{\ell^{2}\left(\ell+k_{A}\right)^{2}\left(\ell+k_{A B}\right)^{2}\left(\ell+k_{A B C}\right)^{2}}=\int \frac{\mathrm{d}^{D} \ell}{\ell^{2}}\left(\frac{1}{s_{A, \ell} s_{A B, \ell} s_{D,-\ell}}+\operatorname{cyc}(A, B, C, D)\right)
$$

These rearrangements uniquely decompose the one-loop integrand for color-ordered singletrace amplitudes into $n$ terms dubbed partial integrands [43], similar to the decomposition (A.1) of the $n$-gon. Each partial integrand can be interpreted as the forward limit of a color-ordered $(n+2)$-point tree amplitude with off-shell momenta, where for instance the momenta of the two legs between $n$ and 1 in figure 1 are identified as $\ell$ and $-\ell$ [38]. Although it is an open problem to perform loop integrals over linearized propagators, the above rearrangements of loop integrals have to yield the same result as integrating the quadratic propagators.

Such integrals naturally arise from one-loop CHY formulas, which can be obtained by performing forward limits on tree-level CHY formulas, or by localizing the $\tau$ integral of ambitwistor-string formulas at genus one [30] at the cusp $\tau \rightarrow i \infty$, where the torus degenerates to a nodal sphere $[36,37]$. A general formula for e.g. one-loop amplitudes of gravity and gauge theories in $D$ spacetime dimensions reads (with the normalization factor $\mathscr{N}$ from the $(n+2)$-point tree amplitude (1.1))

$$
\mathcal{M}_{L \otimes R}^{(1)}=\mathscr{N} \int \frac{\mathrm{d}^{D} \ell}{\ell^{2}} \int \frac{\mathrm{d} \sigma_{+} \mathrm{d} \sigma_{-} \mathrm{d}^{n} \sigma_{i}}{\operatorname{Vol}[\operatorname{SL}(2, \mathbb{C})]} \prod_{i=+,-1}^{n} \delta\left(E_{i}\right) \mathcal{I}_{L}^{(1)}(\ell) \mathcal{I}_{R}^{(1)}(\ell)
$$

where $E_{i}$ is the $i$-th tree-level scattering equation of $(n+2)$ points and we take forward limit by $k_{ \pm} \rightarrow \pm \ell$. As indicated by the prime, three of the equations are redundant due to the $\operatorname{SL}(2, \mathbb{C})$ symmetry. For gauge theories, one of the two half integrands $\mathcal{I}_{L}^{(1)}(\ell) \rightarrow \mathcal{I}_{U(N)}^{(1)}$

$$
\mathcal{I}_{U(N)}^{(1)}=\sum_{\rho \in S_{n-1}} \operatorname{Tr}\left(t^{a_{1}} t^{a_{\rho(2)}} t^{a_{\rho(3)}} \ldots t^{a_{\rho(n)}}\right) \mathrm{PT}^{(1)}(1, \rho(2,3, \ldots, n))
$$


is a sum of color traces accompanied by one-loop analogues $\mathrm{PT}^{(1)}$ of Parke-Taylor factors,

$$
\mathrm{PT}^{(1)}(1,2, \ldots, n) \equiv \frac{1}{\sigma_{-, 1} \sigma_{1,2} \sigma_{2,3} \ldots \sigma_{n-1, n} \sigma_{n,+} \sigma_{+,-}}+\operatorname{cyc}(1,2, \ldots, n) .
$$

Throughout the paper we are interested in the other, polarization-dependent half-integrand $\mathcal{I}_{R}^{(1)} \rightarrow \mathcal{I}_{\mathrm{SYM}}^{(1)}(\ell)$ with our main results in (3.15) and (4.4). By expanding it as a linear combination of $(n+2)$-point Parke-Taylor factors, the coefficients become BCJ master numerators for one of the $n$ terms in the decomposition of one-loop $n$-gon in (A.1). In terms of the two half-integrands, one-loop amplitudes (A.3) in gauge theory and (super-)gravity are obtained as $\mathcal{M}_{U(N) \otimes \mathrm{SYM}}^{(1)}$ and $\mathcal{M}_{\mathrm{SYM} \otimes \mathrm{SYM}}^{(1)}$, respectively.

Note that these integrals with linearized propagators not only naturally appear from CHY formulas, but also enter the Q-cut representation of loop amplitudes [112]. Such representations provide a well-defined notion of "loop integrands" for non-planar diagrams and generic theories ${ }^{19}$ and offer valuable perspectives on the structure of loop amplitudes. It also allows one to generalize KLT and BCJ relations to one loop [43, 44].

\section{B Conformal field theory and tree-level correlators}

\section{B.1 CFT basics}

In the worldsheet conformal field theory (CFT) of the RNS formalism in $D=10$, the free-field OPEs relevant for the correlators of gluon vertex operators (2.1) read

$$
\begin{aligned}
\psi^{\mu}(\sigma) \psi^{\nu}(0) & \sim \frac{\eta^{\mu \nu}}{\sigma}, & e^{p \phi}(\sigma) e^{q \phi}(0) & \sim \sigma^{-p q} e^{(p+q) \phi} \\
P^{\mu}(\sigma) X^{\nu}(0) & \sim-i \frac{\eta^{\mu \nu}}{\sigma}, & P^{\mu}(\sigma) e^{i k \cdot X}(0) & \sim \frac{k^{\mu} e^{i k \cdot X}}{\sigma} .
\end{aligned}
$$

The spin field in the fermion vertex (2.2) interacts with worldsheet spinor $\psi^{\mu}$ via

$$
S_{\alpha}(\sigma) S^{\beta}(0) \sim \frac{\delta_{\alpha}^{\beta}}{\sigma^{5 / 4}}, \quad S_{\alpha}(\sigma) S_{\beta}(0) \sim \frac{\gamma_{\alpha \beta}^{\mu} \psi_{\mu}(0)}{\sqrt{2} \sigma^{3 / 4}} .
$$

As a result of the OPEs, we have two- and three-point correlation functions $\left(\sigma_{i j}=\sigma_{i}-\sigma_{j}\right)$

$$
\begin{aligned}
\left\langle S_{\alpha} e^{-\phi / 2}\left(\sigma_{1}\right) S^{\beta} e^{-3 \phi / 2}\left(\sigma_{2}\right)\right\rangle & =\frac{\delta_{\alpha}^{\beta}}{\sigma_{12}^{2}} \\
\left\langle S_{\alpha} e^{-\phi / 2}\left(\sigma_{1}\right) S_{\beta} e^{-\phi / 2}\left(\sigma_{2}\right) \psi^{\mu} e^{-\phi}\left(\sigma_{3}\right)\right\rangle & =\frac{\gamma_{\alpha \beta}^{\mu}}{\sqrt{2} \sigma_{12} \sigma_{13} \sigma_{23}},
\end{aligned}
$$

see [113] for higher-point spin-field correlators in various dimensions.

The conformal fields $\psi^{\mu}$ and $S_{\alpha}$ are primary fields of a Kac-Moody current algebra at level $k=1$ with generators $\psi^{\mu} \psi^{\nu}$. By Kac-Moody Ward identities, current insertions in a correlator can be removed by summing over all OPE singularities such as $[81,83,85]$

$$
\begin{aligned}
\psi^{\mu} \psi^{\nu}(\sigma) \psi^{\lambda}(0) & \sim \frac{2 \psi^{[\mu} \eta^{\nu] \lambda}(0)}{\sigma}, \quad \psi^{\mu} \psi^{\nu}(\sigma) S_{\alpha}(0) \sim-\frac{\gamma^{\mu \nu}{ }_{\alpha}{ }^{\beta} S_{\beta}(0)}{2 \sigma} \\
\psi^{\mu} \psi^{\nu}(\sigma) \psi^{\lambda} \psi^{\rho}(0) & \sim \frac{2 \eta^{\lambda[\nu} \eta^{\mu] \rho}}{\sigma^{2}}+\frac{2\left(\eta^{\lambda[\nu} \psi^{\mu]} \psi^{\rho}-\eta^{\rho \nu} \psi^{\mu]} \psi^{\lambda}\right)(0)}{\sigma},
\end{aligned}
$$

\footnotetext{
${ }^{19}$ Also see [23] for the emergence of global loop integrands from the field-theory limit of string amplitudes.
} 
with the normalization conventions $2 \eta^{\lambda[\nu} \eta^{\mu] \rho}=\eta^{\lambda \nu} \eta^{\mu \rho}-\eta^{\lambda \mu} \eta^{\nu \rho}$ for antisymmetrization brackets. Hence, current-algebra techniques can be used to straightforwardly compute spin-field correlators with any number of $\psi_{\mu} \psi_{\nu}$ insertions. In this way, the contributions $\sim f^{\mu \nu} \psi_{\mu} \psi_{\nu}(\sigma)$ of bosonic vertex operator (2.1) in the zero picture can be addressed in presence of spin fields.

\section{B.2 Bosonic correlators and the Pfaffian}

When the bosonic correlator (2.4) is evaluated as the reduced Pfaffian in (2.5), the antisymmetric $2 n \times 2 n$ matrix $\Psi_{\{12 \ldots n\}}$ is organized into $n \times n$ blocks A, B and C [26]

$$
\Psi=\left(\begin{array}{cc}
\mathrm{A} & -\mathrm{C}^{T} \\
\mathrm{C} & \mathrm{B}
\end{array}\right)_{2 n \times 2 n}
$$

with $\mathrm{C}^{T}$ denoting the transpose of $\mathrm{C}$. The entries of the $n \times n$ matrices A, B, C are given by ${ }^{20}$

$$
\mathrm{A}_{i j}=\left\{\begin{array}{cc}
\frac{k_{i} \cdot k_{j}}{\sigma_{i j}} & i \neq j, \\
0 & i=j,
\end{array} \quad \mathrm{~B}_{i j}=\left\{\begin{array}{cc}
\frac{\epsilon_{i} \cdot \epsilon_{j}}{\sigma_{i j}} & i \neq j, \\
0 & i=j,
\end{array} \quad \mathrm{C}_{i j}=\left\{\begin{array}{cc}
-\frac{\epsilon_{i} \cdot k_{j}}{\sigma_{i j}} & i \neq j, \\
\sum_{\substack{p=1 \\
p \neq i}}^{n} \frac{\epsilon_{i} \cdot k_{p}}{\sigma_{i p}} & i=j .
\end{array}\right.\right.\right.
$$

We define the Pfaffian of a $2 n \times 2 n$ anti-symmetric matrix as

$$
\operatorname{Pf} \Psi=(-1)^{\frac{n(n+1)}{2}} \frac{1}{2^{n} n !} \sum_{\rho \in S_{2 n}} \operatorname{sign}(\rho) \prod_{i=1}^{n} \Psi_{\rho(2 i-1) \rho(2 i)} .
$$

As a consequence of momentum conservation and scattering equations, the matrix $\Psi$ has two null vectors such that $\operatorname{Pf} \Psi=0$. The reduced Pfaffian in (2.5), by contrast, yields a non-vanishing bosonic correlator on the support of momentum conservation and scattering equations.

The diagonal terms of the C-matrix in (B.9) arise when the first term $\sim \epsilon^{\mu} P_{\mu}(\sigma)$ in $V^{(0)}$ contracts the plane waves of the remaining vertex operators,

$$
\left\langle\epsilon_{j}^{\mu} P_{\mu}\left(\sigma_{j}\right) \prod_{i=1}^{n} e^{i k_{i} \cdot X\left(\sigma_{i}\right)}\right\rangle=\mathrm{C}_{j j}\left\langle\prod_{i=1}^{n} e^{i k_{i} \cdot X\left(\sigma_{i}\right)}\right\rangle, \quad \mathrm{C}_{j j}=\sum_{\substack{i=1 \\ i \neq j}}^{n} \frac{\epsilon_{j} \cdot k_{i}}{\sigma_{j i}},
$$

see (B.2) for the underlying OPEs. Accordingly, when multiple $V^{(0)}$ contribute through the conformal field $P_{\mu}$, the plane-wave correlators relevant to any number of bosons and fermions evaluate to

$$
\left\langle\left(\prod_{j=1}^{m} \epsilon_{j}^{\mu} P_{\mu}\left(\sigma_{j}\right)\right) \prod_{i=1}^{n} e^{i k_{i} \cdot X\left(\sigma_{i}\right)}\right\rangle=\left(\prod_{j=1}^{m} \mathrm{C}_{j j}\right)\left\langle\prod_{i=1}^{n} e^{i k_{i} \cdot X\left(\sigma_{i}\right)}\right\rangle .
$$

\footnotetext{
${ }^{20}$ The $\mathrm{C}$ matrix defined here differs from that in the original CHY paper [25] and appendix A of [72]. In particular, $C_{\text {here }}=-C_{\text {there }}$ such that the expansions $(2.6),(2.11)$ and (2.16) do not involve alternating signs, cf. the corresponding equations in [72].
} 
This is the CFT origin of those term in the correlators (2.6), (2.11) and (2.16), where the Pfaffian $\operatorname{Pf} \Psi_{A}$ contributes via products of the $C_{j j}$ for all the labels in the set $A$ [28]. The admixtures of the A- and B-blocks in (B.9) as well as the non-diagonal $\mathrm{C}_{i j}$ at $i \neq j$ will be discussed in the next subsections.

\section{B.3 Two-fermion correlators}

For the first representation (2.11) of the fermionic vertex operator, the three-point example spelt out in (2.14) is an immediate consequence of the spin-field correlator (B.5). We shall now derive the contributions from the additional insertions of $V_{j}^{(0)}\left(\sigma_{j}\right)$ at $n \geq 4$ points from the recursive techniques outlined above.

At four points, the first term $V_{2}^{(0)}\left(\sigma_{2}\right) \rightarrow \epsilon_{2} \cdot P\left(\sigma_{2}\right)$ can be straightforwardly addressed via (B.11) and yields $C_{22}=\Psi_{\{2\}}$. Together with the accompanying spin-field correlator (B.5), we obtain $\Psi_{\{2\}} \mathcal{I}_{2 \mathrm{f}}^{\text {tree }}\left(1_{f}, 3_{f}, \hat{4}\right)$ and reproduce the first term in (2.15). The second term $V_{2}^{(0)}\left(\sigma_{2}\right) \rightarrow \frac{1}{2} f_{2}^{\mu \nu} \psi_{\mu} \psi_{\nu}\left(\sigma_{2}\right)$ requires the summation of the OPEs (B.6) according to the Kac-Moody Ward identity

$$
\begin{aligned}
\left\langle S_{\alpha}\left(z_{1}\right) \psi^{\mu} \psi^{\nu}\left(z_{2}\right) S_{\beta}\left(z_{3}\right) \psi^{\lambda}\left(z_{4}\right)\right\rangle=-\frac{\gamma^{\mu \nu} \delta}{2 \sigma_{21}}\left\langle S_{\delta}\left(z_{1}\right) S_{\beta}\left(z_{3}\right) \psi^{\lambda}\left(z_{4}\right)\right\rangle & \\
& -\frac{\gamma^{\mu \nu}{ }^{\delta}}{2 \sigma_{23}}\left\langle S_{\alpha}\left(z_{1}\right) S_{\delta}\left(z_{3}\right) \psi^{\lambda}\left(z_{4}\right)\right\rangle+\frac{2}{\sigma_{24}}\left\langle S_{\alpha}\left(z_{1}\right) S_{\beta}\left(z_{3}\right) \psi^{[\mu}\left(z_{4}\right)\right\rangle \eta^{\nu] \lambda} \\
= & \frac{1}{2 \sqrt{2} \sigma_{13}^{3 / 4} \sigma_{14}^{1 / 2} \sigma_{34}^{1 / 2}}\left\{\left(\frac{1}{\sigma_{24}}-\frac{1}{\sigma_{21}}\right)\left(\gamma^{\mu \nu} \gamma^{\lambda}\right)_{\alpha \beta}+\left(\frac{1}{\sigma_{24}}-\frac{1}{\sigma_{23}}\right)\left(\gamma^{\mu \nu} \gamma^{\lambda}\right)_{\beta \alpha}\right\} .
\end{aligned}
$$

In passing to the last line, we have inserted the three-point correlator (B.5) and used the gamma-matrix identity $\gamma_{\alpha \beta}^{\mu} \eta^{\nu \lambda}-\gamma_{\alpha \beta}^{\nu} \eta^{\mu \lambda}=\frac{1}{2}\left(\gamma^{\mu \nu} \gamma^{\lambda}\right)_{\alpha \beta}+\frac{1}{2}\left(\gamma^{\mu \nu} \gamma^{\lambda}\right)_{\beta \alpha}$. Upon contraction with $\frac{1}{2} \chi_{1}^{\alpha} f_{2}^{\mu \nu} \chi_{3}^{\beta} \epsilon_{4}^{\lambda}$ and dressing with the superghost correlator, this reproduces the last two terms in (2.15) and completes the derivation of the four-point correlator

$$
\mathcal{I}_{2 \mathrm{f}}^{\text {tree }}\left(1_{f}, 2,3_{f}, \hat{4}\right)=\operatorname{Pf} \Psi_{\{2\}} \frac{\left(\chi_{1} \phi_{4} \chi_{3}\right)}{\sigma_{14} \sigma_{43} \sigma_{31}}+\frac{\left(\chi_{1} f_{2} \notin_{4} \chi_{3}\right)}{\sigma_{12} \sigma_{24} \sigma_{43} \sigma_{31}}+\frac{\left(\chi_{1} \notin_{4} f_{2} \chi_{3}\right)}{\sigma_{14} \sigma_{42} \sigma_{23} \sigma_{31}} .
$$

Starting from five points, we encounter double-insertions of the current $\psi_{\mu} \psi_{\nu}$, and KacMoody Ward identities involve the double-pole in their OPE (B.7). These double-poles are attributed to the Pfaffians in (2.6), (2.11) \& (2.16) and yield the entries of the A- and B-blocks in $\operatorname{Pf} \Psi_{\{12 \ldots m\}}=\prod_{j=1}^{m} C_{j j}+\mathcal{O}\left(\mathrm{A}_{i j}, \mathrm{~B}_{i j}\right)$. More specifically, the expression (2.11) for the five-point correlator involves the two-particle Pfaffian

$$
\operatorname{Pf} \Psi_{\{2,3\}}=\mathrm{C}_{22} \mathrm{C}_{33}-\mathrm{C}_{23} \mathrm{C}_{32}-\mathrm{A}_{23} \mathrm{~B}_{23}=\mathrm{C}_{22} \mathrm{C}_{33}+\frac{\left(\epsilon_{2} \cdot k_{3}\right)\left(\epsilon_{3} \cdot k_{2}\right)-\left(k_{2} \cdot k_{3}\right)\left(\epsilon_{2} \cdot \epsilon_{3}\right)}{\sigma_{23}^{2}}
$$

where the last two terms arise from the double-pole terms in the Ward identity

$$
\left\langle S_{\alpha}\left(z_{1}\right) \psi^{\mu} \psi^{\nu}\left(z_{2}\right) \psi^{\lambda} \psi^{\rho}\left(z_{3}\right) S_{\beta}\left(z_{4}\right) \psi^{\tau}\left(z_{5}\right)\right\rangle_{\mathrm{poles}}^{\text {double }}=\frac{2 \eta^{\lambda[\nu} \eta^{\mu] \rho}}{\sigma_{23}^{2}}\left\langle S_{\alpha}\left(z_{1}\right) S_{\beta}\left(z_{4}\right) \psi^{\tau}\left(z_{5}\right)\right\rangle \text {. }
$$


The simple-pole terms in turn are given by

$$
\begin{aligned}
& \left\langle S_{\alpha}\left(z_{1}\right) \psi^{\mu} \psi^{\nu}\left(z_{2}\right) \psi^{\lambda} \psi^{\rho}\left(z_{3}\right) S_{\beta}\left(z_{4}\right) \psi^{\tau}\left(z_{5}\right)\right\rangle_{\text {poles }}^{\text {simple }}=-\frac{\gamma^{\mu \nu} \alpha^{\delta}}{2 \sigma_{21}}\left\langle S_{\delta}\left(z_{1}\right) \psi^{\lambda} \psi^{\rho}\left(z_{3}\right) S_{\beta}\left(z_{4}\right) \psi^{\tau}\left(z_{5}\right)\right\rangle \\
& -\frac{\gamma^{\mu \nu}{ }_{\beta} \delta}{2 \sigma_{24}}\left\langle S_{\alpha}\left(z_{1}\right) \psi^{\lambda} \psi^{\rho}\left(z_{3}\right) S_{\delta}\left(z_{4}\right) \psi^{\tau}\left(z_{5}\right)\right\rangle+\frac{2}{\sigma_{23}}\left(\eta^{\lambda[\nu}\left\langle S_{\alpha}\left(z_{1}\right) \psi^{\mu]} \psi^{\rho}\left(z_{3}\right) S_{\beta}\left(z_{4}\right) \psi^{\tau}\left(z_{5}\right)\right\rangle-(\lambda \leftrightarrow \rho)\right) \\
& +\frac{2}{\sigma_{25}}\left\langle S_{\alpha}\left(z_{1}\right) \psi^{\lambda} \psi^{\rho}\left(z_{3}\right) S_{\beta}\left(z_{4}\right) \psi^{[\mu}\left(z_{5}\right)\right\rangle \eta^{\nu] \tau}
\end{aligned}
$$

and recurse to the simpler correlators we have already evaluated in (B.13). Once the permutations of $\eta^{\lambda \nu} \gamma^{\mu \rho}$ are rewritten as a commutator $\left[\gamma^{\mu \nu}, \gamma^{\lambda \rho}\right]$, we arrive at

$$
\begin{aligned}
& \left\langle S_{\alpha}\left(z_{1}\right) \psi^{\mu} \psi^{\nu}\left(z_{2}\right) \psi^{\lambda} \psi^{\rho}\left(z_{3}\right) S_{\beta}\left(z_{4}\right) \psi^{\tau}\left(z_{5}\right)\right\rangle_{\text {poles }}^{\text {simple }}=\frac{1}{4 \sqrt{2} z_{14}^{3 / 4} z_{15}^{1 / 2} z_{45}^{1 / 2}} \\
& \quad \times\left\{\frac{z_{15}}{z_{12} z_{23} z_{35}}\left(\gamma^{\mu \nu} \gamma^{\lambda \rho} \gamma^{\tau}\right)_{\alpha \beta}+\frac{z_{15} z_{45}}{z_{12} z_{25} z_{34} z_{35}}\left(\gamma^{\mu \nu} \gamma^{\tau} \gamma^{\lambda \rho}\right)_{\alpha \beta}+\frac{z_{45}}{z_{42} z_{23} z_{35}}\left(\gamma^{\tau} \gamma^{\lambda \rho} \gamma^{\mu \nu}\right)_{\alpha \beta}\right. \\
& \left.\quad+\frac{z_{15}}{z_{13} z_{32} z_{25}}\left(\gamma^{\lambda \rho} \gamma^{\mu \nu} \gamma^{\tau}\right)_{\alpha \beta}+\frac{z_{15} z_{45}}{z_{13} z_{35} z_{24} z_{25}}\left(\gamma^{\lambda \rho} \gamma^{\tau} \gamma^{\mu \nu}\right)_{\alpha \beta}+\frac{z_{45}}{z_{43} z_{32} z_{25}}\left(\gamma^{\tau} \gamma^{\mu \nu} \gamma^{\lambda \rho}\right)_{\alpha \beta}\right\}
\end{aligned}
$$

after partial-fraction manipulations of the form $\left(\sigma_{12} \sigma_{23}\right)^{-1}+\operatorname{cyc}(1,2,3)=0$. Upon contraction with $\frac{1}{4} \chi_{1}^{\alpha} f_{2}^{\mu \nu} f_{3}^{\lambda \rho} \chi_{4}^{\beta} \epsilon_{5}^{\tau}$ and dressing with the superghost correlator, this reproduces the $A=\emptyset$ contribution to (2.11). Terms with $A=\{2\}$ and $A=\{3\}$ are easily checked by combining (B.11) with (B.13), and the Pfaffian associated with $A=\{2,3\}$ has been determined in (B.15) and (B.16). This completes the derivation of the five-point correlator.

The detailed five-point calculation exemplifies the CFT origin of the gamma-matrix products in the $n$-point correlator (2.11): they arise from the simple poles in the OPEs (B.6) and (B.7) that govern the recursive evaluation of spin-field correlators with an arbitrary number of Lorentz-current insertions. The latter capture the contributions $V_{j}^{(0)}\left(\sigma_{j}\right) \rightarrow$ $\frac{1}{2} f_{j}^{\mu \nu} \psi_{\mu} \psi_{\nu}\left(\sigma_{j}\right)$ which are converted to $\frac{1}{4} f_{j}^{\mu \nu}\left(\gamma_{\mu \nu}\right)_{\alpha}{ }^{\beta} S_{\beta}\left(\sigma_{1}\right) / \sigma_{1 j}$ when performing the OPE (B.6) with a spin field. The double-poles in the OPE (B.7) among Lorentz currents in turn promote the contributions $\prod_{j \in A} \mathrm{C}_{j j}$ from $V_{j}^{(0)}\left(\sigma_{j}\right) \rightarrow \epsilon_{j}^{\mu} P_{\mu}\left(\sigma_{j}\right)$ to $\operatorname{Pf} \Psi_{A}$ along the lines of (B.15).

Iterating these OPEs leads to products of gamma matrices, where the multiplication order is correlated with the labels of the accompanying $\sigma_{i j}^{-1}$. Partial-fraction manipulations and the commutators of $\gamma^{\mu \nu}$ can be used to arrive at the same number of gamma matrices and at a chain-structure $\left(\ldots \sigma_{i j} \sigma_{j k} \sigma_{k l} \ldots\right)^{-1}$ in each term. By analyzing the combinatorics of this algorithm and keeping in mind that the correlator does not depend on the order in which the $\psi^{\mu} \psi^{\nu}$ are eliminated via Ward identities, one arrives at the $n$-point expression in (2.11). The same logic has been used in deriving the $n$-point tree-level correlator in the pure-spinor formalism [14], where the double-pole contributions have been absorbed to redefine the kinematic factors of the simple poles and to eventually obtain multiparticle superfields.

The same way of applying Kac-Moody Ward identities gives rise to the alternative form (2.16) of the two-fermion correlator. For instance, the three-point correlator in (2.17) follows from the same use of Ward identities that eliminated a single Lorentz current 
in (B.13). On the one hand, the three-point correlator involving fermionic ghost pictures $V^{(-1 / 2)} V^{(-3 / 2)}$ shares certain intermediate steps with the four-point correlator from $V^{(-1 / 2)} V^{(-1 / 2)}$. On the other hand, we can give the same kind of all-multiplicity results (2.11) and (2.16) for both ghost-picture assignments. The discussion in section 3.1 illustrates that (2.16) due to $n-2$ insertions of $V^{(0)}$ instead of $n-3$ is more suitable to manifest the interplay with the bosonic correlator (2.6) upon forward limits.

These techniques to successively remove insertions of $\psi^{\mu} \psi^{\nu}$ from the correlator are universal to the $S O(D)$ Kac-Moody symmetry of the RNS model $[81,83,85]$ in any number of spacetime dimensions $D$. Since the Clifford algebra (2.13) also takes the same form in any number of dimensions, the structure of the gamma-matrix product in the two-fermion correlators (2.11) and (2.16) is universal to any even value of $D$. The only $D$-dependent aspect of these correlators is the relative chirality of the fermion wavefunctions $\chi_{j}$ which can be understood from the three-point correlator (B.5) for lower-dimensional spin fields that initiates the recursion based on Ward identities. The $D$-dimensional three-point correlator is nonzero in case of alike chiralities in $D=2 \bmod 4$ and opposite chiralities in $D=4 \bmod 4$, see e.g.section 3 of [113]. Hence, the two-fermion correlators in (2.11) and (2.16) can be used in any even $D \leq 10$ provided that one of the chiralities is flipped in $D=4 \bmod 4$.

\section{B.4 Four-fermion correlators}

The recursive computation of two-fermion correlators can be straightforwardly extended to the four-fermion case. In this case, Ward identities reduce correlators with Lorentz-current insertions to the basic spin-field correlator

$$
\left\langle S_{\alpha} e^{-\frac{\phi}{2}}\left(\sigma_{1}\right) S_{\beta} e^{-\frac{\phi}{2}}\left(\sigma_{2}\right) S_{\gamma} e^{-\frac{\phi}{2}}\left(\sigma_{3}\right) S_{\delta} e^{-\frac{\phi}{2}}\left(\sigma_{4}\right)\right\rangle=\frac{1}{2 \sigma_{41} \sigma_{23}}\left(\frac{\gamma_{\mu \alpha \beta} \gamma_{\gamma \delta}^{\mu}}{\sigma_{12} \sigma_{34}}+\frac{\gamma_{\mu \alpha \gamma} \gamma_{\beta \delta}^{\mu}}{\sigma_{13} \sigma_{24}}\right)
$$

Note that this result is specific to $D=10$ dimensions, see $[85,113]$ for the tensor structure of lower dimensional four-spin-field correlators. Permutation invariance under exchange of $\left(\alpha, \sigma_{1}\right) \leftrightarrow\left(\beta, \sigma_{2}\right)$ is obscured on the right-hand side of (B.19) but can be checked using the gamma matrix identity $\gamma_{\mu(\alpha \beta} \gamma_{\gamma) \delta}^{\mu}=0$ in ten dimensions. It can be manifested by rewriting the correlator as a reduced determinant with entries $\gamma_{\alpha \beta}^{\mu} / \sigma_{12}$.

As an immediate consequence of (B.19), the four-fermion correlator is given by

$$
\left\langle V_{1}^{\left(-\frac{1}{2}\right)}\left(\sigma_{1}\right) V_{2}^{\left(-\frac{1}{2}\right)}\left(\sigma_{2}\right) V_{3}^{\left(-\frac{1}{2}\right)}\left(\sigma_{3}\right) V_{4}^{\left(-\frac{1}{2}\right)}\left(\sigma_{4}\right)\right\rangle=\frac{\left(\chi_{1} \gamma^{\mu} \chi_{2}\right)\left(\chi_{3} \gamma_{\mu} \chi_{4}\right)}{4 \sigma_{12} \sigma_{23} \sigma_{34} \sigma_{41}}+\frac{\left(\chi_{1} \gamma^{\mu} \chi_{3}\right)\left(\chi_{2} \gamma_{\mu} \chi_{4}\right)}{4 \sigma_{13} \sigma_{32} \sigma_{24} \sigma_{41}} .
$$

Additional bosonic vertex operators yield the same contributions of $C_{j j}$ from $V_{j}^{(0)}\left(\sigma_{j}\right) \rightarrow$ $\epsilon_{j}^{\mu} P_{\mu}\left(\sigma_{j}\right)$ and the same $f_{j}$ contractions from $V_{j}^{(0)}\left(\sigma_{j}\right) \rightarrow \frac{1}{2} f_{j}^{\mu \nu} \psi_{\mu} \psi_{\nu}\left(\sigma_{j}\right)$ as detailed in the two-fermion case. For instance, the five-point correlator is obtained in the following form

$$
\begin{gathered}
\left\langle V_{1}^{\left(-\frac{1}{2}\right)}\left(\sigma_{1}\right) V_{2}^{\left(-\frac{1}{2}\right)}\left(\sigma_{2}\right) V_{3}^{\left(-\frac{1}{2}\right)}\left(\sigma_{3}\right) V_{4}^{\left(-\frac{1}{2}\right)}\left(\sigma_{4}\right) V_{5}^{(0)}\left(\sigma_{5}\right)\right\rangle \\
=\frac{1}{4 \sigma_{12} \sigma_{23} \sigma_{34} \sigma_{41}}[ \\
{\left[\operatorname{Pf} \Psi_{\{5\}}\left(\chi_{1} \gamma^{\mu} \chi_{2}\right)\left(\chi_{3} \gamma_{\mu} \chi_{4}\right)+\frac{\left(\chi_{1} f_{5} \gamma^{\mu} \chi_{2}\right)\left(\chi_{3} \gamma_{\mu} \chi_{4}\right)}{\sigma_{15}}+\frac{\left(\chi_{1} \gamma^{\mu} f_{5} \chi_{2}\right)\left(\chi_{3} \gamma_{\mu} \chi_{4}\right)}{\sigma_{52}}\right.} \\
\left.+\frac{\left(\chi_{1} \gamma^{\mu} \chi_{2}\right)\left(\chi_{3} f_{5} \gamma_{\mu} \chi_{4}\right)}{\sigma_{35}}+\frac{\left(\chi_{1} \gamma^{\mu} \chi_{2}\right)\left(\chi_{3} \gamma_{\mu} f_{5} \chi_{4}\right)}{\sigma_{54}}\right]+(2 \leftrightarrow 3) .
\end{gathered}
$$


Note that the exchange of 2 and 3 acts on both the $\chi_{j}$ and on the punctures in the fourpoint Parke-Taylor factor as well as the $\sigma_{i j}^{-1}$ inside the square brackets. One may eliminate one of the field-strength contractions via

$$
\left(\chi_{1} f_{5} \gamma^{\mu} \chi_{2}\right)\left(\chi_{3} \gamma_{\mu} \chi_{4}\right)-\left(\chi_{1} \gamma^{\mu} f_{5} \chi_{2}\right)\left(\chi_{3} \gamma_{\mu} \chi_{4}\right)+\left(\chi_{1} \gamma^{\mu} \chi_{2}\right)\left(\chi_{3} f_{5} \gamma_{\mu} \chi_{4}\right)-\left(\chi_{1} \gamma^{\mu} \chi_{2}\right)\left(\chi_{3} \gamma_{\mu} f_{5} \chi_{4}\right)=0
$$

to manifest the quadratic falloff as $\sigma_{5} \rightarrow \infty$, but we chose to display (B.21) in the more symmetric form, where the generalization to higher multiplicity is more apparent. Similar to the two-fermion case, the general formula is then given by a sum over all subsets $A$ of the bosons $\{5,6, \ldots, n\}$ along with $\operatorname{Pf} \Psi_{A}$. For a fixed choice of $A$, it remains to sum over all possibilities to insert gamma-matrix contracted field strengths $f_{j}$ of the bosons in the complement of $A$ adjacent to the four fermion wavefunctions.

To simplify the notation, let us define a "field-strength-inserted" fermion wave function, $\mathcal{X}_{i, B_{i}}$ for a fermion $i$ and a set of bosons $B_{i}=\left\{b_{1}, b_{2}, \ldots, b_{p}\right\}$,

$$
\mathcal{X}_{i, B_{i}}^{\alpha} \equiv \sum_{\omega \in S_{p}} \frac{\left(f_{\omega\left(b_{1}\right)} f_{\omega\left(b_{2}\right)} \cdots f_{\omega\left(b_{p}\right)}\right)^{\alpha}{ }_{\beta} \chi_{i}^{\beta}}{\sigma_{\omega\left(b_{1}\right), \omega\left(b_{2}\right)} \sigma_{\omega\left(b_{2}\right), \omega\left(b_{3}\right)} \cdots \sigma_{\omega\left(b_{p-1}\right), \omega\left(b_{p}\right)} \sigma_{\omega\left(b_{p}\right), i}},
$$

where we sum over permutations of $B_{i}$. In (B.21), we have one of the simplest examples

$$
\mathcal{X}_{i, b}=\frac{f_{b} \chi_{i}}{\sigma_{b, i}}=\frac{\chi_{i} f_{b}}{\sigma_{i, b}}, \quad \mathcal{X}_{i,\left\{b_{1}, b_{2}\right\}}=\frac{f_{b_{1}} f_{b_{2}} \chi_{i}}{\sigma_{b_{1}, b_{2}} \sigma_{b_{2}, i}}+\left(b_{1} \leftrightarrow b_{2}\right)=\frac{\chi_{i} f_{b_{2}} f_{b_{1}}}{\sigma_{i, b_{2}} \sigma_{b_{2}, b_{1}}}+\left(b_{1} \leftrightarrow b_{2}\right) .
$$

With this definition, the numerator $\left(\chi_{1} \gamma^{\mu} \chi_{2}\right)\left(\chi_{3} \gamma_{\mu} \chi_{4}\right)$ in (B.20) is generalized to $\left(\mathcal{X}_{1, B_{1}} \gamma^{\mu} \mathcal{X}_{2, B_{2}}\right) \quad \times\left(\mathcal{X}_{3, B_{3}} \gamma_{\mu} \mathcal{X}_{4, B_{4}}\right)$, which has four sets of field-strength insertions $B_{1}$, $B_{2}, B_{3}, B_{4}$, associated with fermions $1,2,3,4$, respectively. This is symmetric for bosons in each set $B_{i}(i=1,2,3,4)$, and by the gamma-matrix identity

$$
\left(\gamma^{\lambda \rho} \gamma_{\mu}\right)_{\alpha \beta} \gamma_{\gamma \delta}^{\mu}-\left(\gamma_{\mu} \gamma^{\lambda \rho}\right)_{\alpha \beta} \gamma_{\gamma \delta}^{\mu}+\gamma_{\alpha \beta}^{\mu}\left(\gamma^{\lambda \rho} \gamma_{\mu}\right)_{\gamma \delta}-\gamma_{\alpha \beta}^{\mu}\left(\gamma_{\mu} \gamma^{\lambda \rho}\right)_{\gamma \delta}=0
$$

underlying (B.22) has the correct $\mathrm{SL}_{2}$ weights for the $\sigma_{j}$ of all the bosons involved. Now it becomes clear how to write down the general form of the $n$-point correlator with four fermions:

$$
\begin{aligned}
& \left\langle\left(\prod_{i=1}^{4} V_{i}^{\left(-\frac{1}{2}\right)}\left(\sigma_{i}\right)\right)\left(\prod_{j=5}^{n} V_{j}^{(0)}\left(\sigma_{j}\right)\right)\right\rangle=\frac{1}{4} \sum_{\substack{\{5,6, \ldots, n\}=A \\
\cup B_{1} \cup \ldots \cup B_{4}}} \operatorname{Pf} \Psi_{A} \\
& \times\left(\frac{\left(\mathcal{X}_{1, B_{1}} \gamma^{\mu} \mathcal{X}_{2, B_{2}}\right)\left(\mathcal{X}_{3, B_{3}} \gamma_{\mu} \mathcal{X}_{4, B_{4}}\right)}{\sigma_{12} \sigma_{23} \sigma_{34} \sigma_{41}}+\frac{\left(\mathcal{X}_{1, B_{1}} \gamma^{\mu} \mathcal{X}_{3, B_{3}}\right)\left(\mathcal{X}_{2, B_{2}} \gamma_{\mu} \mathcal{X}_{4, B_{4}}\right)}{\sigma_{13} \sigma_{32} \sigma_{24} \sigma_{41}}\right) .
\end{aligned}
$$

It would be interesting to apply double-forward limits of this result to supersymmetric two-loop amplitudes [42].

\section{Details of gamma-matrix traces}

\section{C.1 Decomposition of $\operatorname{tr}_{S} \rightarrow \operatorname{tr}_{V}$}

Here we present a derivation of the decomposition of $\operatorname{tr}_{S}$ in terms of $\operatorname{tr}_{V}$ given in (3.11). First, we remind the reader of a well-known recursive formula for calculating $\gamma$ traces. 
Using that formula, we will find relative signs and the overall factor for the length- $n \operatorname{tr}_{\mathrm{V}}$ within the length- $n \operatorname{tr}_{S}$. Then we show how the multitrace terms arise from the recursive calculation of the $\gamma$ traces. For notational simplicity, we will focus on the sequential ordering of labels $\operatorname{tr}_{S}(1,2, \ldots, n)$ in $(3.4)$, with the understanding that other orderings can be reached by application of suitable permutations.

The parity-even piece of a generic-length $\gamma$ trace, in arbitrary dimension, can be computed using

$$
\left.\operatorname{tr}\left(\gamma_{\mu_{1}} \gamma_{\mu_{2}} \ldots \gamma_{\mu_{n}}\right)\right|_{\text {even }}=\left.\sum_{j=2}^{n}(-1)^{j} \eta_{\mu_{1} \mu_{j}} \operatorname{tr}\left(\gamma_{\mu_{2}} \ldots \tilde{\gamma}_{\mu_{j}} \ldots \gamma_{\mu_{n}}\right)\right|_{\text {even }}
$$

where $\operatorname{tr}\left(\gamma_{\mu_{2}} \ldots \tilde{\gamma}_{\mu_{j}} \ldots \gamma_{\mu_{n}}\right)$ is the trace of $n-2 \gamma \mathrm{s}$ with $\gamma_{\mu_{j}}$ removed. The recursion ends with $\operatorname{tr}\left(i d_{\mathrm{CA}}\right)$ which depends on the representation of the Clifford algebra, and therefore carries the $D$ dependence of the traces. We can use this formula to evaluate the $\operatorname{tr}_{S}$, using (2.12) to rewrite

$$
\operatorname{tr}_{\mathrm{S}}(1,2, \ldots, n)=\left.2^{-n} f_{1}^{\mu_{1} \nu_{1}} \ldots f_{n}^{\mu_{n} \nu_{n}} \operatorname{tr}\left(\gamma_{\mu_{1}} \gamma_{\nu_{1}} \ldots \gamma_{\mu_{n}} \gamma_{\nu_{n}}\right)\right|_{\text {even }}
$$

and noting that $\eta_{\mu \nu} f^{\mu \nu}=0$ means that all terms that generate $\eta_{\mu_{i} \nu_{i}}$ will not contribute to $\operatorname{tr}_{S}$. To see the patterns relevant to the $\operatorname{tr}_{\mathrm{V}}$ decomposition, we will need to make some clever use of the cyclic properties of tr. The maximal-length $\operatorname{tr}_{\mathrm{V}}$ terms can be written in terms of $\eta$ contractions as

$\frac{1}{2}\left(\operatorname{tr}_{\mathrm{V}}(1, \sigma(2), \ldots, \sigma(n))+(-1)^{n} \operatorname{tr}_{\mathrm{V}}(1, \sigma(n), \ldots, \sigma(2))\right)=f_{1}^{\mu_{1} \nu_{1}} \ldots f_{n}^{\mu_{n} \nu_{n}} \eta_{\nu_{1} \mu_{\sigma(2)}} \ldots \eta_{\nu_{\sigma(n)} \mu_{1}}$

with $\sigma \in S_{n-1}$, and the explicit reversal contribution is included to demonstrate the factor of $2^{-j}$ in (2.12). The $\eta_{\nu_{1} \mu_{\sigma(2)}}$ term can be directly sourced out of (C.1) by rotating the tr in (C.2) using cyclicity so that $\gamma_{\nu_{1}}$ is the first in the string. Then, since $\gamma_{\mu_{\sigma(2)}}$ will always occupy the even slots in the trace, this term always carries a + . The final $\eta_{\nu_{\sigma(n)} \mu_{1}}$ can always be chosen as the last step of the recursion (C.1), and thus also always carries a + . However, $\gamma_{\mu_{1}}$ remaining in the tr until the end is vitally important, as it is what breaks the symmetry between the two intermediate cases: $\sigma(i)$ coming before $\sigma(j)$ in $1,2, \ldots, n$, or coming after. If $\sigma(i)$ comes first, then the tr can be cycled such that $\gamma_{\nu_{\sigma(i)}}$ is at the front of the trace, and this cycling will never put $\gamma_{\mu_{1}}$ between $\gamma_{\nu_{\sigma(i)}}$ and $\gamma_{\mu_{\sigma(j)}}$. Since each pair of $\gamma_{\mu} \gamma_{\nu}$ can be removed in adjacent steps of this recursion, there will always be an even number of $\gamma$ between $\gamma_{\nu_{\sigma(i)}}$ and $\gamma_{\mu_{\sigma(j)}}$, and thus (C.1) will provide a + contribution. On the other hand, when $\sigma(i)$ comes after $\sigma(j)$, the process of cycling $\gamma_{\nu_{\sigma(i)}}$ to the front will always leave $\gamma_{\mu_{1}}$ between $\gamma_{\nu_{\sigma(i)}}$ and $\gamma_{\mu_{\sigma(j)}}$. As in the previous case, there will always be an even number of $\gamma \mathrm{s}$ removed between $\sigma(i)$ and $\sigma(j)$, but now $\gamma_{\mu_{1}}$ shifts the counting by 1 , so (C.1) will introduce a - sign. We collect all of the resulting signs into the ord function introduced in (3.12) to get

$$
\left.\operatorname{tr}_{\mathrm{S}}(1,2, \ldots, n)\right|_{\text {single }}=2^{D / 2-n-2} \sum_{\rho \in S_{n-1}} \operatorname{ord}_{\rho}^{i d} \operatorname{tr}_{\mathrm{V}}(1, \rho)
$$


which provides the leading trace term from (3.11). Notably, the reversed $\operatorname{tr}_{\mathrm{V}}$ from (C.3) is included as one of the elements of $\rho$.

The recursive realization of the $\operatorname{tr}(\gamma \ldots)$ in (C.1) also naturally generates the multiLorentz-trace terms. Each of the subtraces can be resolved, one at a time, in the same method as above. The $\gamma$ not participating in the targeted subtrace always cycle together, and thus only shift the counting between targeted $\gamma$ by an even number, never changing the sign. Each $\operatorname{tr}_{\mathrm{V}}$ picks up a factor of $\frac{1}{2}$ as in (C.3) to account for the reversal overcount, leading to the factor of $2^{-j}$ in (3.11).

\section{C.2 Higher $t_{2 n}$ tensors from $D=10$ SYM}

This appendix gives more details on the permutation symmetric tensors $t_{2 n}$ defined in (3.20). More specifically, we will determine the coefficients of $\operatorname{tr}_{\mathrm{V}}(1,2, \ldots, n)$ once the spinor traces are rewritten in terms of vectorial ones via (3.11). This will allow to verify the cancellation of the six-trace from the exceptionally simple expression (3.21) for $t_{12}$.

Using (3.11), we can count the + and - contributions of the longest $\operatorname{tr}_{\mathrm{V}}(1,2, \ldots, n)$ to the permutation sum (3.20) defining $t_{2 n}$. Since $t_{2 n}$ is fully permutation symmetric, it suffices to count the number of permutations in $S_{n-1}$ that generate a positive coefficient for $\operatorname{tr}_{\mathrm{V}}(1,2, \ldots, n)$ vs those that generate a negative one. These counts can be expressed directly in terms of the Eulerian numbers

$$
\left\langle\begin{array}{l}
i \\
k
\end{array}\right\rangle=\sum_{j=0}^{k+1}(-1)^{j}\left(\begin{array}{c}
i+1 \\
j
\end{array}\right)(k-j+1)^{i}
$$

which count the number of permutations of length $i$ that have $k$ permutation ascents; adjacent labels in the permutation $\rho$ that have $\rho_{j}<\rho_{j+1}$ are a permutation ascent. This is exactly the information needed by the $\operatorname{ord}_{\sigma}^{\rho} \operatorname{sign}(3.12)$, and as such those terms with $k$ even will carry a + sign, while $k$ odd will carry a.$-^{21}$ The symmetric tensor $t_{2 n}\left(f_{1}, f_{2}, \ldots, f_{n}\right)$ will contain the term $\operatorname{tr}_{\mathrm{V}}(1,2, \ldots, n)$ with a coefficient given by

$$
\operatorname{coef}\left(t_{2 n}\left(f_{1}, f_{2}, f_{3}, \ldots, f_{n}\right), \operatorname{tr}_{\mathrm{V}}(1,2, \ldots, n)\right)=\frac{2}{(n-1) !}\left[2-2^{3-n} \sum_{k=0}^{n-1}(-1)^{k}\left\langle\begin{array}{c}
n-1 \\
k
\end{array}\right\rangle\right] .
$$

The additional overall factor of 2 is due to the parity properties (3.5). As a necessary condition for the simplification (3.21) of $t_{12}$, the case with $n=6$ gives

$$
\begin{aligned}
\operatorname{coef}\left(t_{12}\left(f_{1}, f_{2}, \ldots, f_{6}\right), \operatorname{tr}_{\mathrm{V}}(1,2, \ldots, 6)\right) & =\frac{1}{60}\left[2-2^{-3} \sum_{k=0}^{5}(-1)^{k}\left\langle\begin{array}{l}
5 \\
k
\end{array}\right\rangle\right] \\
& =\frac{1}{60}\left[2-\frac{1}{8}(68-52)\right]=0,
\end{aligned}
$$

so there is no contribution of $\operatorname{tr}_{\mathrm{V}}(1,2, \ldots, 6)$ to correlators $(3.15)$ of $D=10$ SYM up to and including seven points. However, all other even $n$ admit length- $n$ Lorentz traces.

\footnotetext{
${ }^{21}$ The sign of ord is actually set by $i-k$, but since $i$ is even, $i-k$ and $k$ have the same parity.
} 


\section{C.3 Parity-odd traces}

In this appendix, we derive the parity-odd $\gamma$ trace expansion used in (5.12). Namely, our goal is to work out an evaluation of $\operatorname{tr}_{\text {odd }}\left(\gamma^{\mu_{1}} \ldots\right)$ defined by (5.4) in terms of $\varepsilon^{\mu_{i} \ldots}$. We start by making the standard identification of tensor structures ${ }^{22}$

$$
\operatorname{tr}_{\text {odd }}\left(\gamma^{\mu_{1}} \gamma^{\mu_{2}} \ldots \gamma^{\mu_{D}}\right)=i 2^{D / 2-1} \varepsilon^{\mu_{1} \ldots \mu_{D}}=\frac{i}{D !} \varepsilon^{\nu_{1} \ldots \nu_{D}}\left(\left.\operatorname{tr}\left(\gamma_{\nu_{1}} \ldots \gamma_{\nu_{D}} \gamma^{\mu_{1}} \ldots \gamma^{\mu_{D}}\right)\right|_{\text {even }}\right)
$$

which gives us the natural extension to more $\gamma$ in $\operatorname{tr}_{\text {odd }}(\ldots)$

$$
\operatorname{tr}_{\text {odd }}\left(\gamma^{\mu_{1}} \ldots \gamma^{\mu_{D+1}} \gamma^{\mu_{D+2}}\right)=\frac{i}{D !} \varepsilon^{\nu_{1} \ldots \nu_{D}}\left(\left.\operatorname{tr}\left(\gamma_{\nu_{1}} \ldots \gamma_{\nu_{D}} \gamma^{\mu_{1}} \ldots \gamma^{\mu_{D+1}} \gamma^{\mu_{D+2}}\right)\right|_{\text {even }}\right)
$$

From here, we could directly run the recursive evaluation from (C.1) on the right-hand side. However, it is worth pointing out an interesting feature of the calculation: the evaluation order will fully contract the $\varepsilon^{\nu \cdots}$ first, and then leave behind $\operatorname{tr}\left(\gamma^{\mu_{i}} \gamma^{\mu_{j}}\right)$ that are not contracted into the $\varepsilon$. Thus, running the recursive evaluation until the $\varepsilon$ is completely contracted, we find

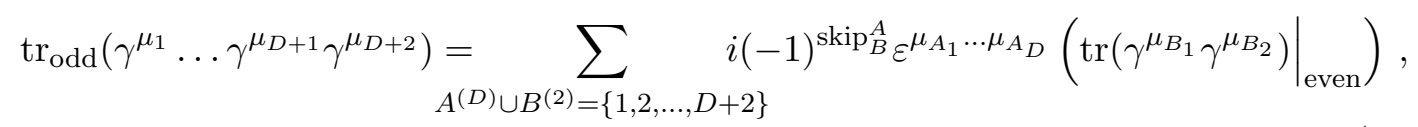

where the summation range $A^{(D)} \cup B^{(2)}=\{1,2, \ldots, D+2\}$ follows our convention of $A$ and $B$ being disjoint ordered subsets of $\{1,2, \ldots, D+2\}$, with the additional constraint that $A$ has length $D$, and $B$ length 2 . The sign $(-1)^{\text {skip }_{B}^{A}}$ compensates for skipping over the $\gamma^{\mu_{B_{i}}}$ as the $\gamma^{\mu_{A_{j}}}$ are paired with $\gamma_{\nu_{k}}$, ensuring that all of the terms in the remaining $B$ trace eventually have the correct relative signs. For the simple case in (C.10), $\operatorname{skip}_{B}^{A}$ is the number of $A_{i}$ between the two elements of $B$, which can in turn be reduced to the representation given in (5.12).

In order to generalize this computation to larger numbers $(D+2 j)$ of $\gamma \mathrm{s}$ with $j \geq 2$, we need to more carefully account for skip. As mentioned, it needs to restore the signs required in (C.1) that were dropped when separating the indices into the $A$ and $B$ set. A convenient definition for $\operatorname{skip}_{B}^{A}$ that accomplishes this is

$$
\operatorname{skip}_{B}^{A}=\sum_{i=1}^{D} \text { number of elements of } B \text { before } A_{i} .
$$

Note that this definition exactly captures the behavior described by (5.12): an even separation between the $B_{i}$ will have

$$
\operatorname{skip}_{B}^{A}=0+\cdots+\underbrace{1+\cdots+1}_{\text {even }}+2+\cdots \rightarrow(-1)^{\text {skip }_{B}^{A}}=1
$$

\footnotetext{
${ }^{22}$ This identification is based on the representation of the $2^{D / 2} \times 2^{D / 2}$ chirality matrix $\Gamma_{D+1}$ in terms of antisymmetrized products $\varepsilon^{\mu_{1} \mu_{2} \ldots \mu_{D}} \Gamma_{\mu_{1}} \Gamma_{\mu_{2}} \ldots \Gamma_{\mu_{D}}$ of Dirac gamma matrices $\Gamma_{\mu_{j}}$.
} 
whereas an odd separation will give

$$
\operatorname{skip}_{B}^{A}=0+\cdots+\underbrace{1+\cdots+1}_{\text {odd }}+2+\cdots \rightarrow(-1)^{\text {skip }_{B}^{A}}=-1 .
$$

All of these considerations allow us to generalize the computation fully

$$
\operatorname{tr}_{\text {odd }}\left(\gamma^{\mu_{1}} \ldots \gamma^{\mu_{D+2 j}}\right)=\sum_{\substack{A^{(D)} \cup B^{(2 j)} \\=\{1,2, \ldots, D+2 j\}}} i(-1)^{\operatorname{skip}_{B}^{A}} \varepsilon^{\mu_{A_{1}} \ldots \mu_{A_{D}}}\left(\left.\operatorname{tr}\left(\gamma^{\mu_{B_{1}}} \ldots \gamma^{\mu_{B_{2 j}}}\right)\right|_{\text {even }}\right) .
$$

Notably, this construction specifically includes (5.6) as the $j=0$ case using $\operatorname{tr}\left(i d_{\mathrm{CA}}\right)=$ $2^{D / 2-1}$.

Open Access. This article is distributed under the terms of the Creative Commons Attribution License (CC-BY 4.0), which permits any use, distribution and reproduction in any medium, provided the original author(s) and source are credited.

\section{References}

[1] Z. Bern, J.J.M. Carrasco and H. Johansson, New Relations for Gauge-Theory Amplitudes, Phys. Rev. D 78 (2008) 085011 [arXiv: 0805.3993] [inSPIRE].

[2] Z. Bern, J.J.M. Carrasco and H. Johansson, Perturbative Quantum Gravity as a Double Copy of Gauge Theory, Phys. Rev. Lett. 105 (2010) 061602 [arXiv: 1004.0476] [INSPIRE].

[3] Z. Bern, J.J. Carrasco, W.-M. Chen, H. Johansson and R. Roiban, Gravity Amplitudes as Generalized Double Copies of Gauge-Theory Amplitudes, Phys. Rev. Lett. 118 (2017) 181602 [arXiv: 1701.02519] [INSPIRE].

[4] Z. Bern, J.J. Carrasco, M. Chiodaroli, H. Johansson and R. Roiban, The Duality Between Color and Kinematics and its Applications, arXiv:1909.01358 [INSPIRE].

[5] H. Kawai, D.C. Lewellen and S.H.H. Tye, A Relation Between Tree Amplitudes of Closed and Open Strings, Nucl. Phys. B 269 (1986) 1 [inSPIRE].

[6] Z. Bern, T. Dennen, Y.-t. Huang and M. Kiermaier, Gravity as the Square of Gauge Theory, Phys. Rev. D 82 (2010) 065003 [arXiv: 1004.0693] [InSPIRE].

[7] Z. Bern, J.J.M. Carrasco, L.J. Dixon, H. Johansson and R. Roiban, Simplifying Multiloop Integrands and Ultraviolet Divergences of Gauge Theory and Gravity Amplitudes, Phys. Rev. D 85 (2012) 105014 [arXiv:1201.5366] [INSPIRE].

[8] Z. Bern, S. Davies, T. Dennen and Y.-t. Huang, Absence of Three-Loop Four-Point Divergences in N =4 Supergravity, Phys. Rev. Lett. 108 (2012) 201301 [arXiv:1202.3423] [INSPIRE].

[9] Z. Bern, S. Davies, T. Dennen, A.V. Smirnov and V.A. Smirnov, Ultraviolet Properties of $N=4$ Supergravity at Four Loops, Phys. Rev. Lett. 111 (2013) 231302 [arXiv:1309.2498] [INSPIRE].

[10] Z. Bern, S. Davies and T. Dennen, Enhanced ultraviolet cancellations in $\mathcal{N}=5$ supergravity at four loops, Phys. Rev. D 90 (2014) 105011 [arXiv: 1409.3089] [INSPIRE].

[11] Z. Bern, J.J.M. Carrasco, W.-M. Chen, H. Johansson, R. Roiban and M. Zeng, Five-loop four-point integrand of $N=8$ supergravity as a generalized double copy, Phys. Rev. D 96 (2017) 126012 [arXiv:1708. 06807] [INSPIRE]. 
[12] Z. Bern et al., Ultraviolet Properties of $\mathcal{N}=8$ Supergravity at Five Loops, Phys. Rev. D 98 (2018) 086021 [arXiv:1804.09311] [INSPIRE].

[13] C.R. Mafra, O. Schlotterer and S. Stieberger, Explicit BCJ Numerators from Pure Spinors, JHEP 07 (2011) 092 [arXiv: 1104.5224] [INSPIRE].

[14] C.R. Mafra, O. Schlotterer and S. Stieberger, Complete N-Point Superstring Disk Amplitude I. Pure Spinor Computation, Nucl. Phys. B 873 (2013) 419 [arXiv:1106.2645] [INSPIRE].

[15] C.R. Mafra and O. Schlotterer, Towards one-loop SYM amplitudes from the pure spinor BRST cohomology, Fortsch. Phys. 63 (2015) 105 [arXiv: 1410.0668] [INSPIRE].

[16] S. He, R. Monteiro and O. Schlotterer, String-inspired BCJ numerators for one-loop MHV amplitudes, JHEP 01 (2016) 171 [arXiv: 1507.06288] [INSPIRE].

[17] C.R. Mafra and O. Schlotterer, Two-loop five-point amplitudes of super Yang-Mills and supergravity in pure spinor superspace, JHEP 10 (2015) 124 [arXiv:1505.02746] [INSPIRE].

[18] N.E.J. Bjerrum-Bohr, P.H. Damgaard and P. Vanhove, Minimal Basis for Gauge Theory Amplitudes, Phys. Rev. Lett. 103 (2009) 161602 [arXiv:0907.1425] [INSPIRE].

[19] S. Stieberger, Open \& Closed vs. Pure Open String Disk Amplitudes, arXiv:0907.2211 [INSPIRE].

[20] P. Tourkine and P. Vanhove, Higher-loop amplitude monodromy relations in string and gauge theory, Phys. Rev. Lett. 117 (2016) 211601 [arXiv:1608.01665] [INSPIRE].

[21] S. Hohenegger and S. Stieberger, Monodromy Relations in Higher-Loop String Amplitudes, Nucl. Phys. B 925 (2017) 63 [arXiv:1702.04963] [INSPIRE].

[22] A. Ochirov, P. Tourkine and P. Vanhove, One-loop monodromy relations on single cuts, JHEP 10 (2017) 105 [arXiv:1707.05775] [INSPIRE].

[23] P. Tourkine, Integrands and loop momentum in string and field theory, Phys. Rev. D 102 (2020) 026006 [arXiv: 1901.02432] [INSPIRE].

[24] E. Casali, S. Mizera and P. Tourkine, Monodromy relations from twisted homology, JHEP 12 (2019) 087 [arXiv: 1910.08514] [INSPIRE].

[25] F. Cachazo, S. He and E.Y. Yuan, Scattering of Massless Particles in Arbitrary Dimensions, Phys. Rev. Lett. 113 (2014) 171601 [arXiv:1307.2199] [INSPIRE].

[26] F. Cachazo, S. He and E.Y. Yuan, Scattering of Massless Particles: Scalars, Gluons and Gravitons, JHEP 07 (2014) 033 [arXiv:1309.0885] [INSPIRE].

[27] F. Cachazo, S. He and E.Y. Yuan, Scattering equations and Kawai-Lewellen-Tye orthogonality, Phys. Rev. D 90 (2014) 065001 [arXiv:1306.6575] [INSPIRE].

[28] L. Mason and D. Skinner, Ambitwistor strings and the scattering equations, JHEP 07 (2014) 048 [arXiv: 1311.2564] [INSPIRE].

[29] N. Berkovits, Infinite Tension Limit of the Pure Spinor Superstring, JHEP 03 (2014) 017 [arXiv:1311.4156] [INSPIRE].

[30] T. Adamo, E. Casali and D. Skinner, Ambitwistor strings and the scattering equations at one loop, JHEP 04 (2014) 104 [arXiv: 1312.3828] [INSPIRE].

[31] T. Adamo and E. Casali, Scattering equations, supergravity integrands, and pure spinors, JHEP 05 (2015) 120 [arXiv: 1502.06826] [INSPIRE]. 
[32] E. Casali, Y. Geyer, L. Mason, R. Monteiro and K.A. Roehrig, New Ambitwistor String Theories, JHEP 11 (2015) 038 [arXiv: 1506.08771] [InSPIRE].

[33] F. Cachazo, S. He and E.Y. Yuan, Scattering Equations and Matrices: From Einstein To Yang-Mills, DBI and NLSM, JHEP 07 (2015) 149 [arXiv:1412.3479] [INSPIRE].

[34] M. Chiodaroli, M. Günaydin, H. Johansson and R. Roiban, Scattering amplitudes in $\mathcal{N}=2$ Maxwell-Einstein and Yang-Mills/Einstein supergravity, JHEP 01 (2015) 081 [arXiv: 1408.0764] [INSPIRE].

[35] M. Chiodaroli, M. Günaydin, H. Johansson and R. Roiban, Explicit Formulae for Yang-Mills-Einstein Amplitudes from the Double Copy, JHEP 07 (2017) 002 [arXiv: 1703.00421] [INSPIRE].

[36] Y. Geyer, L. Mason, R. Monteiro and P. Tourkine, Loop Integrands for Scattering Amplitudes from the Riemann Sphere, Phys. Rev. Lett. 115 (2015) 121603 [arXiv: 1507.00321] [INSPIRE].

[37] Y. Geyer, L. Mason, R. Monteiro and P. Tourkine, One-loop amplitudes on the Riemann sphere, JHEP 03 (2016) 114 [arXiv:1511.06315] [INSPIRE].

[38] F. Cachazo, S. He and E.Y. Yuan, One-Loop Corrections from Higher Dimensional Tree Amplitudes, JHEP 08 (2016) 008 [arXiv:1512.05001] [INSPIRE].

[39] Y. Geyer, L. Mason, R. Monteiro and P. Tourkine, Two-Loop Scattering Amplitudes from the Riemann Sphere, Phys. Rev. D 94 (2016) 125029 [arXiv:1607.08887] [InSPIRE].

[40] Y. Geyer and R. Monteiro, Gluons and gravitons at one loop from ambitwistor strings, JHEP 03 (2018) 068 [arXiv:1711.09923] [INSPIRE].

[41] Y. Geyer and R. Monteiro, Two-Loop Scattering Amplitudes from Ambitwistor Strings: from Genus Two to the Nodal Riemann Sphere, JHEP 11 (2018) 008 [arXiv:1805.05344] [INSPIRE].

[42] Y. Geyer, R. Monteiro and R. Stark-Muchão, Two-Loop Scattering Amplitudes: Double-Forward Limit and Colour-Kinematics Duality, JHEP 12 (2019) 049 [arXiv: 1908.05221] [INSPIRE].

[43] S. He and O. Schlotterer, New Relations for Gauge-Theory and Gravity Amplitudes at Loop Level, Phys. Rev. Lett. 118 (2017) 161601 [arXiv:1612.00417] [INSPIRE].

[44] S. He, O. Schlotterer and Y. Zhang, New BCJ representations for one-loop amplitudes in gauge theories and gravity, Nucl. Phys. B 930 (2018) 328 [arXiv:1706.00640] [InSPIRE].

[45] S. He and E.Y. Yuan, One-loop Scattering Equations and Amplitudes from Forward Limit, Phys. Rev. D 92 (2015) 105004 [arXiv:1508.06027] [INSPIRE].

[46] C. Cardona and H. Gomez, Elliptic scattering equations, JHEP 06 (2016) 094 [arXiv: 1605.01446] [INSPIRE].

[47] C. Cardona and H. Gomez, CHY-Graphs on a Torus, JHEP 10 (2016) 116 [arXiv: 1607.01871] [INSPIRE].

[48] H. Gomez, $\Lambda$ scattering equations, JHEP 06 (2016) 101 [arXiv:1604.05373] [INSPIRE].

[49] H. Gomez, S. Mizera and G. Zhang, CHY Loop Integrands from Holomorphic Forms, JHEP 03 (2017) 092 [arXiv: 1612.06854] [INSPIRE].

[50] H. Gomez, Quadratic Feynman Loop Integrands From Massless Scattering Equations, Phys. Rev. D 95 (2017) 106006 [arXiv: 1703.04714] [INSPIRE]. 
[51] H. Gomez, C. Lopez-Arcos and P. Talavera, One-loop Parke-Taylor factors for quadratic propagators from massless scattering equations, JHEP 10 (2017) 175 [arXiv:1707.08584] [INSPIRE].

[52] N. Ahmadiniaz, H. Gomez and C. Lopez-Arcos, Non-planar one-loop Parke-Taylor factors in the CHY approach for quadratic propagators, JHEP 05 (2018) 055 [arXiv:1802.00015] [INSPIRE].

[53] J. Agerskov, N.E.J. Bjerrum-Bohr, H. Gomez and C. Lopez-Arcos, One-Loop Yang-Mills Integrands from Scattering Equations, Phys. Rev. D 102 (2020) 045023 [arXiv:1910.03602] [INSPIRE].

[54] A. Tsuchiya, More on One Loop Massless Amplitudes of Superstring Theories, Phys. Rev. D 39 (1989) 1626 [INSPIRE].

[55] S. Stieberger and T.R. Taylor, NonAbelian Born-Infeld action and type 1. - heterotic duality 2: Nonrenormalization theorems, Nucl. Phys. B 648 (2003) 3 [hep-th/0209064] [InSPIRE].

[56] M. Bianchi and A.V. Santini, String predictions for near future colliders from one-loop scattering amplitudes around D-brane worlds, JHEP 12 (2006) 010 [hep-th/0607224] [INSPIRE].

[57] J. Broedel, C.R. Mafra, N. Matthes and O. Schlotterer, Elliptic multiple zeta values and one-loop superstring amplitudes, JHEP 07 (2015) 112 [arXiv:1412.5535] [INSPIRE].

[58] M. Berg, I. Buchberger and O. Schlotterer, From maximal to minimal supersymmetry in string loop amplitudes, JHEP 04 (2017) 163 [arXiv: 1603.05262] [INSPIRE].

[59] H. Gomez and E.Y. Yuan, N-point tree-level scattering amplitude in the new Berkovits" string, JHEP 04 (2014) 046 [arXiv:1312.5485] [INSPIRE].

[60] S. Lee, C.R. Mafra and O. Schlotterer, Non-linear gauge transformations in D $=10 S Y M$ theory and the BCJ duality, JHEP 03 (2016) 090 [arXiv:1510.08843] [INSPIRE].

[61] C.R. Mafra and O. Schlotterer, Berends-Giele recursions and the BCJ duality in superspace and components, JHEP 03 (2016) 097 [arXiv: 1510.08846] [INSPIRE].

[62] C.R. Mafra and O. Schlotterer, One-loop superstring six-point amplitudes and anomalies in pure spinor superspace, JHEP 04 (2016) 148 [arXiv: 1603.04790] [INSPIRE].

[63] C.R. Mafra and O. Schlotterer, Towards the n-point one-loop superstring amplitude. Part I. Pure spinors and superfield kinematics, JHEP 08 (2019) 090 [arXiv:1812.10969] [INSPIRE].

[64] C.R. Mafra and O. Schlotterer, Towards the n-point one-loop superstring amplitude. Part III. One-loop correlators and their double-copy structure, JHEP 08 (2019) 092 [arXiv: 1812.10971] [INSPIRE].

[65] K.A. Roehrig and D. Skinner, A Gluing Operator for the Ambitwistor String, JHEP 01 (2018) 069 [arXiv:1709.03262] [InSPIRE].

[66] C.-H. Fu, Y.-J. Du, R. Huang and B. Feng, Expansion of Einstein-Yang-Mills Amplitude, JHEP 09 (2017) 021 [arXiv:1702.08158] [INSPIRE].

[67] C. Cardona, B. Feng, H. Gomez and R. Huang, Cross-ratio Identities and Higher-order Poles of CHY-integrand, JHEP 09 (2016) 133 [arXiv:1606.00670] [INSPIRE].

[68] D. Nandan, J. Plefka, O. Schlotterer and C. Wen, Einstein-Yang-Mills from pure Yang-Mills amplitudes, JHEP 10 (2016) 070 [arXiv: 1607.05701] [INSPIRE]. 
[69] N.E.J. Bjerrum-Bohr, J.L. Bourjaily, P.H. Damgaard and B. Feng, Manifesting Color-Kinematics Duality in the Scattering Equation Formalism, JHEP 09 (2016) 094 [arXiv: 1608.00006] [INSPIRE].

[70] O. Schlotterer, Amplitude relations in heterotic string theory and Einstein-Yang-Mills, JHEP 11 (2016) 074 [arXiv:1608.00130] [INSPIRE].

[71] F. Teng and B. Feng, Expanding Einstein-Yang-Mills by Yang-Mills in CHY frame, JHEP 05 (2017) 075 [arXiv: 1703.01269] [INSPIRE].

[72] A. Edison and F. Teng, Efficient Calculation of Crossing Symmetric BCJ Tree Numerators, arXiv:2005.03638 [INSPIRE].

[73] C.R. Mafra and O. Schlotterer, Multiparticle SYM equations of motion and pure spinor BRST blocks, JHEP 07 (2014) 153 [arXiv:1404.4986] [INSPIRE].

[74] F.A. Berends and W.T. Giele, Recursive Calculations for Processes with n Gluons, Nucl. Phys. B 306 (1988) 759 [INSPIRE].

[75] E. Bridges and C.R. Mafra, Algorithmic construction of SYM multiparticle superfields in the BCJ gauge, JHEP 10 (2019) 022 [arXiv:1906.12252] [INSPIRE].

[76] F. Cachazo, P. Cha and S. Mizera, Extensions of Theories from Soft Limits, JHEP 06 (2016) 170 [arXiv: 1604.03893] [INSPIRE].

[77] P. Ramond, Dual Theory for Free Fermions, Phys. Rev. D 3 (1971) 2415 [InSPIRE].

[78] A. Neveu and J.H. Schwarz, Factorizable dual model of pions, Nucl. Phys. B 31 (1971) 86 [INSPIRE].

[79] E. D'Hoker and D.H. Phong, The Geometry of String Perturbation Theory, Rev. Mod. Phys. 60 (1988) 917 [INSPIRE].

[80] D. Friedan, S.H. Shenker and E.J. Martinec, Covariant Quantization of Superstrings, Phys. Lett. B 160 (1985) 55 [INSPIRE].

[81] D. Friedan, E.J. Martinec and S.H. Shenker, Conformal Invariance, Supersymmetry and String Theory, Nucl. Phys. B 271 (1986) 93 [InSPIRE].

[82] V.G. Knizhnik, Covariant Fermionic Vertex in Superstrings, Phys. Lett. B 160 (1985) 403 [INSPIRE].

[83] J. Cohn, D. Friedan, Z.-a. Qiu and S.H. Shenker, Covariant Quantization of Supersymmetric String Theories: The Spinor Field of the Ramond-Neveu-Schwarz Model, Nucl. Phys. B 278 (1986) 577 [INSPIRE].

[84] C.S. Lam and Y.-P. Yao, Evaluation of the Cachazo-He-Yuan gauge amplitude, Phys. Rev. D 93 (2016) 105008 [arXiv: 1602.06419] [INSPIRE].

[85] V. Kostelecky, O. Lechtenfeld, W. Lerche, S. Samuel and S. Watamura, Conformal Techniques, Bosonization and Tree Level String Amplitudes, Nucl. Phys. B 288 (1987) 173 [INSPIRE].

[86] H. Frost, New directions for the ambitwistor string, Transfer Thesis, University of Oxford (2017), https://people.maths.ox.ac.uk/lmason/Theses/HF-Transfer.pdf.

[87] J.J. Atick and A. Sen, Covariant One Loop Fermion Emission Amplitudes in Closed String Theories, Nucl. Phys. B 293 (1987) 317 [InSPIRE]. 
[88] V. Kostelecky, O. Lechtenfeld, S. Samuel, D. Verstegen, S. Watamura and D. Sahdev, The Six Fermion Amplitude in the Superstring, Phys. Lett. B 183 (1987) 299 [INSPIRE].

[89] S. Lee and O. Schlotterer, Fermionic one-loop amplitudes of the RNS superstring, JHEP 03 (2018) 190 [arXiv:1710.07353] [INSPIRE].

[90] N. Berkovits, Super Poincaré covariant quantization of the superstring, JHEP 04 (2000) 018 [hep-th/0001035] [INSPIRE].

[91] M.B. Green, J.H. Schwarz and L. Brink, $N=4$ Yang-Mills and $N=8$ Supergravity as Limits of String Theories, Nucl. Phys. B 198 (1982) 474 [INSPIRE].

[92] T. van Ritbergen, A.N. Schellekens and J.A.M. Vermaseren, Group theory factors for Feynman diagrams, Int. J. Mod. Phys. A 14 (1999) 41 [hep-ph/9802376] [INSPIRE].

[93] F. Cachazo, S. He and E.Y. Yuan, Einstein-Yang-Mills Scattering Amplitudes From Scattering Equations, JHEP 01 (2015) 121 [arXiv:1409.8256] [INSPIRE].

[94] S. He, F. Teng and Y. Zhang, String amplitudes from field-theory amplitudes and vice versa, Phys. Rev. Lett. 122 (2019) 211603 [arXiv:1812.03369] [INSPIRE].

[95] S. He, F. Teng and Y. Zhang, String Correlators: Recursive Expansion, Integration-by-Parts and Scattering Equations, JHEP 09 (2019) 085 [arXiv: 1907.06041] [INSPIRE].

[96] Z. Bern, L.J. Dixon, D.C. Dunbar and D.A. Kosower, One loop n point gauge theory amplitudes, unitarity and collinear limits, Nucl. Phys. B 425 (1994) 217 [hep-ph/9403226] [INSPIRE].

[97] Z. Bern and A.G. Morgan, Supersymmetry relations between contributions to one loop gauge boson amplitudes, Phys. Rev. D 49 (1994) 6155 [hep-ph/9312218] [INSPIRE].

[98] Z. Bern, String based perturbative methods for gauge theories, in Theoretical Advanced Study Institute (TASI 92): From Black Holes and Strings to Particles, pp. 0471-536, 6, 1992, hep-ph/9304249 [inSPIRE].

[99] Z. Bern, S. Davies, T. Dennen, Y.-t. Huang and J. Nohle, Color-Kinematics Duality for Pure Yang-Mills and Gravity at One and Two Loops, Phys. Rev. D 92 (2015) 045041 [arXiv: 1303.6605] [INSPIRE].

[100] L. Clavelli, P.H. Cox and B. Harms, Parity Violating One Loop Six Point Function in Type I Superstring Theory, Phys. Rev. D 35 (1987) 1908 [InSPIRE].

[101] D.J. Gross and P.F. Mende, Modular Subgroups, Odd Spin Structures and Gauge Invariance in the Heterotic String, Nucl. Phys. B 291 (1987) 653 [InSPIRE].

[102] P.H. Frampton and T.W. Kephart, Explicit Evaluation of Anomalies in Higher Dimensions, Phys. Rev. Lett. 50 (1983) 1343 [Erratum ibid. 51 (1983) 232] [INSPIRE].

[103] P.H. Frampton and T.W. Kephart, The Analysis of Anomalies in Higher Space-time Dimensions, Phys. Rev. D 28 (1983) 1010 [InSPIRE].

[104] B. Zumino, Y.-S. Wu and A. Zee, Chiral Anomalies, Higher Dimensions, and Differential Geometry, Nucl. Phys. B 239 (1984) 477 [InSPIRE].

[105] M.B. Green, C.R. Mafra and O. Schlotterer, Multiparticle one-loop amplitudes and S-duality in closed superstring theory, JHEP 10 (2013) 188 [arXiv:1307.3534] [INSPIRE].

[106] M. Berg, I. Buchberger and O. Schlotterer, String-motivated one-loop amplitudes in gauge theories with half-maximal supersymmetry, JHEP 07 (2017) 138 [arXiv:1611.03459] [INSPIRE]. 
[107] W.-M. Chen, Y.-t. Huang and D.A. McGady, Anomalies without an action, arXiv: 1402.7062 [INSPIRE].

[108] H. Gomez and C.R. Mafra, The closed-string 3-loop amplitude and S-duality, JHEP 10 (2013) 217 [arXiv: 1308.6567] [INSPIRE].

[109] L.M. Garozzo, L. Queimada and O. Schlotterer, Berends-Giele currents in Bern-Carrasco-Johansson gauge for $F^{3}$ - and $F^{4}$-deformed Yang-Mills amplitudes, JHEP 02 (2019) 078 [arXiv: 1809.08103] [INSPIRE].

[110] C.R. Mafra and O. Schlotterer, Cohomology foundations of one-loop amplitudes in pure spinor superspace, arXiv:1408.3605 [INSPIRE].

[111] J.A. Minahan, One Loop Amplitudes on Orbifolds and the Renormalization of Coupling Constants, Nucl. Phys. B 298 (1988) 36 [InSPIRE].

[112] C. Baadsgaard, N.E.J. Bjerrum-Bohr, J.L. Bourjaily, S. Caron-Huot, P.H. Damgaard and B. Feng, New Representations of the Perturbative S-matrix, Phys. Rev. Lett. 116 (2016) 061601 [arXiv: 1509.02169] [INSPIRE].

[113] D. Haertl and O. Schlotterer, Higher Loop Spin Field Correlators in Various Dimensions, Nucl. Phys. B 849 (2011) 364 [arXiv:1011.1249] [InSPIRE]. 